\title{
ON HOMOGENIZATION OF THE FIRST INITIAL-BOUNDARY VALUE PROBLEM FOR PERIODIC HYPERBOLIC SYSTEMS
}

\author{
YU. M. MESHKOVA
}

\begin{abstract}
Let $\mathcal{O} \subset \mathbb{R}^{d}$ be a bounded domain of class $C^{3,1}$. In $L_{2}\left(\mathcal{O} ; \mathbb{C}^{n}\right)$, we consider a self-adjoint matrix strongly elliptic second order differential operator $B_{D, \varepsilon}, 0<\varepsilon \leqslant 1$, with the Dirichlet boundary condition. The coefficients of the operator $B_{D, \varepsilon}$ are periodic and depend on $\mathbf{x} / \varepsilon$. We are interested in the behavior of the operators $\cos \left(t B_{D, \varepsilon}^{1 / 2}\right)$ and $B_{D, \varepsilon}^{-1 / 2} \sin \left(t B_{D, \varepsilon}^{1 / 2}\right), t \in \mathbb{R}$, in the small period limit. For these operators, approximations in the norm of operators acting from a certain subspace $\mathcal{H}$ of the Sobolev space $H^{4}\left(\mathcal{O} ; \mathbb{C}^{n}\right)$ to $L_{2}\left(\mathcal{O} ; \mathbb{C}^{n}\right)$ are found. Moreover, for $B_{D, \varepsilon}^{-1 / 2} \sin \left(t B_{D, \varepsilon}^{1 / 2}\right)$, the approximation with the corrector in the norm of operators acting from $\mathcal{H} \subset H^{4}\left(\mathcal{O} ; \mathbb{C}^{n}\right)$ to $H^{1}\left(\mathcal{O} ; \mathbb{C}^{n}\right)$ is obtained. The results are applied to homogenization for the solution of the first initial-boundary value problem for the hyperbolic equation $\partial_{t}^{2} \mathbf{u}_{\varepsilon}=-B_{D, \varepsilon} \mathbf{u}_{\varepsilon}$.
\end{abstract}

\section{Contents}

Introduction

1. Homogenization results for the elliptic Dirichlet problem

2. Problem setting. Main results

3. Proof of Theorems 2.1 and 2.2

References

\section{INTRODUCTION}

The paper concerns homogenization theory of periodic differential operators (DO's). A broad literature is devoted to homogenization theory, see, e. g., the books BaPa, BeLPap, ZhKO, Sa].

0.1. Problem setting. Let $\Gamma \subset \mathbb{R}^{d}$ be a lattice and let $\Omega$ be the cell of the lattice $\Gamma$. For $\Gamma$-periodic functions in $\mathbb{R}^{d}$, we use the following notation $f^{\varepsilon}(\mathbf{x}):=f\left(\varepsilon^{-1} \mathbf{x}\right), \varepsilon>0$. Let $\mathcal{O} \subset \mathbb{R}^{d}$ be a bounded domain of class $C^{1,1}$. In $L_{2}\left(\mathcal{O} ; \mathbb{C}^{n}\right)$, we consider a self-adjoint matrix strongly elliptic second order differential operator $B_{D, \varepsilon}, 0<\varepsilon \leqslant 1$, with the Dirichlet boundary condition. The principal part of the operator $B_{D, \varepsilon}$ is given in a factorized form $A_{\varepsilon}=b(\mathbf{D})^{*} g^{\varepsilon}(\mathbf{x}) b(\mathbf{D})$, where $b(\mathbf{D})=\sum_{l=1}^{d} b_{l} D_{l}$ is a matrix first order DO and $g(\mathbf{x})$ is a $\Gamma$-periodic matrix-valued function in $\mathbb{R}^{d}$ such that $g, g^{-1} \in L_{\infty}$ and $g(\mathbf{x})>0$. (The precise assumptions on $b(\mathbf{D})$ and $g$ are given below in Subsec. 1.3.) The operator $B_{D, \varepsilon}$ is given by the differential expression

$$
B_{\varepsilon}=b(\mathbf{D})^{*} g^{\varepsilon}(\mathbf{x}) b(\mathbf{D})+\sum_{j=1}^{d}\left(a_{j}^{\varepsilon}(\mathbf{x}) D_{j}+D_{j} a_{j}^{\varepsilon}(\mathbf{x})^{*}\right)+Q^{\varepsilon}(\mathbf{x})+\lambda I
$$

with the Dirichlet condition on $\partial \mathcal{O}$. Here $\Gamma$-periodic matrix-valued functions $a_{j}$, $j=1, \ldots, d$, and $Q$ belong to suitable $L_{p}(\Omega)$-spaces and the matrix $Q(\mathbf{x})$ is assumed to

2010 Mathematics Subject Classification. Primary 35B27. Secondary 35L53.

Key words and phrases. Periodic differential operators, hyperbolic systems, homogenization, operator error estimates.

Research is supported by the Russian Science Foundation grant no. 14-21-00035. 
be Hermitian. The constant $\lambda$ is chosen so that the operator $B_{D, \varepsilon}$ is positive definite. (The explicit assumptions on the coefficients are given below in Subsec. 1.4.) The precise definition of the operator $B_{D, \varepsilon}$ is given via the corresponding quadratic form on the Sobolev class $H_{0}^{1}\left(\mathcal{O} ; \mathbb{C}^{n}\right)$.

The coefficients of the operator $B_{D, \varepsilon}$ oscillate rapidly for small $\varepsilon$. We are interested in the behavior of the solution of the following problem for small $\varepsilon$ :

$$
\left\{\begin{array}{l}
\frac{\partial^{2} \mathbf{u}_{\varepsilon}}{\partial t^{2}}(\mathbf{x}, t)=-\left(B_{\varepsilon} \mathbf{u}_{\varepsilon}\right)(\mathbf{x}, t)+\mathbf{F}(\mathbf{x}, t),\left.\quad \mathbf{u}_{\varepsilon}(\cdot, t)\right|_{\partial \mathcal{O}}=0 \\
\mathbf{u}_{\varepsilon}(\mathbf{x}, 0)=\boldsymbol{\varphi}(\mathbf{x}), \quad \frac{\partial \mathbf{u}_{\varepsilon}}{\partial t}(\mathbf{x}, 0)=\boldsymbol{\psi}(\mathbf{x})
\end{array}\right.
$$

For $\boldsymbol{\varphi} \in H_{0}^{1}\left(\mathcal{O} ; \mathbb{C}^{n}\right), \boldsymbol{\psi} \in L_{2}\left(\mathcal{O} ; \mathbb{C}^{n}\right)$, and $\mathbf{F} \in L_{1, \text { loc }}\left(\mathbb{R} ; L_{2}\left(\mathcal{O} ; \mathbb{C}^{n}\right)\right)$, we have

$$
\mathbf{u}_{\varepsilon}(\cdot, t)=\cos \left(t B_{D, \varepsilon}^{1 / 2}\right) \boldsymbol{\varphi}+B_{D, \varepsilon}^{-1 / 2} \sin \left(t B_{D, \varepsilon}^{1 / 2}\right) \boldsymbol{\psi}+\int_{0}^{t} B_{D, \varepsilon}^{-1 / 2} \sin \left((t-\widetilde{t}) B_{D, \varepsilon}^{1 / 2}\right) \mathbf{F}(\cdot, \widetilde{t}) d \widetilde{t} .
$$

Thus, the question about the behavior of the solution of problem (0.2) is reduced to suitable approximations of the operators $\cos \left(t B_{D, \varepsilon}^{1 / 2}\right)$ and $B_{D, \varepsilon}^{-1 / 2} \sin \left(t B_{D, \varepsilon}^{1 / 2}\right)$ for small $\varepsilon$.

0.2. Main results. Our first main results are:

$$
\begin{aligned}
& \left\|\left(\cos \left(t B_{D, \varepsilon}^{1 / 2}\right)-\cos \left(t\left(B_{D}^{0}\right)^{1 / 2}\right)\right)\left(B_{D}^{0}\right)^{-2}\right\|_{L_{2}(\mathcal{O}) \rightarrow L_{2}(\mathcal{O})} \leqslant C \varepsilon\left(1+|t|^{5}\right), \\
& \left\|\left(B_{D, \varepsilon}^{-1 / 2} \sin \left(t B_{D, \varepsilon}^{1 / 2}\right)-\left(B_{D}^{0}\right)^{-1 / 2} \sin \left(t\left(B_{D}^{0}\right)^{1 / 2}\right)\right)\left(B_{D}^{0}\right)^{-2}\right\|_{L_{2}(\mathcal{O}) \rightarrow L_{2}(\mathcal{O})} \leqslant C \varepsilon|t|\left(1+|t|^{5}\right) .
\end{aligned}
$$

These estimates are valid for $t \in \mathbb{R}$ and sufficiently small $\varepsilon$. Here $B_{D}^{0}$ is the effective operator with constant coefficients. The second result is the following approximation

$$
\begin{aligned}
& \left\|\left(B_{D, \varepsilon}^{-1 / 2} \sin \left(t B_{D, \varepsilon}^{1 / 2}\right)-\left(B_{D}^{0}\right)^{-1 / 2} \sin \left(t\left(B_{D}^{0}\right)^{1 / 2}\right)-\varepsilon \mathcal{K}_{D}(\varepsilon ; t)\right)\left(B_{D}^{0}\right)^{-2}\right\|_{L_{2}(\mathcal{O}) \rightarrow H^{1}(\mathcal{O})} \\
& \quad \leqslant C \varepsilon^{1 / 2}\left(1+t^{6}\right) .
\end{aligned}
$$

Here $\mathcal{K}_{D}(\varepsilon ; t)$ is the corrector. It contains rapidly oscillating factors and so depends on $\varepsilon$. In the general case, the corrector contains a smoothing operator. For $d \leqslant 8$, if the boundary of the domain is sufficiently smooth, one can remove the smoothing operator from the corrector. The constants in estimates (0.3) -(0.5) can be controlled explicitly in terms of the problem data. The results of such type are called operator error estimates in homogenization theory. For $t$ fixed, estimates (0.3) and (0.4) are of the sharp order $O(\varepsilon)$. The order $O\left(\varepsilon^{1 / 2}\right)$ of estimate (0.5) is worse because of the boundary influence. It is impossible to obtain an analogue of estimate (0.5) for the operator $\cos \left(t B_{D, \varepsilon}^{1 / 2}\right)$. But the ,smoothed" cosine operator can be approximated:

$$
\begin{aligned}
& \left\|\left(\cos \left(t B_{D, \varepsilon}^{1 / 2}\right) B_{D, \varepsilon}^{-1}-\cos \left(t\left(B_{D}^{0}\right)^{1 / 2}\right)\left(B_{D}^{0}\right)^{-1}-\varepsilon \mathscr{K}_{D}(\varepsilon ; t)\right)\left(B_{D}^{0}\right)^{-1}\right\|_{L_{2}(\mathcal{O}) \rightarrow H^{1}(\mathcal{O})} \\
& \quad \leqslant C \varepsilon^{1 / 2}\left(1+|t|^{5}\right) .
\end{aligned}
$$

It is in accordance with the results of [BrOtFMu, see discussion in Subsec. 0.3 below.

0.3. Survey. At present, operator error estimates attract a great deal of attention. Interest to this topic was caused by the paper [BSu1] of M. Sh. Birman and T. A. Suslina. In [BSu1], the operator $A_{\varepsilon}=b(\mathbf{D})^{*} g^{\varepsilon}(\mathbf{x}) b(\mathbf{D})$ acting in $L_{2}\left(\mathbb{R}^{d} ; \mathbb{C}^{n}\right)$ was considered. By means of the spectral approach it was obtained that

$$
\left\|\left(A_{\varepsilon}+I\right)^{-1}-\left(A^{0}+I\right)^{-1}\right\|_{L_{2}\left(\mathbb{R}^{d}\right) \rightarrow L_{2}\left(\mathbb{R}^{d}\right)} \leqslant C \varepsilon .
$$

Here $A^{0}=b(\mathbf{D})^{*} g^{0} b(\mathbf{D})$ is the effective operator and $g^{0}$ is the constant effective matrix. In [BSu3], the operator $\left(A_{\varepsilon}+I\right)^{-1}$ was approximated in the $\left(L_{2} \rightarrow H^{1}\right)$-operator norm:

$$
\left\|\left(A_{\varepsilon}+I\right)^{-1}-\left(A^{0}+I\right)^{-1}-\varepsilon K(\varepsilon)\right\|_{L_{2}\left(\mathbb{R}^{d}\right) \rightarrow H^{1}\left(\mathbb{R}^{d}\right)} \leqslant C \varepsilon .
$$


The estimates (0.7) and (10.8) were later generalized to the operator $B_{\varepsilon}$ of the form (10.1) by T. A. Suslina [Su3].

Another approach to operator error estimates in homogenization theory was suggested by V. V. Zhikov [Zh]. In [Zh, ZhPas1], estimates of the form (0.7), (0.8) were obtained for the acoustics operator and for the elasticity operator. The „modified method of the first order approximation" or the "shift method", in the terminology of the authors, was based on the analysis of the first order approximation to the solution and introducing the additional parameter. Besides the problems in $\mathbb{R}^{d}$, in [Zh, ZhPas1], the homogenization problems in a bounded domain $\mathcal{O} \subset \mathbb{R}^{d}$ with the Dirichlet or Neumann boundary condition were studied. Further results of V. V. Zhikov and S. E. Pastukhova can be found in the survey ZhPas4.

The operator error estimates for homogenization of the Dirichlet and Neumann problems for second order elliptic equation in a bounded domain were studied by many authors, see [ZhPas1, Gr1, Gr2, KeLiS, PSu, Su4, Su5]. The detailed survey can be found in the introduction to the paper [MSu3]. In [MSu3], approximations for the resolvent of the operator (0.1) were obtained:

$$
\begin{aligned}
& \left\|\left(B_{D, \varepsilon}-\zeta I\right)^{-1}-\left(B_{D}^{0}-\zeta I\right)^{-1}\right\|_{L_{2}(\mathcal{O}) \rightarrow L_{2}(\mathcal{O})} \leqslant C(\phi) \varepsilon|\zeta|^{-1 / 2}, \\
& \left\|\left(B_{D, \varepsilon}-\zeta I\right)^{-1}-\left(B_{D}^{0}-\zeta I\right)^{-1}-\varepsilon K_{D}(\varepsilon ; \zeta)\right\|_{L_{2}(\mathcal{O}) \rightarrow H^{1}(\mathcal{O})} \leqslant C(\phi)\left(\varepsilon^{1 / 2}|\zeta|^{-1 / 4}+\varepsilon\right),
\end{aligned}
$$

$\zeta \in \mathbb{C} \backslash \mathbb{R}_{+},|\zeta| \geqslant 1$. The values $C(\phi)$ are controlled explicitly in terms of the problem data and the angle $\phi=\arg \zeta$. (For $\zeta$ fixed, close results were obtained by $\mathrm{Q}$. Xu $[\mathrm{Xu}]$.)

To parabolic problems in the whole space $\mathbb{R}^{d}$, spectral method was applied by T. A. Suslina [Su1, Su2]. It was obtained that

$$
\begin{aligned}
& \left\|e^{-t A_{\varepsilon}}-e^{-t A^{0}}\right\|_{L_{2}\left(\mathbb{R}^{d}\right) \rightarrow L_{2}\left(\mathbb{R}^{d}\right)} \leqslant C \varepsilon\left(t+\varepsilon^{2}\right)^{-1 / 2}, \quad t \geqslant 0, \\
& \left\|e^{-t A_{\varepsilon}}-e^{-t A^{0}}-\varepsilon \mathfrak{K}(\varepsilon ; t)\right\|_{L_{2}\left(\mathbb{R}^{d}\right) \rightarrow H^{1}\left(\mathbb{R}^{d}\right)} \leqslant C \varepsilon\left(t^{-1 / 2}+t^{-1}\right), \quad t \geqslant \varepsilon^{2} .
\end{aligned}
$$

By the shift method, these estimates were proven by V. V. Zhikov and S. E. Pastukhova [ZhPas2]. Later, results (0.11) and (0.12) were transferred to the operator $B_{\varepsilon}$ by the author [M1].

Homogenization of the first initial-boundary value problem for a parabolic equation involving the operator $b(\mathbf{D})^{*} g^{\varepsilon}(\mathbf{x}) b(\mathbf{D})$ or the operator (0.1) was studied by Yu. M. Meshkova and T. A. Suslina in [MSu2] and [MSu5], respectively. The method was based on the identity

$$
e^{-t B_{D, \varepsilon}}=-\frac{1}{2 \pi i} \int_{\gamma} e^{-\zeta t}\left(B_{D, \varepsilon}-\zeta I\right)^{-1} d \zeta
$$

and estimates (0.9) and (0.10). Here $\gamma \subset \mathbb{C}$ is a contour enclosing the spectrum of the operator $B_{D, \varepsilon}$ in the positive direction. Recall that, according to the classical Trotter-Kato theorem (see, e. g., [Sa, Chapter X, Theorem 1.1]), the strong convergence of semigroups follows from the strong convergence of the corresponding resolvents, while in [MSu5] approximations in the uniform operator topology with explicit error estimates were obtained. Let us mention the recent work [ChEl], where the Trotter-Kato theorem was transferred to weak and uniform operator topologies and the results were applied to homogenization of the parabolic equations (without operator error estimates).

In [BSu4, M2, DSu], the spectral approach was applied to the hyperbolic systems. In [BSu4], for $t \in \mathbb{R}$ it was obtained that

$$
\begin{aligned}
& \left\|\cos \left(t A_{\varepsilon}^{1 / 2}\right)-\cos \left(t\left(A^{0}\right)^{1 / 2}\right)\right\|_{H^{2}\left(\mathbb{R}^{d}\right) \rightarrow L_{2}\left(\mathbb{R}^{d}\right)} \leqslant C \varepsilon(1+|t|), \\
& \left\|A_{\varepsilon}^{-1 / 2} \sin \left(t A_{\varepsilon}^{1 / 2}\right)-\left(A^{0}\right)^{-1 / 2} \sin \left(t\left(A^{0}\right)^{1 / 2}\right)\right\|_{H^{2}\left(\mathbb{R}^{d}\right) \rightarrow L_{2}\left(\mathbb{R}^{d}\right)} \leqslant C \varepsilon(1+|t|)^{2} .
\end{aligned}
$$

In [M2], estimate (0.14) was refined with respect to the type of the operator norm:

$$
\left\|A_{\varepsilon}^{-1 / 2} \sin \left(t A_{\varepsilon}^{1 / 2}\right)-\left(A^{0}\right)^{-1 / 2} \sin \left(t\left(A^{0}\right)^{1 / 2}\right)\right\|_{H^{1}\left(\mathbb{R}^{d}\right) \rightarrow L_{2}\left(\mathbb{R}^{d}\right)} \leqslant C \varepsilon(1+|t|),
$$


$t \in \mathbb{R}$, and approximation for the operator $A_{\varepsilon}^{-1 / 2} \sin \left(t A_{\varepsilon}^{1 / 2}\right)$ in the $\left(H^{2} \rightarrow H^{1}\right)$-operator norm was obtained

$$
\begin{aligned}
& \left\|A_{\varepsilon}^{-1 / 2} \sin \left(t A_{\varepsilon}^{1 / 2}\right)-\left(A^{0}\right)^{-1 / 2} \sin \left(t\left(A^{0}\right)^{1 / 2}\right)-\varepsilon \mathrm{K}(\varepsilon ; t)\right\|_{H^{2}\left(\mathbb{R}^{d}\right) \rightarrow H^{1}\left(\mathbb{R}^{d}\right)} \\
& \quad \leqslant C \varepsilon(1+|t|), \quad t \in \mathbb{R} .
\end{aligned}
$$

Here $\mathrm{K}(\varepsilon ; t)$ is the corrector. In [DSu], the sharpness of estimates (0.13) and (0.15) with respect to the type of the norm was proven in the general case.

The correctors in estimates (0.8), (0.12), and (0.16) have a similar structure. So, it seems natural to expect that the cosine operator also can be approximated in the energy norm with a similar corrector. However, in BrOtFMu it was observed that even the fact of the norm convergence is true only for the very special choice of the initial data. The argument used in [BrOtFMu $]$ is the following: the convergence of the energy does not occur in the general situation. But the solution can be splitted into two parts: the first one is designed so that the corresponding energy converges to the energy for the effective equation and the second part tends to zero *-weakly in $L_{\infty}\left((0, T) ; H_{0}^{1}(\mathcal{O})\right) \cap W^{1, \infty}\left((0, T) ; L_{2}(\mathcal{O})\right)$. In our considerations, we deal only with the first part. This case corresponds to estimate (0.6). In the general case, some approximations with the corrector were obtained in [BraLe, CaDiCoCalMaMarG]. Their results can not be written in the uniform operator topology. The corresponding corrector is non-local because of the dispersion of waves in the inhomogeneous media. Dispersion effects for homogenization of the wave equation were discussed in [ABriV, ConOrV, ConSaMaBalV], but the operator error estimates were not studied.

Let us also mention a recent preprint [CooSav, where (independently of the present work) the homogenization of the attractors of the quasi-linear damped wave equation was derived from the estimate of the form (0.9) for the operator $B_{D, \varepsilon}=-\operatorname{div} g^{\varepsilon}(\mathbf{x}) \nabla$ (and $\zeta=0$ ). But the results of [CooSav can not be written in the uniform operator topology. Thus, operator error estimates for homogenization of hyperbolic systems in a bounded domain were not previously known.

0.4. Method. The present work develops the method of [MSu2, MSu5]. We deduce operator error estimates for non-stationary problem from the elliptic results via the inverse Laplace transform. (Surely, the Laplace transform had been applied for homogenization of hyperbolic problems previously, see [BeLPap, Chapter 2, Subsec. 3.9], [Sa, Chapter V, Sec. 6], and [Pas, ZhPas3]. We also note that the non-stationary Maxwell system was studied by using the Laplace transform in [ZhKO, Chapter IV]. But the operator error estimates were not discussed in the books and papers listed above.)

The method is based on the identity

$$
\cos \left(t B_{D, \varepsilon}^{1 / 2}\right) B_{D, \varepsilon}^{-2}=-\frac{t^{2}}{2} B_{D, \varepsilon}^{-1}+B_{D, \varepsilon}^{-2}+\frac{1}{2 \pi i} \int_{\operatorname{Re} \lambda=\sqrt{c}} \lambda^{-3}\left(B_{D, \varepsilon}+\lambda^{2}\right)^{-1} e^{\lambda t} d \lambda,
$$

$c>0$, and on using the approximations of the operator $\left(B_{D, \varepsilon}-\zeta I\right)^{-1}, \zeta \in \mathbb{C} \backslash \mathbb{R}_{+}$, with the error estimates that are two-parametric with respect to $\varepsilon$ and $\zeta$. The required approximations were obtained in $\mathrm{MSu} 3$. Combining (0.17), the similar identity for the effective operator, and estimate (0.9), we obtain inequality (0.3). To derive estimate (0.4) from (0.3), we use the representations

$$
\begin{aligned}
& B_{D, \varepsilon}^{-1 / 2} \sin \left(t B_{D, \varepsilon}^{1 / 2}\right)=\int_{0}^{t} \cos \left(\tau B_{D, \varepsilon}^{1 / 2}\right) d \tau \\
& \left(B_{D}^{0}\right)^{-1 / 2} \sin \left(t\left(B_{D}^{0}\right)^{1 / 2}\right)=\int_{0}^{t} \cos \left(\tau\left(B_{D}^{0}\right)^{1 / 2}\right) d \tau .
\end{aligned}
$$


The approximation with the corrector for the operator $B_{D, \varepsilon}^{-1} \cos \left(t B_{D, \varepsilon}^{1 / 2}\right)$ follows from (0.17), the similar identity for the effective operator, and estimate (0.10). Using this approximation, identities (0.18), (0.19), and homogenization results for the resolvent, we obtain inequality (0.5).

The presence of the operator $\left(B_{D}^{0}\right)^{-2}$ in estimates $(\underline{0.3)}-(0.5)$ is caused by the method of investigation. Because of this factor, the initial data and the right-hand side in (0.2) are subject to the following restrictions:

$$
\boldsymbol{\varphi}, \boldsymbol{\psi} \in \operatorname{Dom}\left(B_{D}^{0}\right)^{2}, \quad \mathbf{F} \in L_{1, \operatorname{loc}}\left(\mathbb{R} ; \operatorname{Dom}\left(B_{D}^{0}\right)^{2}\right) .
$$

If $\partial \mathcal{O} \in C^{3,1}$, then Dom $\left(B_{D}^{0}\right)^{2}$ can be considered as the subspace of $H^{4}\left(\mathcal{O} ; \mathbb{C}^{n}\right)$. Thus, the initial data and the right-hand side of the equation (0.2) are required to be more smooth compared with the data for the problems in the whole space. Apparently, the results of the present paper are not sharp with respect to the classes of smoothness for the initial data and the right-hand side of the equation. However, it seems that the applied technique does not allow to improve the results.

0.5. Plan of the paper. The paper consists of three sections and introduction. In Section 1 , the class of the operators $B_{D, \varepsilon}$ is described, the effective operator $B_{D}^{0}$ is defined and the approximations for the resolvent $\left(B_{D, \varepsilon}-\zeta I\right)^{-1}$ are formulated. Section 2 contains the main results of the paper. Their proofs can be found in Section 3 ,

0.6. Notation. Let $\mathfrak{H}$ and $\mathfrak{H}_{*}$ be complex separable Hilbert spaces. The symbols $(\cdot, \cdot)_{\mathfrak{H}}$ and $\|\cdot\|_{\mathfrak{H}}$ denote the inner product and the norm in $\mathfrak{H}$, respectively; the symbol $\|\cdot\|_{\mathfrak{H} \rightarrow \mathfrak{H}_{*}}$ means the norm of the linear continuous operator from $\mathfrak{H}$ to $\mathfrak{H}_{*}$.

We use the notation $\mathbb{Z}_{+}$for the set of non-negative integers and $\mathbb{R}_{+}$for the positive half-line $[0, \infty)$.

The symbols $\langle\cdot, \cdot\rangle$ and $|\cdot|$ stand for the inner product and the norm in $\mathbb{C}^{n}$, respectively; $\mathbf{1}_{n}$ is the identity $(n \times n)$-matrix. If $a$ is $(m \times n)$-matrix, then the symbol $|a|$ denotes the norm of the matrix $a$ viewed as the operator from $\mathbb{C}^{n}$ to $\mathbb{C}^{m}$. For $z \in \mathbb{C}$, by $z^{*}$ we denote the complex conjugate number. (We use such non-standard notation, because the upper line denotes the mean value of a periodic function over the cell of periodicity.) We use the notation $\mathbf{x}=\left(x_{1}, \ldots, x_{d}\right) \in \mathbb{R}^{d}, i D_{j}=\partial_{j}=\partial / \partial x_{j}, j=1, \ldots, d, \mathbf{D}=-i \nabla=\left(D_{1}, \ldots, D_{d}\right)$. The classes $L_{p}$ of $\mathbb{C}^{n}$-valued functions in a domain $\mathcal{O} \subset \mathbb{R}^{d}$ are denoted by $L_{p}\left(\mathcal{O} ; \mathbb{C}^{n}\right), 1 \leqslant p \leqslant \infty$. The Sobolev spaces of $\mathbb{C}^{n}$-valued functions in a domain $\mathcal{O} \subset \mathbb{R}^{d}$ are denoted by $H^{s}\left(\mathcal{O} ; \mathbb{C}^{n}\right)$. By $H_{0}^{1}\left(\mathcal{O} ; \mathbb{C}^{n}\right)$ we denote the closure of the class $C_{0}^{\infty}\left(\mathcal{O} ; \mathbb{C}^{n}\right)$ in the space $H^{1}\left(\mathcal{O} ; \mathbb{C}^{n}\right)$. For $n=1$, we simply write $L_{p}(\mathcal{O}), H^{s}(\mathcal{O})$ and so on, but, sometimes, if this does not lead to confusion, we use such simple notation for the spaces of vector-valued or matrix-valued functions. The symbol $L_{p}((0, T) ; \mathfrak{H}), 1 \leqslant p \leqslant \infty$, means the $L_{p}$-space of $\mathfrak{H}$-valued functions on the interval $(0, T)$.

Various constants in estimates are denoted by $c, \mathfrak{c}, C, \mathcal{C}, \mathscr{C}, \mathfrak{C}$ (possibly, with indices and marks).

Acknowledgement. The author is deeply grateful to T. A. Suslina for her attention to this work.

\section{Homogenization Results for the Elliptic Dirichlet PRoBlem}

1.1. Lattices in $\mathbb{R}^{d}$. Let $\Gamma \subset \mathbb{R}^{d}$ be the lattice generated by the basis $\mathbf{a}_{1}, \ldots, \mathbf{a}_{d} \in \mathbb{R}^{d}$ :

$$
\Gamma=\left\{\mathbf{a} \in \mathbb{R}^{d}: \mathbf{a}=\sum_{j=1}^{d} \nu_{j} \mathbf{a}_{j}, \nu_{j} \in \mathbb{Z}\right\},
$$


and let $\Omega$ be the elementary cell of the lattice $\Gamma$ :

$$
\Omega=\left\{\mathbf{x} \in \mathbb{R}^{d}: \mathbf{x}=\sum_{j=1}^{d} \tau_{j} \mathbf{a}_{j},-\frac{1}{2}<\tau_{j}<\frac{1}{2}\right\} .
$$

By $|\Omega|$ we denote the Lebesgue measure of the cell $\Omega$ : $|\Omega|=$ meas $\Omega$. Set $2 r_{1}:=\operatorname{diam} \Omega$.

The basis $\mathbf{b}_{1}, \ldots, \mathbf{b}_{d} \in \mathbb{R}^{d}$, dual to $\mathbf{a}_{1}, \ldots, \mathbf{a}_{d}$, is defined by the relations $\left\langle\mathbf{b}_{j}, \mathbf{a}_{i}\right\rangle=2 \pi \delta_{j i}$. This basis generates the lattice $\widetilde{\Gamma}$ dual to $\Gamma$. Denote $2 r_{0}:=\min _{0 \neq \mathbf{b} \in \widetilde{\Gamma}}|\mathbf{b}|$.

Let $\widetilde{H}^{1}(\Omega)$ be the subspace of functions from $H^{1}(\Omega)$ whose $\Gamma$-periodic extension to $\mathbb{R}^{d}$ belongs to $H_{\text {loc }}^{1}\left(\mathbb{R}^{d}\right)$. If $\Phi(\mathbf{x})$ is a $\Gamma$-periodic matrix-valued function in $\mathbb{R}^{d}$, we put $\Phi^{\varepsilon}(\mathbf{x}):=$ $\Phi(\mathbf{x} / \varepsilon), \varepsilon>0 ; \bar{\Phi}:=|\Omega|^{-1} \int_{\Omega} \Phi(\mathbf{x}) d \mathbf{x}, \underline{\Phi}:=\left(|\Omega|^{-1} \int_{\Omega} \Phi(\mathbf{x})^{-1} d \mathbf{x}\right)^{-1}$. Here in the definition of $\bar{\Phi}$ it is assumed that $\Phi \in L_{1, \text { loc }}\left(\mathbb{R}^{d}\right)$; in the definition of $\underline{\Phi}$ it is assumed that the matrixvalued function $\Phi$ is square and non-degenerate, and $\Phi^{-1} \in L_{1, \text { loc }}\left(\mathbb{R}^{d}\right)$. By $\left[\Phi^{\varepsilon}\right]$ we denote the operator of multiplication by the matrix-valued function $\Phi^{\varepsilon}(\mathbf{x})$.

1.2. The Steklov smoothing. The Steklov smoothing operator $S_{\varepsilon}^{(k)}$ acts in the space $L_{2}\left(\mathbb{R}^{d} ; \mathbb{C}^{k}\right)$ (where $k \in \mathbb{N}$ ) and is defined by

$$
\left(S_{\varepsilon}^{(k)} \mathbf{u}\right)(\mathbf{x})=|\Omega|^{-1} \int_{\Omega} \mathbf{u}(\mathbf{x}-\varepsilon \mathbf{z}) d \mathbf{z}, \quad \mathbf{u} \in L_{2}\left(\mathbb{R}^{d} ; \mathbb{C}^{k}\right)
$$

We will omit the index $k$ in the notation and write simply $S_{\varepsilon}$. Obviously, $S_{\varepsilon} \mathbf{D}^{\alpha} \mathbf{u}=\mathbf{D}^{\alpha} S_{\varepsilon} \mathbf{u}$ for $\mathbf{u} \in H^{\sigma}\left(\mathbb{R}^{d} ; \mathbb{C}^{k}\right)$ and any multiindex $\alpha$ such that $|\alpha| \leqslant \sigma$. We need the following properties of the operator $S_{\varepsilon}$ (see [ZhPas1, Lemmas 1.1 and 1.2] or [PSu, Propositions 3.1 and 3.2]).

Proposition 1.1. For any function $\mathbf{u} \in H^{1}\left(\mathbb{R}^{d} ; \mathbb{C}^{k}\right)$ we have

$$
\left\|S_{\varepsilon} \mathbf{u}-\mathbf{u}\right\|_{L_{2}\left(\mathbb{R}^{d}\right)} \leqslant \varepsilon r_{1}\|\mathbf{D u}\|_{L_{2}\left(\mathbb{R}^{d}\right)},
$$

where $2 r_{1}=\operatorname{diam} \Omega$.

Proposition 1.2. Let $\Phi$ be a $\Gamma$-periodic function in $\mathbb{R}^{d}$ such that $\Phi \in L_{2}(\Omega)$. Then the operator $\left[\Phi^{\varepsilon}\right] S_{\varepsilon}$ is continuous in $L_{2}\left(\mathbb{R}^{d}\right)$ and

$$
\left\|\left[\Phi^{\varepsilon}\right] S_{\varepsilon}\right\|_{L_{2}\left(\mathbb{R}^{d}\right) \rightarrow L_{2}\left(\mathbb{R}^{d}\right)} \leqslant|\Omega|^{-1 / 2}\|\Phi\|_{L_{2}(\Omega)} .
$$

1.3. The operator $A_{D, \varepsilon}$. Let $\mathcal{O} \subset \mathbb{R}^{d}$ be a bounded domain of class $C^{1,1}$. In $L_{2}\left(\mathcal{O} ; \mathbb{C}^{n}\right)$, we consider the operator $A_{D, \varepsilon}$ formally given by the differential expression $A_{\varepsilon}=b(\mathbf{D})^{*} g^{\varepsilon}(\mathbf{x}) b(\mathbf{D})$ with the Dirichlet condition on $\partial \mathcal{O}$. (We agree to mark a differential operator with the Dirichlet condition and its quadratic form (but not a formal differential expression corresponding to the operator) by the lower index „ $\left.D^{“}.\right)$ Here $g(\mathbf{x})$ is a $\Gamma$-periodic Hermitian $(m \times m)$-matrix-valued function (in general, with complex entries). Assume that $g(\mathbf{x})>0$ and $g, g^{-1} \in L_{\infty}\left(\mathbb{R}^{d}\right)$. The differential operator $b(\mathbf{D})$ has the form $b(\mathbf{D})=\sum_{j=1}^{d} b_{j} D_{j}$, where $b_{j}, j=1, \ldots, d$, are constant matrices of the size $m \times n$ (in general, with complex entries). Assume that $m \geqslant n$ and that the symbol $b(\boldsymbol{\xi})=\sum_{j=1}^{d} b_{j} \xi_{j}$ of the operator $b(\mathbf{D})$ has maximal rank: $\operatorname{rank} b(\boldsymbol{\xi})=n$ for $0 \neq \boldsymbol{\xi} \in \mathbb{R}^{d}$. This is equivalent to the existence of constants $\alpha_{0}$ and $\alpha_{1}$ such that

$$
\alpha_{0} \mathbf{1}_{n} \leqslant b(\boldsymbol{\theta})^{*} b(\boldsymbol{\theta}) \leqslant \alpha_{1} \mathbf{1}_{n}, \quad \boldsymbol{\theta} \in \mathbb{S}^{d-1} ; \quad 0<\alpha_{0} \leqslant \alpha_{1}<\infty
$$

By (1.2),

$$
\left|b_{j}\right| \leqslant \alpha_{1}^{1 / 2}, \quad j=1, \ldots, d .
$$

The precise definition of the operator $A_{D, \varepsilon}$ is given via the quadratic form

$$
\mathfrak{a}_{D, \varepsilon}[\mathbf{u}, \mathbf{u}]=\int_{\mathcal{O}}\left\langle g^{\varepsilon}(\mathbf{x}) b(\mathbf{D}) \mathbf{u}, b(\mathbf{D}) \mathbf{u}\right\rangle d \mathbf{x}, \quad \mathbf{u} \in H_{0}^{1}\left(\mathcal{O} ; \mathbb{C}^{n}\right) .
$$


Extending the function $\mathbf{u} \in H_{0}^{1}\left(\mathcal{O} ; \mathbb{C}^{n}\right)$ by zero onto $\mathbb{R}^{d} \backslash \mathcal{O}$ and taking (1.2) into account, we obtain

$$
\alpha_{0}\left\|g^{-1}\right\|_{L_{\infty}}^{-1}\|\mathbf{D u}\|_{L_{2}(\mathcal{O})}^{2} \leqslant \mathfrak{a}_{D, \varepsilon}[\mathbf{u}, \mathbf{u}] \leqslant \alpha_{1}\|g\|_{L_{\infty}}\|\mathbf{D u}\|_{L_{2}(\mathcal{O})}^{2}, \quad \mathbf{u} \in H_{0}^{1}\left(\mathcal{O} ; \mathbb{C}^{n}\right) .
$$

1.4. Lower order terms. The operator $B_{D, \varepsilon}$. We study the self-adjoint operator $B_{D, \varepsilon}$ with the principal part $A_{\varepsilon}$. To define the lower order terms, let us introduce $\Gamma$-periodic $(n \times n)$-matrix-valued functions (in general, with complex entries) $a_{j}, j=1, \ldots, d$, such that

$$
a_{j} \in L_{\rho}(\Omega), \quad \rho=2 \text { for } d=1, \quad \rho>d \text { for } d \geqslant 2, \quad j=1, \ldots, d .
$$

Next, let $Q$ be the $\Gamma$-periodic Hermitian $(n \times n)$-matrix-valued function (with complex entries) such that

$$
Q \in L_{s}(\Omega), \quad s=1 \text { for } d=1, \quad s>d / 2 \text { for } d \geqslant 2 .
$$

By the Sobolev embedding theorem, conditions imposed on $\rho$ and $s$ guarantee that the lower terms of the operator $B_{D, \varepsilon}$ are strongly subordinate to its principal part $A_{\varepsilon}$.

For convenience of further references, the following set of variables is called the ,,problem data":

$$
d, m, n, \rho, s ; \alpha_{0}, \alpha_{1},\|g\|_{L_{\infty}},\left\|g^{-1}\right\|_{L_{\infty}},\left\|a_{j}\right\|_{L_{\rho}(\Omega)}, j=1, \ldots, d ;\|Q\|_{L_{s}(\Omega)}
$$

the parameters of the lattice $\Gamma$; the domain $\mathcal{O}$.

In $L_{2}\left(\mathcal{O} ; \mathbb{C}^{n}\right)$, we consider the operator $B_{D, \varepsilon}, 0<\varepsilon \leqslant 1$, formally given by the differential expression

$$
B_{\varepsilon}=b(\mathbf{D})^{*} g^{\varepsilon}(\mathbf{x}) b(\mathbf{D})+\sum_{j=1}^{d}\left(a_{j}^{\varepsilon}(\mathbf{x}) D_{j}+D_{j} a_{j}^{\varepsilon}(\mathbf{x})^{*}\right)+Q^{\varepsilon}(\mathbf{x})+\lambda I
$$

with the Dirichet boundary condition. Here the constant $\lambda$ is chosen so that (see (1.14) below) the operator $B_{D, \varepsilon}$ is positive definite. The precise definition of the operator $B_{D, \varepsilon}$ is given via the quadratic form

$$
\begin{aligned}
\mathfrak{b}_{D, \varepsilon}[\mathbf{u}, \mathbf{u}] & =\left(g^{\varepsilon} b(\mathbf{D}) \mathbf{u}, b(\mathbf{D}) \mathbf{u}\right)_{L_{2}(\mathcal{O})}+2 \operatorname{Re} \sum_{j=1}^{d}\left(a_{j}^{\varepsilon} D_{j} \mathbf{u}, \mathbf{u}\right)_{L_{2}(\mathcal{O})} \\
& +\left(Q^{\varepsilon} \mathbf{u}, \mathbf{u}\right)_{L_{2}(\mathcal{O})}+\lambda(\mathbf{u}, \mathbf{u})_{L_{2}(\mathcal{O})}, \quad \mathbf{u} \in H_{0}^{1}\left(\mathcal{O} ; \mathbb{C}^{n}\right) .
\end{aligned}
$$

Let us check that the form $\mathfrak{b}_{D, \varepsilon}$ is closed. By the Hölder inequality and the Sobolev embedding theorem, it can be shown (see [Su3, (5.11)-(5.14)]) that for any $\nu>0$ there exist constants $C_{j}(\nu)>0$ such that

$$
\left\|a_{j}^{*} \mathbf{u}\right\|_{L_{2}\left(\mathbb{R}^{d}\right)}^{2} \leqslant \nu\|\mathbf{D u}\|_{L_{2}\left(\mathbb{R}^{d}\right)}^{2}+C_{j}(\nu)\|\mathbf{u}\|_{L_{2}\left(\mathbb{R}^{d}\right)}^{2}, \quad \mathbf{u} \in H^{1}\left(\mathbb{R}^{d} ; \mathbb{C}^{n}\right), \quad j=1, \ldots, d .
$$

By the change of variables $\mathbf{y}:=\varepsilon^{-1} \mathbf{x}$ and $\mathbf{u}(\mathbf{x})=: \mathbf{v}(\mathbf{y})$, we deduce

$$
\begin{aligned}
\left\|\left(a_{j}^{\varepsilon}\right)^{*} \mathbf{u}\right\|_{L_{2}\left(\mathbb{R}^{d}\right)}^{2} & =\int_{\mathbb{R}^{d}}\left|a_{j}\left(\varepsilon^{-1} \mathbf{x}\right)^{*} \mathbf{u}(\mathbf{x})\right|^{2} d \mathbf{x}=\varepsilon^{d} \int_{\mathbb{R}^{d}}\left|a_{j}(\mathbf{y})^{*} \mathbf{v}(\mathbf{y})\right|^{2} d \mathbf{y} \\
& \leqslant \varepsilon^{d} \nu \int_{\mathbb{R}^{d}}\left|\mathbf{D}_{\mathbf{y}} \mathbf{v}(\mathbf{y})\right|^{2} d \mathbf{y}+\varepsilon^{d} C_{j}(\nu) \int_{\mathbb{R}^{d}}|\mathbf{v}(\mathbf{y})|^{2} d \mathbf{y} \\
& \leqslant \nu\|\mathbf{D u}\|_{L_{2}\left(\mathbb{R}^{d}\right)}^{2}+C_{j}(\nu)\|\mathbf{u}\|_{L_{2}\left(\mathbb{R}^{d}\right)}^{2}, \quad \mathbf{u} \in H^{1}\left(\mathbb{R}^{d} ; \mathbb{C}^{n}\right), \quad 0<\varepsilon \leqslant 1 .
\end{aligned}
$$

Then, by (1.2), for any $\nu>0$ there exists a constant $C(\nu)>0$ such that

$$
\begin{array}{r}
\sum_{j=1}^{d}\left\|\left(a_{j}^{\varepsilon}\right)^{*} \mathbf{u}\right\|_{L_{2}\left(\mathbb{R}^{d}\right)}^{2} \leqslant \nu\left\|\left(g^{\varepsilon}\right)^{1 / 2} b(\mathbf{D}) \mathbf{u}\right\|_{L_{2}\left(\mathbb{R}^{d}\right)}^{2}+C(\nu)\|\mathbf{u}\|_{L_{2}\left(\mathbb{R}^{d}\right)}^{2}, \\
\mathbf{u} \in H^{1}\left(\mathbb{R}^{d} ; \mathbb{C}^{n}\right), \quad 0<\varepsilon \leqslant 1 .
\end{array}
$$


For $\nu$ fixed, $C(\nu)$ depends only on $d, \rho, \alpha_{0}$, on the norms $\left\|g^{-1}\right\|_{L_{\infty}},\left\|a_{j}\right\|_{L_{\rho}(\Omega)}, j=1, \ldots, d$, and on the parameters of the lattice $\Gamma$.

By $(\underline{1.2})$, for $\mathbf{u} \in H^{1}\left(\mathbb{R}^{d} ; \mathbb{C}^{n}\right)$ we have

$$
\|\mathbf{D u}\|_{L_{2}\left(\mathbb{R}^{d}\right)}^{2} \leqslant c_{1}^{2}\left\|\left(g^{\varepsilon}\right)^{1 / 2} b(\mathbf{D}) \mathbf{u}\right\|_{L_{2}\left(\mathbb{R}^{d}\right)}^{2},
$$

where $c_{1}:=\alpha_{0}^{-1 / 2}\left\|g^{-1}\right\|_{L_{\infty}}^{1 / 2}$. Combining this with (1.10), we obtain

$$
\begin{array}{r}
2\left|\operatorname{Re} \sum_{j=1}^{d}\left(D_{j} \mathbf{u},\left(a_{j}^{\varepsilon}\right)^{*} \mathbf{u}\right)_{L_{2}\left(\mathbb{R}^{d}\right)}\right| \leqslant \frac{1}{4}\left\|\left(g^{\varepsilon}\right)^{1 / 2} b(\mathbf{D}) \mathbf{u}\right\|_{L_{2}\left(\mathbb{R}^{d}\right)}^{2}+c_{2}\|\mathbf{u}\|_{L_{2}\left(\mathbb{R}^{d}\right)}^{2}, \\
\mathbf{u} \in H^{1}\left(\mathbb{R}^{d} ; \mathbb{C}^{n}\right), \quad 0<\varepsilon \leqslant 1,
\end{array}
$$

where $c_{2}:=8 c_{1}^{2} C\left(\nu_{0}\right)$ for $\nu_{0}:=2^{-6} \alpha_{0}\left\|g^{-1}\right\|_{L_{\infty}}^{-1}$.

Next, by condition (1.6) for $Q$, for any $\nu>0$ there exists a constant $C_{Q}(\nu)>0$ such that

$$
\begin{array}{r}
\left|\left(Q^{\varepsilon} \mathbf{u}, \mathbf{u}\right)_{L_{2}\left(\mathbb{R}^{d}\right)}\right| \leqslant \nu\|\mathbf{D u}\|_{L_{2}\left(\mathbb{R}^{d}\right)}^{2}+C_{Q}(\nu)\|\mathbf{u}\|_{L_{2}\left(\mathbb{R}^{d}\right)}^{2}, \\
\mathbf{u} \in H^{1}\left(\mathbb{R}^{d} ; \mathbb{C}^{n}\right), \quad 0<\varepsilon \leqslant 1 .
\end{array}
$$

For $\nu$ fixed, the constant $C_{Q}(\nu)$ is controlled in terms of $d, s,\|Q\|_{L_{s}(\Omega)}$, and the parameters of the lattice $\Gamma$.

We fix a constant $\lambda$ in (1.8) as follows:

$$
\lambda:=C_{Q}\left(\nu_{*}\right)+c_{2} \quad \text { for } \nu_{*}:=2^{-1} \alpha_{0}\left\|g^{-1}\right\|_{L_{\infty}}^{-1} .
$$

Now, we return to the form (1.9). The function $\mathbf{u} \in H_{0}^{1}\left(\mathcal{O} ; \mathbb{C}^{n}\right)$ is extended by zero to $\mathbb{R}^{d} \backslash \mathcal{O}$. From (1.4), (1.11), (1.12), (1.13) with $\nu=\nu_{*}$, and (1.14) we derive the lower estimate for the form (1.9):

$$
\mathfrak{b}_{D, \varepsilon}[\mathbf{u}, \mathbf{u}] \geqslant \frac{1}{4} \mathfrak{a}_{D, \varepsilon}[\mathbf{u}, \mathbf{u}] \geqslant c_{*}\|\mathbf{D u}\|_{L_{2}(\mathcal{O})}^{2}, \quad \mathbf{u} \in H_{0}^{1}\left(\mathcal{O} ; \mathbb{C}^{n}\right) ; \quad c_{*}:=\frac{1}{4} \alpha_{0}\left\|g^{-1}\right\|_{L_{\infty}}^{-1}
$$

Next, by (1.5), (1.12), and (1.13) with $\nu=1$,

$$
\mathfrak{b}_{D, \varepsilon}[\mathbf{u}, \mathbf{u}] \leqslant C_{*}\|\mathbf{u}\|_{H^{1}(\mathcal{O})}^{2}, \quad \mathbf{u} \in H_{0}^{1}\left(\mathcal{O} ; \mathbb{C}^{n}\right),
$$

where $C_{*}:=\max \left\{\frac{5}{4} \alpha_{1}\|g\|_{L_{\infty}}+1 ; C_{Q}(1)+\lambda+c_{2}\right\}$. Thus, the form $\mathfrak{b}_{D, \varepsilon}$ is closed. The corresponding self-adjoint operator in $L_{2}\left(\mathcal{O} ; \mathbb{C}^{n}\right)$ is denoted by $B_{D, \varepsilon}$.

By the Friedrichs inequality, from (1.15) we deduce that

$$
\mathfrak{b}_{D, \varepsilon}[\mathbf{u}, \mathbf{u}] \geqslant c_{*}(\operatorname{diam} \mathcal{O})^{-2}\|\mathbf{u}\|_{L_{2}(\mathcal{O})}^{2}, \quad \mathbf{u} \in H_{0}^{1}\left(\mathcal{O} ; \mathbb{C}^{n}\right) .
$$

So, the operator $B_{D, \varepsilon}$ is positive definite. By (1.15) and (1.17),

$$
\|\mathbf{u}\|_{H^{1}(\mathcal{O})} \leqslant c_{3}\left\|B_{D, \varepsilon}^{1 / 2} \mathbf{u}\right\|_{L_{2}(\mathcal{O})}, \quad \mathbf{u} \in H_{0}^{1}\left(\mathcal{O} ; \mathbb{C}^{n}\right) ; \quad c_{3}:=c_{*}^{-1 / 2}\left(1+(\operatorname{diam} \mathcal{O})^{2}\right)^{1 / 2}
$$

We will need the following inequalities deduced from (1.17) and (1.18):

$$
\begin{aligned}
& \left\|B_{D, \varepsilon}^{-1}\right\|_{L_{2}(\mathcal{O}) \rightarrow L_{2}(\mathcal{O})} \leqslant c_{*}^{-1}(\operatorname{diam} \mathcal{O})^{2}=: \mathcal{C}_{1}, \\
& \left\|B_{D, \varepsilon}^{-1}\right\|_{L_{2}(\mathcal{O}) \rightarrow H^{1}(\mathcal{O})} \leqslant c_{3}\left\|B_{D, \varepsilon}^{-1 / 2}\right\|_{L_{2}(\mathcal{O}) \rightarrow L_{2}(\mathcal{O})} \leqslant c_{3} c_{*}^{-1 / 2} \operatorname{diam} \mathcal{O}=: \mathcal{C}_{2} .
\end{aligned}
$$

1.5. The effective matrix and its properties. The effective operator for $A_{D, \varepsilon}$ is given by the differential expression $A^{0}=b(\mathbf{D})^{*} g^{0} b(\mathbf{D})$ with the Dirichlet condition on $\partial \mathcal{O}$. Here $g^{0}$ is the constant effective matrix of the size $m \times m$. The matrix $g^{0}$ is defined in terms of the auxiliary problem on the cell. Let $\Gamma$-periodic $(n \times m)$-matrix-valued function $\Lambda(\mathbf{x})$ be the weak solution of the problem

$$
b(\mathbf{D})^{*} g(\mathbf{x})\left(b(\mathbf{D}) \Lambda(\mathbf{x})+\mathbf{1}_{m}\right)=0, \quad \int_{\Omega} \Lambda(\mathbf{x}) d \mathbf{x}=0 .
$$

Denote

$$
\widetilde{g}(\mathbf{x}):=g(\mathbf{x})\left(b(\mathbf{D}) \Lambda(\mathbf{x})+\mathbf{1}_{m}\right) .
$$


Then the effective matrix is given by the expression

$$
g^{0}:=|\Omega|^{-1} \int_{\Omega} \widetilde{g}(\mathbf{x}) d \mathbf{x} .
$$

It can be checked that the matrix $g^{0}$ is positive definite.

From (1.21) it follows that

$$
\|b(\mathbf{D}) \Lambda\|_{L_{2}(\Omega)} \leqslant|\Omega|^{1 / 2} m^{1 / 2}\|g\|_{L_{\infty}}^{1 / 2}\left\|g^{-1}\right\|_{L_{\infty}}^{1 / 2} .
$$

We also need the following estimates for the solution of problem (1.21) proven in [BSu2, (6.28) and Subsec. 7.3]:

$$
\begin{aligned}
& \|\Lambda\|_{L_{2}(\Omega)} \leqslant|\Omega|^{1 / 2} M_{1}, \quad M_{1}:=m^{1 / 2}\left(2 r_{0}\right)^{-1} \alpha_{0}^{-1 / 2}\|g\|_{L_{\infty}}^{1 / 2}\left\|g^{-1}\right\|_{L_{\infty}}^{1 / 2}, \\
& \|\mathbf{D} \Lambda\|_{L_{2}(\Omega)} \leqslant|\Omega|^{1 / 2} M_{2}, \quad M_{2}:=m^{1 / 2} \alpha_{0}^{-1 / 2}\|g\|_{L_{\infty}}^{1 / 2}\left\|g^{-1}\right\|_{L_{\infty}}^{1 / 2} .
\end{aligned}
$$

The effective matrix satisfies the estimates known as the Voigt-Reuss bracketing (see, e. g., [BSu1, Chapter 3, Theorem 1.5]).

Proposition 1.3. Let $g^{0}$ be the effective matrix (1.23). Then

$$
\underline{g} \leqslant g^{0} \leqslant \bar{g} \text {. }
$$

If $m=n$, then $g^{0}=\underline{g}$.

Inequalities (1.27) imply that

$$
\left|g^{0}\right| \leqslant\|g\|_{L_{\infty}}, \quad\left|\left(g^{0}\right)^{-1}\right| \leqslant\left\|g^{-1}\right\|_{L_{\infty}} .
$$

Now we distinguish the cases where one of the inequalities in (1.27) becomes an identity, see [BSu1, Chapter 3, Propositions 1.6 and 1.7].

Proposition 1.4. The identity $g^{0}=\bar{g}$ is equivalent to the relations

$$
b(\mathbf{D})^{*} \mathbf{g}_{k}(\mathbf{x})=0, \quad k=1, \ldots, m,
$$

where $\mathbf{g}_{k}(\mathbf{x}), k=1, \ldots, m$, are the columns of the matrix $g(\mathbf{x})$.

Proposition 1.5. The identity $g^{0}=\underline{g}$ is equivalent to the relations

$$
\mathbf{l}_{k}(\mathbf{x})=\mathbf{l}_{k}^{0}+b(\mathbf{D}) \mathbf{w}_{k}, \quad \mathbf{l}_{k}^{0} \in \mathbb{C}^{m}, \quad \mathbf{w}_{k} \in \widetilde{H}^{1}\left(\Omega ; \mathbb{C}^{m}\right), \quad k=1, \ldots, m,
$$

where $\mathbf{l}_{k}(\mathbf{x}), k=1, \ldots, m$, are the columns of the matrix $g(\mathbf{x})^{-1}$.

1.6. The effective operator. To describe homogenization procedure for the lower order terms of the operator $B_{D, \varepsilon}$, we need another cell problem. Let $\widetilde{\Lambda}(\mathbf{x})$ be the $\Gamma$-periodic $(n \times n)$-matrix-valued solution of the problem

$$
b(\mathbf{D})^{*} g(\mathbf{x}) b(\mathbf{D}) \widetilde{\Lambda}(\mathbf{x})+\sum_{j=1}^{d} D_{j} a_{j}(\mathbf{x})^{*}=0, \quad \int_{\Omega} \widetilde{\Lambda}(\mathbf{x}) d \mathbf{x}=0 .
$$

(The equation is understood in the weak sense.) The following estimates were proven in [Su3, (7.51), (7.52)]:

$$
\begin{aligned}
& \|b(\mathbf{D}) \widetilde{\Lambda}\|_{L_{2}(\Omega)} \leqslant C_{a} n^{1 / 2} \alpha_{0}^{-1 / 2}\left\|g^{-1}\right\|_{L_{\infty}}, \\
& \|\widetilde{\Lambda}\|_{L_{2}(\Omega)} \leqslant\left(2 r_{0}\right)^{-1} C_{a} n^{1 / 2} \alpha_{0}^{-1}\left\|g^{-1}\right\|_{L_{\infty}}, \\
& \|\mathbf{D} \widetilde{\Lambda}\|_{L_{2}(\Omega)} \leqslant C_{a} n^{1 / 2} \alpha_{0}^{-1}\left\|g^{-1}\right\|_{L_{\infty}} .
\end{aligned}
$$

Here $C_{a}^{2}=\sum_{j=1}^{d} \int_{\Omega}\left|a_{j}(\mathbf{x})\right|^{2} d \mathbf{x}$. 
Next, we define the constant matrices $V$ and $W$ as follows:

$$
\begin{aligned}
& V:=|\Omega|^{-1} \int_{\Omega}(b(\mathbf{D}) \Lambda(\mathbf{x}))^{*} g(\mathbf{x})(b(\mathbf{D}) \widetilde{\Lambda}(\mathbf{x})) d \mathbf{x}, \\
& W:=|\Omega|^{-1} \int_{\Omega}(b(\mathbf{D}) \widetilde{\Lambda}(\mathbf{x}))^{*} g(\mathbf{x})(b(\mathbf{D}) \widetilde{\Lambda}(\mathbf{x})) d \mathbf{x} .
\end{aligned}
$$

In $L_{2}\left(\mathcal{O} ; \mathbb{C}^{n}\right)$, consider the quadratic form

$$
\begin{aligned}
\mathfrak{b}_{D}^{0}[\mathbf{u}, \mathbf{u}] & =\left(g^{0} b(\mathbf{D}) \mathbf{u}, b(\mathbf{D}) \mathbf{u}\right)_{L_{2}(\mathcal{O})}+2 \operatorname{Re} \sum_{j=1}^{d}\left(\overline{a_{j}} D_{j} \mathbf{u}, \mathbf{u}\right)_{L_{2}(\mathcal{O})}-2 \operatorname{Re}(V \mathbf{u}, b(\mathbf{D}) \mathbf{u})_{L_{2}(\mathcal{O})} \\
& -(W \mathbf{u}, \mathbf{u})_{L_{2}(\mathcal{O})}+(\bar{Q} \mathbf{u}, \mathbf{u})_{L_{2}(\mathcal{O})}+\lambda(\mathbf{u}, \mathbf{u})_{L_{2}(\mathcal{O})}, \quad \mathbf{u} \in H_{0}^{1}\left(\mathcal{O} ; \mathbb{C}^{n}\right) .
\end{aligned}
$$

The following estimates were obtained in [MSu3, (2.22) and (2.23)]:

$$
\begin{aligned}
& c_{*}\|\mathbf{D u}\|_{L_{2}(\mathcal{O})}^{2} \leqslant \mathfrak{b}_{D}^{0}[\mathbf{u}, \mathbf{u}] \leqslant c_{4}\|\mathbf{u}\|_{H^{1}(\mathcal{O})}^{2}, \quad \mathbf{u} \in H_{0}^{1}\left(\mathcal{O} ; \mathbb{C}^{n}\right), \\
& \mathfrak{b}_{D}^{0}[\mathbf{u}, \mathbf{u}] \geqslant c_{*}(\operatorname{diam} \mathcal{O})^{-2}\|\mathbf{u}\|_{L_{2}(\mathcal{O})}^{2}, \quad \mathbf{u} \in H_{0}^{1}\left(\mathcal{O} ; \mathbb{C}^{n}\right)
\end{aligned}
$$

Here the constant $c_{4}$ depends only on the problem data (1.7). By $B_{D}^{0}$ we denote the selfadjoint operator in $L_{2}\left(\mathcal{O} ; \mathbb{C}^{n}\right)$ corresponding to the form $\mathfrak{b}_{D}^{0}$. Combining (1.37) and (1.38), we obtain

$$
\|\mathbf{u}\|_{H^{1}(\mathcal{O})} \leqslant c_{3}\left\|\left(B_{D}^{0}\right)^{1 / 2} \mathbf{u}\right\|_{L_{2}(\mathcal{O})}, \quad \mathbf{u} \in H_{0}^{1}\left(\mathcal{O} ; \mathbb{C}^{n}\right),
$$

where $c_{3}$ is the constant from (1.18). By (1.38) and (1.39),

$$
\begin{aligned}
& \left\|\left(B_{D}^{0}\right)^{-1}\right\|_{L_{2}(\mathcal{O}) \rightarrow L_{2}(\mathcal{O})} \leqslant \mathcal{C}_{1}, \\
& \left\|\left(B_{D}^{0}\right)^{-1}\right\|_{L_{2}(\mathcal{O}) \rightarrow H^{1}(\mathcal{O})} \leqslant \mathcal{C}_{2} .
\end{aligned}
$$

Here the constants $\mathcal{C}_{1}$ and $\mathcal{C}_{2}$ are the same as in (1.19) and (1.20).

By the condition $\partial \mathcal{O} \in C^{1,1}$, the operator $B_{D}^{0}$ is defined by the differential expression

$$
B^{0}=b(\mathbf{D})^{*} g^{0} b(\mathbf{D})-b(\mathbf{D})^{*} V-V^{*} b(\mathbf{D})+\sum_{j=1}^{d}\left(\overline{a_{j}+a_{j}^{*}}\right) D_{j}-W+\bar{Q}+\lambda I
$$

on the domain $H^{2}\left(\mathcal{O} ; \mathbb{C}^{n}\right) \cap H_{0}^{1}\left(\mathcal{O} ; \mathbb{C}^{n}\right)$, and

$$
\left\|\left(B_{D}^{0}\right)^{-1}\right\|_{L_{2}(\mathcal{O}) \rightarrow H^{2}(\mathcal{O})} \leqslant \mathcal{C}_{3} .
$$

Here the constant $\mathcal{C}_{3}$ depends only on the problem data (1.7). To justify this fact, we refer to the theorems about regularity of solutions of the strongly elliptic systems (see [McL, Chapter 4]).

Remark 1.6. Instead of the condition $\partial \mathcal{O} \in C^{1,1}$, one could impose the following implicit condition: a bounded Lipschitz domain $\mathcal{O} \subset \mathbb{R}^{d}$ is such that estimate (1.42) holds. For such domain the main results of the paper in the operator terms (see Theorems [2.1, 2.2, and 2.3) remain true. In the case of scalar elliptic operators, wide conditions on $\partial \mathcal{O}$ ensuring estimate (1.42) can be found in [KoE] and [MaSh, Chapter 7] (in particular, it suffices to assume that $\left.\partial \mathcal{O} \in C^{\alpha}, \alpha>3 / 2\right)$.

Lemma 1.7. Let $B^{0}$ be the differential expression (1.41). Then for $\boldsymbol{\Phi} \in H^{4}\left(\mathcal{O} ; \mathbb{C}^{n}\right)$ we have

$$
\left\|\left(B^{0}\right)^{2} \boldsymbol{\Phi}\right\|_{L_{2}(\mathcal{O})} \leqslant \mathfrak{C}\|\boldsymbol{\Phi}\|_{H^{4}(\mathcal{O})},
$$

where the constant $\mathfrak{C}$ depends only on the problem data (1.7). 
Proof. By (1.3), (1.28), and (1.41), for $\Psi \in H^{2}\left(\mathcal{O} ; \mathbb{C}^{n}\right)$ we have

$$
\begin{aligned}
\left\|B^{0} \boldsymbol{\Psi}\right\|_{L_{2}(\mathcal{O})} & \leqslant d \alpha_{1}\|g\|_{L_{\infty}}\left\|\mathbf{D}^{2} \boldsymbol{\Psi}\right\|_{L_{2}(\mathcal{O})}+2 \alpha_{1}^{1 / 2} d^{1 / 2}|V|\|\mathbf{D} \boldsymbol{\Psi}\|_{L_{2}(\mathcal{O})} \\
& +2\left(\sum_{j=1}^{d}\left|\overline{a_{j}}\right|^{2}\right)^{1 / 2}\|\mathbf{D} \boldsymbol{\Psi}\|_{L_{2}(\mathcal{O})}+(|W|+|\bar{Q}|+\lambda)\|\boldsymbol{\Psi}\|_{L_{2}(\mathcal{O})} .
\end{aligned}
$$

From (1.24), (1.32), and (1.35) it follows that

$$
|V| \leqslant|\Omega|^{-1}\|g\|_{L_{\infty}}\|b(\mathbf{D}) \Lambda\|_{L_{2}(\Omega)}\|b(\mathbf{D}) \widetilde{\Lambda}\|_{L_{2}(\Omega)} \leqslant C_{V},
$$

where $C_{V}:=|\Omega|^{-1 / 2} \alpha_{0}^{-1 / 2} C_{a} m^{1 / 2} n^{1 / 2}\|g\|_{L_{\infty}}^{3 / 2}\left\|g^{-1}\right\|_{L_{\infty}}^{3 / 2}$. By (1.32) and (1.36),

$$
|W| \leqslant|\Omega|^{-1}\|g\|_{L_{\infty}}\|b(\mathbf{D}) \widetilde{\Lambda}\|_{L_{2}(\Omega)}^{2} \leqslant C_{W}
$$

where $C_{W}:=|\Omega|^{-1} C_{a}^{2} n \alpha_{0}^{-1}\|g\|_{L_{\infty}}\left\|g^{-1}\right\|_{L_{\infty}}^{2}$. Obviously,

$$
\sum_{j=1}^{d}\left|\overline{a_{j}}\right|^{2} \leqslant|\Omega|^{-1} C_{a}^{2}, \quad|\bar{Q}| \leqslant|\Omega|^{-1 / s}\|Q\|_{L_{s}(\Omega)} .
$$

Bringing (1.44)-(1.47) together, we conclude

$$
\left\|B^{0} \boldsymbol{\Psi}\right\|_{L_{2}(\mathcal{O})} \leqslant C_{B}\left(\left\|\mathbf{D}^{2} \boldsymbol{\Psi}\right\|_{L_{2}(\mathcal{O})}+\|\mathbf{D} \boldsymbol{\Psi}\|_{L_{2}(\mathcal{O})}+\|\boldsymbol{\Psi}\|_{L_{2}(\mathcal{O})}\right), \quad \boldsymbol{\Psi} \in H^{2}\left(\mathcal{O} ; \mathbb{C}^{n}\right) .
$$

Here $C_{B}:=\max \left\{d \alpha_{1}\|g\|_{L_{\infty}} ; 2\left(d \alpha_{1}\right)^{1 / 2} C_{V}+2 C_{a}|\Omega|^{-1 / 2} ; C_{W}+|\Omega|^{-1 / s}\|Q\|_{L_{s}(\Omega)}+\lambda\right\}$. Below we will use (1.48) with $\boldsymbol{\Psi}=B^{0} \boldsymbol{\Phi}, \boldsymbol{\Phi} \in H^{4}\left(\mathcal{O} ; \mathbb{C}^{n}\right)$.

By analogy with (1.44), using (1.41) and (1.45) $-(1.47)$, we obtain

$$
\begin{aligned}
& \left\|\mathbf{D}^{2} B^{0} \boldsymbol{\Phi}\right\|_{L_{2}(\mathcal{O})} \leqslant\left\|\mathbf{D}^{2} b(\mathbf{D})^{*} g^{0} b(\mathbf{D}) \boldsymbol{\Phi}\right\|_{L_{2}(\mathcal{O})}+\left\|\mathbf{D}^{2} b(\mathbf{D})^{*} V \boldsymbol{\Phi}\right\|_{L_{2}(\mathcal{O})}+\left\|\mathbf{D}^{2} V^{*} b(\mathbf{D}) \boldsymbol{\Phi}\right\|_{L_{2}(\mathcal{O})} \\
& \quad+\sum_{j=1}^{d}\left\|\left(\overline{a_{j}+a_{j}^{*}}\right) \mathbf{D}^{2} D_{j} \boldsymbol{\Phi}\right\|_{L_{2}(\mathcal{O})}+\left\|\mathbf{D}^{2} W \boldsymbol{\Phi}\right\|_{L_{2}(\mathcal{O})}+\left\|\bar{Q} \mathbf{D}^{2} \boldsymbol{\Phi}\right\|_{L_{2}(\mathcal{O})}+\lambda\left\|\mathbf{D}^{2} \boldsymbol{\Phi}\right\|_{L_{2}(\mathcal{O})} \\
& \leqslant C_{B}\left(\left\|\mathbf{D}^{4} \boldsymbol{\Phi}\right\|_{L_{2}(\mathcal{O})}+\left\|\mathbf{D}^{3} \boldsymbol{\Phi}\right\|_{L_{2}(\mathcal{O})}+\left\|\mathbf{D}^{2} \boldsymbol{\Phi}\right\|_{L_{2}(\mathcal{O})}\right) .
\end{aligned}
$$

Similarly,

$$
\begin{aligned}
& \left\|\mathbf{D} B^{0} \boldsymbol{\Phi}\right\|_{L_{2}(\mathcal{O})} \leqslant C_{B}\left(\left\|\mathbf{D}^{3} \boldsymbol{\Phi}\right\|_{L_{2}(\mathcal{O})}+\left\|\mathbf{D}^{2} \boldsymbol{\Phi}\right\|_{L_{2}(\mathcal{O})}+\|\mathbf{D} \boldsymbol{\Phi}\|_{L_{2}(\mathcal{O})}\right), \\
& \left\|B^{0} \boldsymbol{\Phi}\right\|_{L_{2}(\mathcal{O})} \leqslant C_{B}\left(\left\|\mathbf{D}^{2} \boldsymbol{\Phi}\right\|_{L_{2}(\mathcal{O})}+\|\mathbf{D} \boldsymbol{\Phi}\|_{L_{2}(\mathcal{O})}+\|\boldsymbol{\Phi}\|_{L_{2}(\mathcal{O})}\right) .
\end{aligned}
$$

Combining (1.48)-(1.51), we have

$$
\begin{aligned}
& \left\|\left(B^{0}\right)^{2} \boldsymbol{\Phi}\right\|_{L_{2}(\mathcal{O})} \\
& \quad \leqslant C_{B}^{2}\left(\left\|\mathbf{D}^{4} \boldsymbol{\Phi}\right\|_{L_{2}(\mathcal{O})}+2\left\|\mathbf{D}^{3} \boldsymbol{\Phi}\right\|_{L_{2}(\mathcal{O})}+3\left\|\mathbf{D}^{2} \boldsymbol{\Phi}\right\|_{L_{2}(\mathcal{O})}+2\|\mathbf{D} \boldsymbol{\Phi}\|_{L_{2}(\mathcal{O})}+\|\boldsymbol{\Phi}\|_{L_{2}(\mathcal{O})}\right) \\
& \leqslant \sqrt{19} C_{B}^{2}\|\boldsymbol{\Phi}\|_{H^{4}(\mathcal{O})}, \quad \boldsymbol{\Phi} \in H^{4}\left(\mathcal{O} ; \mathbb{C}^{n}\right) .
\end{aligned}
$$

We arrive at estimate (1.43) with the constant $\mathfrak{C}:=\sqrt{19} C_{B}^{2}$.

1.7. Approximation of the resolvent $\left(B_{D, \varepsilon}-\zeta I\right)^{-1}$. Now we formulate the results of the paper [MSu3], where the behavior of the resolvent $\left(B_{D, \varepsilon}-\zeta I\right)^{-1}$ was studied. See also the brief communication [MSu4.

We choose the numbers $\varepsilon_{0}, \varepsilon_{1} \in(0,1]$ according to the following condition.

Condition 1.8. Let $\mathcal{O} \subset \mathbb{R}^{d}$ be a bounded domain. Denote

$$
(\partial \mathcal{O})_{\varepsilon}:=\left\{\mathbf{x} \in \mathbb{R}^{d}: \operatorname{dist}\{\mathbf{x} ; \partial \mathcal{O}\}<\varepsilon\right\} .
$$

Suppose that there exists a number $\varepsilon_{0} \in(0,1]$ such that the strip $(\partial \mathcal{O})_{\varepsilon_{0}}$ can be covered by a finite number of open sets admitting diffeomorphisms of class $C^{0,1}$ rectifying the boundary $\partial \mathcal{O}$. We set $\varepsilon_{1}:=\varepsilon_{0}\left(1+r_{1}\right)^{-1}$, where $2 r_{1}=\operatorname{diam} \Omega$. 
Obviously, the number $\varepsilon_{1}$ depends only on the domain $\mathcal{O}$ and the lattice $\Gamma$.

Note that Condition 1.8 is ensured only by the assumption that $\partial \mathcal{O}$ is Lipschitz; we imposed a more restrictive condition $\partial \mathcal{O} \in C^{1,1}$ in order to guarantee estimate (1.42).

The following result was obtained in [MSu3, Theorems 9.2 and 10.1].

Theorem 1.9. Let $\mathcal{O} \subset \mathbb{R}^{d}$ be a bounded domain of class $C^{1,1}$. Suppose that the assumptions of Subsec. 1.3 1.6] are satisfied. Suppose that $\varepsilon_{1}$ is subject to Condition 1.8 .

$1^{\circ}$. Let $\zeta=|\zeta| e^{i \phi} \in \mathbb{C} \backslash \mathbb{R}_{+},|\zeta| \geqslant 1$. Denote

$$
c(\phi):= \begin{cases}|\sin \phi|^{-1}, & \phi \in(0, \pi / 2) \cup(3 \pi / 2,2 \pi), \\ 1, & \phi \in[\pi / 2,3 \pi / 2] .\end{cases}
$$

Then for $0<\varepsilon \leqslant \varepsilon_{1}$ and $\zeta \in \mathbb{C} \backslash \mathbb{R}_{+},|\zeta| \geqslant 1$ we have

$$
\left\|\left(B_{D, \varepsilon}-\zeta I\right)^{-1}-\left(B_{D}^{0}-\zeta I\right)^{-1}\right\|_{L_{2}(\mathcal{O}) \rightarrow L_{2}(\mathcal{O})} \leqslant C_{1} c(\phi)^{2} \varepsilon|\zeta|^{-1 / 2} .
$$

$2^{\circ}$. Let $c_{b}$ be a common lower bound for the operators $B_{D}^{0}$ and $B_{D, \varepsilon}$ for $0<\varepsilon \leqslant \varepsilon_{1}$. Denote $\psi=\arg \left(\zeta-c_{b}\right), 0<\psi<2 \pi$, and

$$
\varrho_{b}(\zeta):= \begin{cases}c(\psi)^{2}\left|\zeta-c_{b}\right|^{-2}, & \left|\zeta-c_{b}\right|<1, \\ c(\psi)^{2}, & \left|\zeta-c_{b}\right| \geqslant 1 .\end{cases}
$$

Then for $0<\varepsilon \leqslant \varepsilon_{1}$ and $\zeta \in \mathbb{C} \backslash\left[c_{b}, \infty\right)$ we have

$$
\left\|\left(B_{D, \varepsilon}-\zeta I\right)^{-1}-\left(B_{D}^{0}-\zeta I\right)^{-1}\right\|_{L_{2}(\mathcal{O}) \rightarrow L_{2}(\mathcal{O})} \leqslant C_{2} \varrho_{b}(\zeta) \varepsilon .
$$

The constants $C_{1}$ and $C_{2}$ depend only on the problem data (1.7).

The constant $c_{b}$ in Theorem $1.9\left(2^{\circ}\right)$ is any common lower bound for the operators $B_{D}^{0}$ and $B_{D, \varepsilon}$. Taking into account inequalities (1.17), (1.38), and the expression for the constant $c_{*}$ (see (1.15)), we choose

$$
c_{b}:=4^{-1} \alpha_{0}\left\|g^{-1}\right\|_{L_{\infty}}^{-1}(\operatorname{diam} \mathcal{O})^{-2} .
$$

Fix a linear continuous extension operator

$$
P_{\mathcal{O}}: H^{l}\left(\mathcal{O} ; \mathbb{C}^{n}\right) \rightarrow H^{l}\left(\mathbb{R}^{d} ; \mathbb{C}^{n}\right), \quad l \in \mathbb{Z}_{+} .
$$

Such a ,,universal" extension operator exists for any Lipschitz bounded domain (see [St] or $[\mathrm{R}])$. We have

$$
\left\|P_{\mathcal{O}}\right\|_{H^{l}(\mathcal{O}) \rightarrow H^{l}\left(\mathbb{R}^{d}\right)} \leqslant C_{\mathcal{O}}^{(l)}, \quad l \in \mathbb{Z}_{+},
$$

where the constant $C_{\mathcal{O}}^{(l)}$ depends only on $l$ and the domain $\mathcal{O}$. Let $R_{\mathcal{O}}$ be the operator of restriction of functions in $\mathbb{R}^{d}$ to the domain $\mathcal{O}$. Denote

$$
K_{D}(\varepsilon ; \zeta):=R_{\mathcal{O}}\left(\left[\Lambda^{\varepsilon}\right] b(\mathbf{D})+\left[\widetilde{\Lambda}^{\varepsilon}\right]\right) S_{\varepsilon} P_{\mathcal{O}}\left(B_{D}^{0}-\zeta I\right)^{-1} .
$$

The corrector (1.58) is a continuous operator acting from $L_{2}\left(\mathcal{O} ; \mathbb{C}^{n}\right)$ to $H^{1}\left(\mathcal{O} ; \mathbb{C}^{n}\right)$. This can be checked by using Proposition 1.2 and inclusions $\Lambda, \widetilde{\Lambda} \in \widetilde{H}^{1}(\Omega)$. Note that $\left\|\varepsilon K_{D}(\varepsilon ; \zeta)\right\|_{L_{2}(\mathcal{O}) \rightarrow H^{1}(\mathcal{O})}=O(1)$ for small $\varepsilon$ and $\zeta$ fixed.

Let us formulate the results of [MSu3, Theorems 9.2 and 10.1].

Theorem 1.10. Under the assumptions of Theorem 1.9, let $K_{D}(\varepsilon ; \zeta)$ be the operator (1.58). $1^{\circ}$. For $0<\varepsilon \leqslant \varepsilon_{1}$ and $\zeta \in \mathbb{C} \backslash \mathbb{R}_{+},|\zeta| \geqslant 1$, we have

$$
\left\|\left(B_{D, \varepsilon}-\zeta I\right)^{-1}-\left(B_{D}^{0}-\zeta I\right)^{-1}-\varepsilon K_{D}(\varepsilon ; \zeta)\right\|_{L_{2}(\mathcal{O}) \rightarrow H^{1}(\mathcal{O})} \leqslant C_{3} c(\phi)^{2}\left(\varepsilon^{1 / 2}|\zeta|^{-1 / 4}+\varepsilon\right) .
$$

$2^{\circ}$. Let $c_{b}$ be the constant (1.55). Then for $0<\varepsilon \leqslant \varepsilon_{1}$ and $\zeta \in \mathbb{C} \backslash\left[c_{b}, \infty\right)$ we have

$$
\begin{aligned}
& \left\|\left(B_{D, \varepsilon}-\zeta I\right)^{-1}-\left(B_{D}^{0}-\zeta I\right)^{-1}-\varepsilon K_{D}(\varepsilon ; \zeta)\right\|_{L_{2}(\mathcal{O}) \rightarrow H^{1}(\mathcal{O})} \\
& \quad \leqslant C_{4}\left(\varepsilon^{1 / 2} \varrho_{b}(\zeta)^{1 / 2}+\varepsilon|1+\zeta|^{1 / 2} \varrho_{b}(\zeta)\right) .
\end{aligned}
$$

The constants $C_{3}$ and $C_{4}$ depend only on the problem data (1.7). 
Corollary 1.11. Under the assumptions of Theorem 1.10, for $0<\varepsilon \leqslant \varepsilon_{1}$ and $\zeta \in \mathbb{C} \backslash \mathbb{R}_{+},|\zeta| \geqslant 1$, we have

$$
\begin{aligned}
& \left\|\left(B_{D, \varepsilon}-\zeta I\right)^{-1}-\left(B_{D}^{0}-\zeta I\right)^{-1}-\varepsilon K_{D}(\varepsilon ; \zeta)\right\|_{L_{2}(\mathcal{O}) \rightarrow H^{1}(\mathcal{O})} \\
& \quad \leqslant C_{5}\left(c(\phi)^{2} \varepsilon^{1 / 2}|\zeta|^{-1 / 4}+c(\phi)^{3 / 2} \varepsilon^{1 / 2}\right) .
\end{aligned}
$$

The constant $C_{5}$ depends only on the problem data (1.7).

Corollary 1.11 follows from Theorem $1.10\left(1^{\circ}\right)$ and a rough estimate for the operators under the norm sign in (1.59). We need the estimates for the resolvents $\left(B_{D, \varepsilon}-\zeta I\right)^{-1}$ and $\left(B_{D}^{0}-\zeta I\right)^{-1}$ (see [MSu3, Lemmas 2.1 and 2.3]).

Lemma 1.12. For $0<\varepsilon \leqslant 1$ and $\zeta \in \mathbb{C} \backslash \mathbb{R}_{+}$we have

$$
\begin{aligned}
& \left\|\left(B_{D, \varepsilon}-\zeta I\right)^{-1}\right\|_{L_{2}(\mathcal{O}) \rightarrow L_{2}(\mathcal{O})} \leqslant c(\phi)|\zeta|^{-1}, \\
& \left\|\mathbf{D}\left(B_{D, \varepsilon}-\zeta I\right)^{-1}\right\|_{L_{2}(\mathcal{O}) \rightarrow L_{2}(\mathcal{O})} \leqslant \mathcal{C}_{4} c(\phi)|\zeta|^{-1 / 2}, \\
& \left\|\left(B_{D}^{0}-\zeta I\right)^{-1}\right\|_{L_{2}(\mathcal{O}) \rightarrow L_{2}(\mathcal{O})} \leqslant c(\phi)|\zeta|^{-1}, \\
& \left\|\mathbf{D}\left(B_{D}^{0}-\zeta I\right)^{-1}\right\|_{L_{2}(\mathcal{O}) \rightarrow L_{2}(\mathcal{O})} \leqslant \mathcal{C}_{4} c(\phi)|\zeta|^{-1 / 2}, \\
& \left\|\left(B_{D}^{0}-\zeta I\right)^{-1}\right\|_{L_{2}(\mathcal{O}) \rightarrow H^{2}(\mathcal{O})} \leqslant \mathcal{C}_{3} c(\phi) .
\end{aligned}
$$

Here $\mathcal{C}_{4}:=2^{3 / 2} \alpha_{0}^{-1 / 2}\left\|g^{-1}\right\|_{L_{\infty}}^{1 / 2}$ and the constant $\mathcal{C}_{3}$ is the same as in (1.42).

Proof of Corollary 1.11. Let us estimate the operator (1.58):

$$
\begin{aligned}
& \varepsilon\left\|K_{D}(\varepsilon ; \zeta)\right\|_{L_{2}(\mathcal{O}) \rightarrow H^{1}(\mathcal{O})} \\
& \quad \leqslant\left(\varepsilon\left\|\Lambda^{\varepsilon} S_{\varepsilon}\right\|_{L_{2}\left(\mathbb{R}^{d}\right) \rightarrow L_{2}\left(\mathbb{R}^{d}\right)}+\left\|(\mathbf{D} \Lambda)^{\varepsilon} S_{\varepsilon}\right\|_{L_{2}\left(\mathbb{R}^{d}\right) \rightarrow L_{2}\left(\mathbb{R}^{d}\right)}\right) \\
& \quad \times\left\|b(\mathbf{D}) P_{\mathcal{O}}\left(B_{D}^{0}-\zeta I\right)^{-1}\right\|_{L_{2}(\mathcal{O}) \rightarrow L_{2}\left(\mathbb{R}^{d}\right)} \\
& \quad+\left(\varepsilon\left\|\widetilde{\Lambda}^{\varepsilon} S_{\varepsilon}\right\|_{L_{2}\left(\mathbb{R}^{d}\right) \rightarrow L_{2}\left(\mathbb{R}^{d}\right)}+\left\|(\mathbf{D} \widetilde{\Lambda})^{\varepsilon} S_{\varepsilon}\right\|_{L_{2}\left(\mathbb{R}^{d}\right) \rightarrow L_{2}\left(\mathbb{R}^{d}\right)}\right) \\
& \quad \times\left\|P_{\mathcal{O}}\left(B_{D}^{0}-\zeta I\right)^{-1}\right\|_{L_{2}(\mathcal{O}) \rightarrow L_{2}\left(\mathbb{R}^{d}\right)} \\
& \quad+\varepsilon\left\|\Lambda^{\varepsilon} S_{\varepsilon}\right\|_{L_{2}\left(\mathbb{R}^{d}\right) \rightarrow L_{2}\left(\mathbb{R}^{d}\right)}\left\|\mathbf{D} b(\mathbf{D}) P_{\mathcal{O}}\left(B_{D}^{0}-\zeta I\right)^{-1}\right\|_{L_{2}(\mathcal{O}) \rightarrow L_{2}\left(\mathbb{R}^{d}\right)} \\
& \quad+\varepsilon\left\|\widetilde{\Lambda}^{\varepsilon} S_{\varepsilon}\right\|_{L_{2}\left(\mathbb{R}^{d}\right) \rightarrow L_{2}\left(\mathbb{R}^{d}\right)}\left\|\mathbf{D} P_{\mathcal{O}}\left(B_{D}^{0}-\zeta I\right)^{-1}\right\|_{L_{2}(\mathcal{O}) \rightarrow L_{2}\left(\mathbb{R}^{d}\right)} .
\end{aligned}
$$

By Proposition 1.2 and inequalities (1.25), (1.26), (1.33), and (1.34),

$$
\begin{aligned}
& \left\|\Lambda^{\varepsilon} S_{\varepsilon}\right\|_{L_{2}\left(\mathbb{R}^{d}\right) \rightarrow L_{2}\left(\mathbb{R}^{d}\right)} \leqslant M_{1}, \\
& \left\|(\mathbf{D} \Lambda)^{\varepsilon} S_{\varepsilon}\right\|_{L_{2}\left(\mathbb{R}^{d}\right) \rightarrow L_{2}\left(\mathbb{R}^{d}\right)} \leqslant M_{2}, \\
& \left\|\widetilde{\Lambda}^{\varepsilon} S_{\varepsilon}\right\|_{L_{2}\left(\mathbb{R}^{d}\right) \rightarrow L_{2}\left(\mathbb{R}^{d}\right)} \leqslant|\Omega|^{-1 / 2}\left(2 r_{0}\right)^{-1} C_{a} n^{1 / 2} \alpha_{0}^{-1}\left\|g^{-1}\right\|_{L_{\infty}}=: \widetilde{M}_{1}, \\
& \left\|(\mathbf{D} \widetilde{\Lambda})^{\varepsilon} S_{\varepsilon}\right\|_{L_{2}\left(\mathbb{R}^{d}\right) \rightarrow L_{2}\left(\mathbb{R}^{d}\right)} \leqslant|\Omega|^{-1 / 2} C_{a} n^{1 / 2} \alpha_{0}^{-1}\left\|g^{-1}\right\|_{L_{\infty}}=: \widetilde{M}_{2} .
\end{aligned}
$$

Combining these estimates, Lemma 1.12, and (1.2), (1.57), (1.62), for $0<\varepsilon \leqslant 1, \zeta \in \mathbb{C} \backslash \mathbb{R}_{+}$, $|\zeta| \geqslant 1$ we have

$$
\begin{aligned}
& \left\|\left(B_{D, \varepsilon}-\zeta I\right)^{-1}-\left(B_{D}^{0}-\zeta I\right)^{-1}-\varepsilon K_{D}(\varepsilon ; \zeta)\right\|_{L_{2}(\mathcal{O}) \rightarrow H^{1}(\mathcal{O})} \leqslant \widehat{C}_{5} c(\phi), \\
& \widehat{C}_{5}:=\left(2+\left(M_{1}+M_{2}\right) \alpha_{1}^{1 / 2} C_{\mathcal{O}}^{(1)}+\widetilde{M}_{1} C_{\mathcal{O}}^{(1)}\right)\left(1+\mathcal{C}_{4}\right)+\left(\widetilde{M}_{1}+\widetilde{M}_{2}\right) C_{\mathcal{O}}^{(0)}+M_{1} \alpha_{1}^{1 / 2} C_{\mathcal{O}}^{(2)} \mathcal{C}_{3} .
\end{aligned}
$$


Combining (1.59) and (1.65), for $0<\varepsilon \leqslant \varepsilon_{1}$ and $\zeta \in \mathbb{C} \backslash \mathbb{R}_{+},|\zeta| \geqslant 1$, we obtain

$$
\begin{aligned}
& \left\|\left(B_{D, \varepsilon}-\zeta I\right)^{-1}-\left(B_{D}^{0}-\zeta I\right)^{-1}-\varepsilon K_{D}(\varepsilon ; \zeta)\right\|_{L_{2}(\mathcal{O}) \rightarrow H^{1}(\mathcal{O})} \\
& \quad \leqslant \min \left\{C_{3} c(\phi)^{2}\left(\varepsilon^{1 / 2}|\zeta|^{-1 / 4}+\varepsilon\right) ; \widehat{C}_{5} c(\phi)\right\} \\
& \quad \leqslant C_{3} c(\phi)^{2} \varepsilon^{1 / 2}|\zeta|^{-1 / 4}+\min \left\{C_{3} c(\phi)^{2} \varepsilon ; \widehat{C}_{5} c(\phi)\right\} \\
& \quad \leqslant C_{3} c(\phi)^{2} \varepsilon^{1 / 2}|\zeta|^{-1 / 4}+\left(C_{3} \widehat{C}_{5}\right)^{1 / 2} c(\phi)^{3 / 2} \varepsilon^{1 / 2} .
\end{aligned}
$$

We arrive at estimate (1.61) with the constant $C_{5}:=\max \left\{C_{3} ;\left(C_{3} \widehat{C}_{5}\right)^{1 / 2}\right\}$.

1.8. Approximation of the operator $B_{D, \varepsilon}^{-1 / 2}$. The following result is a consequence of Theorem 1.9.

Lemma 1.13. Under the conditions of Theorem 1.9, for $0<\varepsilon \leqslant \varepsilon_{1}$ we have

$$
\left\|B_{D, \varepsilon}^{-1 / 2}-\left(B_{D}^{0}\right)^{-1 / 2}\right\|_{L_{2}(\mathcal{O}) \rightarrow L_{2}(\mathcal{O})} \leqslant C_{6} \varepsilon^{1 / 2} .
$$

The constant $C_{6}$ depends only on the problem data (1.7).

Proof. We have $B_{D, \varepsilon}^{-1 / 2}=\pi^{-1} \int_{0}^{\infty} \nu^{-1 / 2}\left(B_{D, \varepsilon}+\nu I\right)^{-1} d \nu$. See, e. g., ViGKo, Chapter III, $\S 3$, Subsec. 4]. For $\left(B_{D}^{0}\right)^{-1 / 2}$ we have the similar identity. Hence,

$$
\begin{aligned}
& \left\|B_{D, \varepsilon}^{-1 / 2}-\left(B_{D}^{0}\right)^{-1 / 2}\right\|_{L_{2}(\mathcal{O}) \rightarrow L_{2}(\mathcal{O})} \\
& \quad \leqslant \pi^{-1} \int_{0}^{\infty} \nu^{-1 / 2}\left\|\left(B_{D, \varepsilon}+\nu I\right)^{-1}-\left(B_{D}^{0}+\nu I\right)^{-1}\right\|_{L_{2}(\mathcal{O}) \rightarrow L_{2}(\mathcal{O})} d \nu .
\end{aligned}
$$

Since $c_{b}$ is a common lower bound for the operators $B_{D, \varepsilon}$ and $B_{D}^{0}$,

$$
\left\|\left(B_{D, \varepsilon}+\nu I\right)^{-1}-\left(B_{D}^{0}+\nu I\right)^{-1}\right\|_{L_{2}(\mathcal{O}) \rightarrow L_{2}(\mathcal{O})} \leqslant 2\left(\nu+c_{b}\right)^{-1}, \quad \nu \in \mathbb{R}_{+} .
$$

Thus,

$$
\begin{aligned}
& \left\|B_{D, \varepsilon}^{-1 / 2}-\left(B_{D}^{0}\right)^{-1 / 2}\right\|_{L_{2}(\mathcal{O}) \rightarrow L_{2}(\mathcal{O})} \\
& \quad \leqslant 2^{1 / 2} \pi^{-1} \int_{0}^{\infty} \nu^{-1 / 2}\left(\nu+c_{b}\right)^{-1 / 2}\left\|\left(B_{D, \varepsilon}+\nu I\right)^{-1}-\left(B_{D}^{0}+\nu I\right)^{-1}\right\|_{L_{2}(\mathcal{O}) \rightarrow L_{2}(\mathcal{O})}^{1 / 2} d \nu
\end{aligned}
$$

For $\nu \in[0,1]$, we use (1.54):

$$
\left\|\left(B_{D, \varepsilon}+\nu I\right)^{-1}-\left(B_{D}^{0}+\nu I\right)^{-1}\right\|_{L_{2}(\mathcal{O}) \rightarrow L_{2}(\mathcal{O})} \leqslant C_{2} \varepsilon \max \left\{1 ;\left(c_{b}+\nu\right)^{-2}\right\} \leqslant C_{2} \varepsilon \max \left\{1 ; c_{b}^{-2}\right\} .
$$

For $\nu>1$, we apply estimate (1.52):

$$
\left\|\left(B_{D, \varepsilon}+\nu I\right)^{-1}-\left(B_{D}^{0}+\nu I\right)^{-1}\right\|_{L_{2}(\mathcal{O}) \rightarrow L_{2}(\mathcal{O})} \leqslant C_{1} \varepsilon \nu^{-1 / 2}, \quad \nu>1 .
$$

It follows that

$$
\begin{aligned}
& \left\|B_{D, \varepsilon}^{-1 / 2}-\left(B_{D}^{0}\right)^{-1 / 2}\right\|_{L_{2}(\mathcal{O}) \rightarrow L_{2}(\mathcal{O})} \\
& \leqslant 2^{1 / 2} \pi^{-1} C_{2}^{1 / 2} \max \left\{1 ; c_{b}^{-1}\right\} \varepsilon^{1 / 2} \int_{0}^{1} \nu^{-1 / 2}\left(\nu+c_{b}\right)^{-1 / 2} d \nu \\
& +2^{1 / 2} \pi^{-1} C_{1}^{1 / 2} \varepsilon^{1 / 2} \int_{1}^{\infty} \nu^{-1 / 2}\left(\nu+c_{b}\right)^{-1 / 2} \nu^{-1 / 4} d \nu .
\end{aligned}
$$

Evaluating these integrals, we arrive at estimate (1.66) with the constant

$$
C_{6}:=2^{3 / 2} \pi^{-1} C_{2}^{1 / 2} c_{b}^{-1 / 2} \max \left\{1 ; c_{b}^{-1}\right\}+2^{5 / 2} \pi^{-1} C_{1}^{1 / 2} .
$$




\section{Problem setting. Main Results}

2.1. The first initial-boundary value problem for hyperbolic systems. Our goal is to study the behavior of the solution of the following problem for small $\varepsilon$ :

$$
\left\{\begin{array}{l}
\frac{\partial^{2} \mathbf{u}_{\varepsilon}}{\partial t^{2}}(\mathbf{x}, t)=-\left(B_{\varepsilon} \mathbf{u}_{\varepsilon}\right)(\mathbf{x}, t)+\mathbf{F}(\mathbf{x}, t) \\
\left.\mathbf{u}_{\varepsilon}(\cdot, t)\right|_{\partial \mathcal{O}}=0, \\
\mathbf{u}_{\varepsilon}(\mathbf{x}, 0)=\boldsymbol{\varphi}(\mathbf{x}), \quad \frac{\partial \mathbf{u}_{\varepsilon}}{\partial t}(\mathbf{x}, 0)=\boldsymbol{\psi}(\mathbf{x})
\end{array}\right.
$$

Here $\boldsymbol{\varphi}, \boldsymbol{\psi} \in \operatorname{Dom}\left(B_{D}^{0}\right)^{2}, \mathbf{F} \in L_{1, \text { loc }}\left(\mathbb{R} ; \operatorname{Dom}\left(B_{D}^{0}\right)^{2}\right)$. (The imposed restrictions are caused by the technique used in the present work.) We have

$$
\mathbf{u}_{\varepsilon}(\cdot, t)=\cos \left(t B_{D, \varepsilon}^{1 / 2}\right) \boldsymbol{\varphi}+B_{D, \varepsilon}^{-1 / 2} \sin \left(t B_{D, \varepsilon}^{1 / 2}\right) \boldsymbol{\psi}+\int_{0}^{t} B_{D, \varepsilon}^{-1 / 2} \sin \left((t-\widetilde{t}) B_{D, \varepsilon}^{1 / 2}\right) \mathbf{F}(\cdot, \widetilde{t}) d \widetilde{t}
$$

So, to study the behavior of $\mathbf{u}_{\varepsilon}(\cdot, t)$ it suffices to obtain approximations for the operators $\cos \left(t B_{D, \varepsilon}^{1 / 2}\right)$ and $B_{D, \varepsilon}^{-1 / 2} \sin \left(t B_{D, \varepsilon}^{1 / 2}\right)$.

The effective problem is given by

$$
\left\{\begin{array}{l}
\frac{\partial^{2} \mathbf{u}_{0}}{\partial t^{2}}(\mathbf{x}, t)=-\left(B^{0} \mathbf{u}_{0}\right)(\mathbf{x}, t)+\mathbf{F}(\mathbf{x}, t) \\
\left.\mathbf{u}_{0}(\cdot, t)\right|_{\partial \mathcal{O}}=0, \\
\mathbf{u}_{0}(\mathbf{x}, 0)=\boldsymbol{\varphi}(\mathbf{x}), \quad \frac{\partial \mathbf{u}_{0}}{\partial t}(\mathbf{x}, 0)=\boldsymbol{\psi}(\mathbf{x})
\end{array}\right.
$$

Then

$$
\begin{aligned}
\mathbf{u}_{0}(\cdot, t) & =\cos \left(t\left(B_{D}^{0}\right)^{1 / 2}\right) \boldsymbol{\varphi}+\left(B_{D}^{0}\right)^{-1 / 2} \sin \left(t\left(B_{D}^{0}\right)^{1 / 2}\right) \boldsymbol{\psi} \\
& +\int_{0}^{t}\left(B_{D}^{0}\right)^{-1 / 2} \sin \left((t-\widetilde{t})\left(B_{D}^{0}\right)^{1 / 2}\right) \mathbf{F}(\cdot, \widetilde{t}) \widetilde{t}
\end{aligned}
$$

\subsection{Main results in the operator terms.}

Theorem 2.1. Let $\mathcal{O} \subset \mathbb{R}^{d}$ be a bounded domain of class $C^{1,1}$. Suppose that the assumptions of Subsec. 1.31.6 are satisfied. Let $\varepsilon_{1}$ be subject to Condition 1.8 . Then for $t \in \mathbb{R}$ and $0<\varepsilon \leqslant \varepsilon_{1}$ we have

$$
\begin{aligned}
& \left\|\left(\cos \left(t B_{D, \varepsilon}^{1 / 2}\right)-\cos \left(t\left(B_{D}^{0}\right)^{1 / 2}\right)\right)\left(B_{D}^{0}\right)^{-2}\right\|_{L_{2}(\mathcal{O}) \rightarrow L_{2}(\mathcal{O})} \leqslant C_{7} \varepsilon\left(1+|t|^{5}\right) \\
& \left\|\left(B_{D, \varepsilon}^{-1 / 2} \sin \left(t B_{D, \varepsilon}^{1 / 2}\right)-\left(B_{D}^{0}\right)^{-1 / 2} \sin \left(t\left(B_{D}^{0}\right)^{1 / 2}\right)\right)\left(B_{D}^{0}\right)^{-2}\right\|_{L_{2}(\mathcal{O}) \rightarrow L_{2}(\mathcal{O})} \leqslant C_{7} \varepsilon|t|\left(1+|t|^{5}\right) .
\end{aligned}
$$

The constant $C_{7}$ depends only on the problem data (1.7).

It seems natural to expect that, for hyperbolic systems, the analog of Theorem 1.10 holds true. However, according to the results of [BrOtFMu], it is impossible to approximate the operator $\cos \left(t B_{D, \varepsilon}^{1 / 2}\right)$ in the energy norm, while the operators $B_{D, \varepsilon}^{-1} \cos \left(t B_{D, \varepsilon}^{1 / 2}\right)$ and $B_{D, \varepsilon}^{-1 / 2} \sin \left(t B_{D, \varepsilon}^{1 / 2}\right)$ can be approximated (see Theorems 2.2 and 2.3 below). We also approximate the operator $g^{\varepsilon} b(\mathbf{D}) B_{D, \varepsilon}^{-1 / 2} \sin \left(t B_{D, \varepsilon}^{1 / 2}\right)$ which corresponds to the ,flux."

Theorem 2.2. Suppose that the assumptions of Theorem 2.1 are satisfied. Let matrixvalued functions $\Lambda(\mathbf{x})$ and $\widetilde{\Lambda}(\mathbf{x})$ be the $\Gamma$-periodic solutions of problems (1.21) and (1.31), respectively. Let $S_{\varepsilon}$ be the Steklov smoothing operator (1.1) and let $P_{\mathcal{O}}$ be the linear extension operator (1.56). Then for $t \in \mathbb{R}$ and $0<\varepsilon \leqslant \varepsilon_{1}$ we have

$$
\begin{aligned}
& \|\left(B_{D, \varepsilon}^{-1 / 2} \sin \left(t B_{D, \varepsilon}^{1 / 2}\right)-\left(B_{D}^{0}\right)^{-1 / 2} \sin \left(t\left(B_{D}^{0}\right)^{1 / 2}\right)\right. \\
& \left.\quad-\varepsilon\left(\Lambda^{\varepsilon} b(\mathbf{D})+\widetilde{\Lambda}^{\varepsilon}\right) S_{\varepsilon} P_{\mathcal{O}}\left(B_{D}^{0}\right)^{-1 / 2} \sin \left(t\left(B_{D}^{0}\right)^{1 / 2}\right)\right)\left(B_{D}^{0}\right)^{-2} \|_{L_{2}(\mathcal{O}) \rightarrow H^{1}(\mathcal{O})} \\
& \leqslant C_{8} \varepsilon^{1 / 2}\left(1+t^{6}\right) .
\end{aligned}
$$


Let $\widetilde{g}(\mathbf{x})$ be the matrix-valued function (1.22). Denote

$$
G_{D}(\varepsilon ; t):=\left(\widetilde{g}^{\varepsilon} S_{\varepsilon} b(\mathbf{D})+g^{\varepsilon}(b(\mathbf{D}) \widetilde{\Lambda})^{\varepsilon} S_{\varepsilon}\right) P_{\mathcal{O}}\left(B_{D}^{0}\right)^{-1 / 2} \sin \left(t\left(B_{D}^{0}\right)^{1 / 2}\right) .
$$

Then for $t \in \mathbb{R}$ and $0<\varepsilon \leqslant \varepsilon_{1}$ we have

$$
\left\|\left(g^{\varepsilon} b(\mathbf{D}) B_{D, \varepsilon}^{-1 / 2} \sin \left(t B_{D, \varepsilon}^{1 / 2}\right)-G_{D}(\varepsilon ; t)\right)\left(B_{D}^{0}\right)^{-2}\right\|_{L_{2}(\mathcal{O}) \rightarrow L_{2}(\mathcal{O})} \leqslant C_{9} \varepsilon^{1 / 2}\left(1+t^{6}\right) .
$$

Here the constants $C_{8}$ and $C_{9}$ depend only on the problem data (1.7).

The proofs of Theorems 2.1 and 2.2 are given below in Section 3 .

\subsection{On approximation of the operator $\cos \left(t B_{D, \varepsilon}^{1 / 2}\right) B_{D, \varepsilon}^{-1}$ in the energy norm.}

Theorem 2.3. Under the assumptions of Theorem $\left[2.2\right.$, for $t \in \mathbb{R} \backslash\{0\}$ and $0<\varepsilon \leqslant \varepsilon_{1}$ we have

$$
\begin{aligned}
& \|\left(\cos \left(t B_{D, \varepsilon}^{1 / 2}\right) B_{D, \varepsilon}^{-1}-\cos \left(t\left(B_{D}^{0}\right)^{1 / 2}\right)\left(B_{D}^{0}\right)^{-1}\right. \\
& \left.\quad-\varepsilon\left(\Lambda^{\varepsilon} b(\mathbf{D})+\widetilde{\Lambda}^{\varepsilon}\right) S_{\varepsilon} P_{\mathcal{O}} \cos \left(t\left(B_{D}^{0}\right)^{1 / 2}\right)\left(B_{D}^{0}\right)^{-1}\right)\left(B_{D}^{0}\right)^{-1} \|_{L_{2}(\mathcal{O}) \rightarrow H^{1}(\mathcal{O})} \\
& \leqslant C_{10} \varepsilon^{1 / 2}\left(1+|t|^{5}\right)
\end{aligned}
$$

The constant $C_{10}$ depends only on the problem data (1.7).

The proof of Theorem 2.3 is given below in Section 3 .

Theorem 2.3 allows us to obtain approximation in the energy class for the solution of the hyperbolic problem with the special choice of the initial data:

$$
\partial_{t}^{2} \mathbf{u}_{\varepsilon}=-B_{\varepsilon} \mathbf{u}_{\varepsilon},\left.\quad \mathbf{u}_{\varepsilon}(\cdot, t)\right|_{\partial \mathcal{O}}=0,\left.\quad \mathbf{u}_{\varepsilon}\right|_{t=0}=B_{D, \varepsilon}^{-1} \boldsymbol{\varphi},\left.\quad\left(\partial_{t} \mathbf{u}_{\varepsilon}\right)\right|_{t=0}=0 .
$$

Here $\varphi \in \operatorname{Dom} B_{D}^{0}=H^{2}\left(\mathcal{O} ; \mathbb{C}^{n}\right) \cap H_{0}^{1}\left(\mathcal{O} ; \mathbb{C}^{n}\right)$. In this case, the effective problem has the form

$$
\partial_{t}^{2} \mathbf{u}_{0}=-B^{0} \mathbf{u}_{0},\left.\quad \mathbf{u}_{0}(\cdot, t)\right|_{\partial \mathcal{O}}=0,\left.\quad \mathbf{u}_{0}\right|_{t=0}=\left(B_{D}^{0}\right)^{-1} \boldsymbol{\varphi},\left.\quad\left(\partial_{t} \mathbf{u}_{0}\right)\right|_{t=0}=0 .
$$

From (1.48) and (2.9) it follows that

$$
\left\|\mathbf{u}_{\varepsilon}(\cdot, t)-\mathbf{u}_{0}(\cdot, t)-\varepsilon\left(\Lambda^{\varepsilon} b(\mathbf{D})+\widetilde{\Lambda}^{\varepsilon}\right) S_{\varepsilon} \widetilde{\mathbf{u}}_{0}(\cdot, t)\right\|_{H^{1}(\mathcal{O})} \leqslant 3^{1 / 2} C_{B} C_{10} \varepsilon^{1 / 2}\left(1+|t|^{5}\right)\|\boldsymbol{\varphi}\|_{H^{2}(\mathcal{O})}
$$

For such choice of the initial data, the possibility to approximate the solution in the energy class is in accordance with the results of [BrOtFMu].

Note that Lemma 1.13, Theorem 2.3, and estimates (1.18), (1.40) allow us to approximate the operator $\cos \left(t B_{D, \varepsilon}^{1 / 2}\right) B_{D, \varepsilon}^{-1 / 2}$. We have

$$
\begin{aligned}
& \left\|\cos \left(t B_{D, \varepsilon}^{1 / 2}\right) B_{D, \varepsilon}^{-1 / 2}\left(B_{D, \varepsilon}^{-1 / 2}-\left(B_{D}^{0}\right)^{-1 / 2}\right)\left(B_{D}^{0}\right)^{-1}\right\|_{L_{2}(\mathcal{O}) \rightarrow H^{1}(\mathcal{O})} \\
& \quad \leqslant c_{3}\left\|B_{D, \varepsilon}^{-1 / 2}-\left(B_{D}^{0}\right)^{-1 / 2}\right\|_{L_{2}(\mathcal{O}) \rightarrow L_{2}(\mathcal{O})}\left\|\left(B_{D}^{0}\right)^{-1}\right\|_{L_{2}(\mathcal{O}) \rightarrow L_{2}(\mathcal{O})} \leqslant c_{3} C_{6} \mathcal{C}_{1} \varepsilon^{1 / 2}
\end{aligned}
$$

Combining this with (2.9), we obtain

$$
\begin{aligned}
& \|\left(\cos \left(t B_{D, \varepsilon}^{1 / 2}\right) B_{D, \varepsilon}^{-1 / 2}-\left(I+\varepsilon\left(\Lambda^{\varepsilon} b(\mathbf{D})+\widetilde{\Lambda}^{\varepsilon}\right) S_{\varepsilon} P_{\mathcal{O}}\right) \cos \left(t\left(B_{D}^{0}\right)^{1 / 2}\right)\left(B_{D}^{0}\right)^{-1 / 2}\right) \\
& \quad \times\left(B_{D}^{0}\right)^{-3 / 2} \|_{L_{2}(\mathcal{O}) \rightarrow H^{1}(\mathcal{O})} \leqslant\left(C_{10}+c_{3} C_{6} \mathcal{C}_{1}\right) \varepsilon^{1 / 2}\left(1+|t|^{5}\right) .
\end{aligned}
$$


2.4. Removal of the smoothing operator from the corrector. It turns out that the smoothing operator can be removed from the corrector if the matrix-valued functions $\Lambda(\mathbf{x})$ and $\widetilde{\Lambda}(\mathbf{x})$ are subject to some additional assumptions.

Condition 2.4. Assume that the $\Gamma$-periodic solution $\Lambda(\mathbf{x})$ of problem (1.21) is bounded, i. e., $\Lambda \in L_{\infty}\left(\mathbb{R}^{d}\right)$.

Some cases where Condition 2.4 is fulfilled automatically were distinguished in $\overline{B S u}$, Lemma 8.7].

Proposition 2.5. Suppose that at least one of the following assumptions is satisfied:

$\left.1^{\circ}\right) d \leqslant 2$

$2^{\circ}$ ) the dimension $d \geqslant 1$ is arbitrary, and the differential expression $A_{\varepsilon}$ is given by $A_{\varepsilon}=$ $\mathbf{D}^{*} g^{\varepsilon}(\mathbf{x}) \mathbf{D}$, where $g(\mathbf{x})$ is a symmetric matrix with real entries;

$3^{\circ}$ ) the dimension $d$ is arbitrary, and $g^{0}=\underline{g}$, i. e., relations (1.30) are satisfied.

Then Condition 2.4 holds.

In order to remove $S_{\varepsilon}$ from the term involving $\widetilde{\Lambda}^{\varepsilon}$, it suffices to impose the following condition.

Condition 2.6. Assume that the $\Gamma$-periodic solution $\widetilde{\Lambda}(\mathbf{x})$ of problem (1.31) is such that

$$
\widetilde{\Lambda} \in L_{p}(\Omega), \quad p=2 \text { for } d=1, \quad p>2 \text { for } d=2, \quad p=d \text { for } d \geqslant 3 .
$$

The following result was obtained in [Su3, Proposition 8.11].

Proposition 2.7. Condition 2.6 is fulfilled, if at least one of the following assumptions is satisfied:

$\left.1^{\circ}\right) d \leqslant 4$

$2^{\circ}$ ) the dimension $d$ is arbitrary, and the differential expression $A_{\varepsilon}$ has the form $A_{\varepsilon}=\mathbf{D}^{*} g^{\varepsilon}(\mathbf{x}) \mathbf{D}$, where $g(\mathbf{x})$ is a symmetric matrix-valued function with real entries.

Remark 2.8. If $A_{\varepsilon}=\mathbf{D}^{*} g^{\varepsilon}(\mathbf{x}) \mathbf{D}$, where $g(\mathbf{x})$ is a symmetric matrix-valued function with real entries, from [LaU, Chapter III, Theorem 13.1] it follows that $\Lambda, \widetilde{\Lambda} \in L_{\infty}$ and the norm $\|\Lambda\|_{L_{\infty}}$ is controlled in terms of $d,\|g\|_{L_{\infty}},\left\|g^{-1}\right\|_{L_{\infty}}$, and $\Omega$; the norm $\|\widetilde{\Lambda}\|_{L_{\infty}}$ does not exceed a constant depending on $d, \rho,\|g\|_{L_{\infty}},\left\|g^{-1}\right\|_{L_{\infty}},\left\|a_{j}\right\|_{L_{\rho}(\Omega)}, j=1, \ldots, d$, and $\Omega$. In this case, Conditions 2.4 and 2.6 are fulfilled simultaneously.

In this subsection, our goal is to prove the following statement.

Theorem 2.9. Suppose that the assumptions of Theorem 2.2 are satisfied. Assume that the matrix-valued function $\Lambda(\mathbf{x})$ is subject to Condition 2.4 and the matrix-valued function $\widetilde{\Lambda}(\mathbf{x})$ satisfies Condition 2.6. Denote

$$
G_{D}^{0}(\varepsilon ; t):=\left(\widetilde{g}^{\varepsilon} b(\mathbf{D})+g^{\varepsilon}(b(\mathbf{D}) \widetilde{\Lambda})^{\varepsilon}\right)\left(B_{D}^{0}\right)^{-1 / 2} \sin \left(t\left(B_{D}^{0}\right)^{1 / 2}\right) .
$$

Then for $t \in \mathbb{R}$ and $0<\varepsilon \leqslant \varepsilon_{1}$ we have

$$
\begin{aligned}
& \|\left(B_{D, \varepsilon}^{-1 / 2} \sin \left(t B_{D, \varepsilon}^{1 / 2}\right)-\left(I+\varepsilon \Lambda^{\varepsilon} b(\mathbf{D})+\varepsilon \widetilde{\Lambda}^{\varepsilon}\right)\left(B_{D}^{0}\right)^{-1 / 2} \sin \left(t\left(B_{D}^{0}\right)^{1 / 2}\right)\right) \\
& \quad \times\left(B_{D}^{0}\right)^{-2} \|_{L_{2}(\mathcal{O}) \rightarrow H^{1}(\mathcal{O})} \leqslant C_{11} \varepsilon^{1 / 2}\left(1+t^{6}\right), \\
& \left\|\left(g^{\varepsilon} b(\mathbf{D}) B_{D, \varepsilon}^{-1 / 2} \sin \left(t B_{D, \varepsilon}^{1 / 2}\right)-G_{D}^{0}(\varepsilon ; t)\right)\left(B_{D}^{0}\right)^{-2}\right\|_{L_{2}(\mathcal{O}) \rightarrow L_{2}(\mathcal{O})} \leqslant C_{12} \varepsilon^{1 / 2}\left(1+t^{6}\right) .
\end{aligned}
$$

The constants $C_{11}$ and $C_{12}$ depend only on the problem data (1.7), on $p$, and on the norms $\|\Lambda\|_{L_{\infty}},\|\widetilde{\Lambda}\|_{L_{p}(\Omega)}$.

To prove Theorem 2.9, we need the following results obtained in [MSu3, Lemmas 7.7 and $7.8]$. 
Lemma 2.10. Let $\Gamma$-periodic matrix-valued solution $\Lambda(\mathbf{x})$ of problem (1.21) satisfy Condition 2.4. Let $S_{\varepsilon}$ be the Steklov smoothing operator (1.1). Then for $0<\varepsilon \leqslant 1$

$$
\left\|\left[\Lambda^{\varepsilon}\right] b(\mathbf{D})\left(S_{\varepsilon}-I\right)\right\|_{H^{2}\left(\mathbb{R}^{d}\right) \rightarrow H^{1}\left(\mathbb{R}^{d}\right)} \leqslant \mathfrak{C}_{\Lambda} .
$$

The constant $\mathfrak{C}_{\Lambda}$ depends only on $m, d, \alpha_{0}, \alpha_{1},\|g\|_{L_{\infty}},\left\|g^{-1}\right\|_{L_{\infty}}$, on the parameters of the lattice $\Gamma$, and on the norm $\|\Lambda\|_{L_{\infty}}$.

Lemma 2.11. Let matrix-valued $\Gamma$-periodic solution $\widetilde{\Lambda}(\mathbf{x})$ of problem (1.31) satisfy Condition 2.6. Let $S_{\varepsilon}$ be the Steklov smoothing operator (1.1). Then for $0<\varepsilon \leqslant 1$

$$
\left\|\left[\widetilde{\Lambda}^{\varepsilon}\right]\left(S_{\varepsilon}-I\right)\right\|_{H^{2}\left(\mathbb{R}^{d}\right) \rightarrow H^{1}\left(\mathbb{R}^{d}\right)} \leqslant \mathfrak{C}_{\widetilde{\Lambda}} .
$$

The constant $\mathfrak{C}_{\widetilde{\Lambda}}$ is controlled in terms of $n, d, \alpha_{0}, \alpha_{1}, \rho,\|g\|_{L_{\infty}},\left\|g^{-1}\right\|_{L_{\infty}}$, the norms $\left\|a_{j}\right\|_{L_{\rho}(\Omega)}, j=1, \ldots, d, p,\|\widetilde{\Lambda}\|_{L_{p}(\Omega)}$, and the parameters of the lattice $\Gamma$.

The following assertion can be easily checked by using the Hölder inequality and the Sobolev embedding theorem (cf. [MSu1, Lemma 3.5]).

Lemma 2.12. Assume that the matrix-valued function $\widetilde{\Lambda}(\mathbf{x})$ satisfies Condition 2.6 . Then for $0<\varepsilon \leqslant 1$ the operator $\left[\widetilde{\Lambda}^{\varepsilon}\right]$ is a continuous mapping from $H^{1}\left(\mathbb{R}^{d} ; \mathbb{C}^{n}\right)$ to $L_{2}\left(\mathbb{R}^{d} ; \mathbb{C}^{n}\right)$ and

$$
\left\|\left[\widetilde{\Lambda}^{\varepsilon}\right]\right\|_{H^{1}\left(\mathbb{R}^{d}\right) \rightarrow L_{2}\left(\mathbb{R}^{d}\right)} \leqslant\|\widetilde{\Lambda}\|_{L_{p}(\Omega)} C_{\Omega}(p),
$$

where $C_{\Omega}(p)$ is the norm of the embedding operator $H^{1}(\Omega) \hookrightarrow L_{2(p / 2)^{\prime}}(\Omega)$. Here $(p / 2)^{\prime}=\infty$ for $d=1$ and $(p / 2)^{\prime}=p /(p-2)$ for $d \geqslant 2$.

Proof of Theorem 2.9. The result of Theorem 2.9 can be derived from Theorem 2.2 with the help of Lemmas 2.10, 2.11, and 2.12.

By Lemma 2.10 and (1.57),

$$
\begin{aligned}
& \left\|\Lambda^{\varepsilon} b(\mathbf{D})\left(S_{\varepsilon}-I\right) P_{\mathcal{O}}\left(B_{D}^{0}\right)^{-5 / 2} \sin \left(t\left(B_{D}^{0}\right)^{1 / 2}\right)\right\|_{L_{2}(\mathcal{O}) \rightarrow H^{1}\left(\mathbb{R}^{d}\right)} \\
& \quad \leqslant \mathfrak{C}_{\Lambda} C_{\mathcal{O}}^{(2)}\left\|\left(B_{D}^{0}\right)^{-5 / 2} \sin \left(t\left(B_{D}^{0}\right)^{1 / 2}\right)\right\|_{L_{2}(\mathcal{O}) \rightarrow H^{2}(\mathcal{O})}, \quad t \in \mathbb{R}, \quad 0<\varepsilon \leqslant 1 .
\end{aligned}
$$

We have

$$
\begin{aligned}
& \left\|\left(B_{D}^{0}\right)^{-5 / 2} \sin \left(t\left(B_{D}^{0}\right)^{1 / 2}\right)\right\|_{L_{2}(\mathcal{O}) \rightarrow H^{2}(\mathcal{O})} \\
& \quad \leqslant\left\|\left(B_{D}^{0}\right)^{-1}\right\|_{L_{2}(\mathcal{O}) \rightarrow H^{2}(\mathcal{O})}\left\|\left(B_{D}^{0}\right)^{-1}\right\|_{L_{2}(\mathcal{O}) \rightarrow L_{2}(\mathcal{O})}\left\|\left(B_{D}^{0}\right)^{-1 / 2} \sin \left(t\left(B_{D}^{0}\right)^{1 / 2}\right)\right\|_{L_{2}(\mathcal{O}) \rightarrow L_{2}(\mathcal{O})} .
\end{aligned}
$$

By the spectral theorem and the elementary inequality $|\sin \mu| /|\mu| \leqslant 1, \mu \in \mathbb{R}$,

$$
\left\|\left(B_{D}^{0}\right)^{-1 / 2} \sin \left(t\left(B_{D}^{0}\right)^{1 / 2}\right)\right\|_{L_{2}(\mathcal{O}) \rightarrow L_{2}(\mathcal{O})} \leqslant|t| .
$$

Combining this with (1.40), (1.42), and (2.14), we obtain

$$
\left\|\left(B_{D}^{0}\right)^{-5 / 2} \sin \left(t\left(B_{D}^{0}\right)^{1 / 2}\right)\right\|_{L_{2}(\mathcal{O}) \rightarrow H^{2}(\mathcal{O})} \leqslant \mathcal{C}_{1} \mathcal{C}_{3}|t|, \quad t \in \mathbb{R} .
$$

From Lemma 2.11 and (1.57), (2.15) it follows that

$$
\begin{aligned}
& \left\|\widetilde{\Lambda}^{\varepsilon}\left(S_{\varepsilon}-I\right) P_{\mathcal{O}}\left(B_{D}^{0}\right)^{-5 / 2} \sin \left(t\left(B_{D}^{0}\right)^{1 / 2}\right)\right\|_{L_{2}(\mathcal{O}) \rightarrow H^{1}\left(\mathbb{R}^{d}\right)} \\
& \quad \leqslant \mathfrak{C}_{\widetilde{\Lambda}} C_{\mathcal{O}}^{(2)}\left\|\left(B_{D}^{0}\right)^{-5 / 2} \sin \left(t\left(B_{D}^{0}\right)^{1 / 2}\right)\right\|_{L_{2}(\mathcal{O}) \rightarrow H^{2}(\mathcal{O})} \\
& \quad \leqslant \mathfrak{C}_{\widetilde{\Lambda}} C_{\mathcal{O}}^{(2)} \mathcal{C}_{1} \mathcal{C}_{3}|t|, \quad t \in \mathbb{R}, \quad 0<\varepsilon \leqslant 1 .
\end{aligned}
$$

Bringing together (2.7), (2.13), (2.15), and (2.16), we arrive at estimate (2.11) with the constant $C_{11}:=C_{8}+\left(\mathfrak{C}_{\Lambda}+\mathfrak{C}_{\widetilde{\Lambda}}\right) C_{\mathcal{O}}^{(2)} \mathcal{C}_{1} \mathcal{C}_{3}$. Here the inequality $|t| \leqslant\left(1+t^{6}\right), t \in \mathbb{R}$, is taken into account.

Now we proceed to the proof of inequality (2.12). By (1.3) and (2.11),

$$
\begin{aligned}
& \|\left(g^{\varepsilon} b(\mathbf{D}) B_{D, \varepsilon}^{-1 / 2} \sin \left(t B_{D, \varepsilon}^{1 / 2}\right)-g^{\varepsilon} b(\mathbf{D})\left(I+\varepsilon \Lambda^{\varepsilon} b(\mathbf{D})+\varepsilon \widetilde{\Lambda}^{\varepsilon}\right)\left(B_{D}^{0}\right)^{-1 / 2} \sin \left(t\left(B_{D}^{0}\right)^{1 / 2}\right)\right) \\
& \quad \times\left(B_{D}^{0}\right)^{-2}\left\|_{L_{2}(\mathcal{O}) \rightarrow L_{2}(\mathcal{O})} \leqslant\left(d \alpha_{1}\right)^{1 / 2}\right\| g \|_{L_{\infty}} C_{11} \varepsilon^{1 / 2}\left(1+t^{6}\right), \quad t \in \mathbb{R}, \quad 0<\varepsilon \leqslant \varepsilon_{1} .
\end{aligned}
$$


We have

$$
\begin{aligned}
& g^{\varepsilon} b(\mathbf{D})\left(I+\varepsilon \Lambda^{\varepsilon} b(\mathbf{D})+\varepsilon \widetilde{\Lambda}^{\varepsilon}\right)\left(B_{D}^{0}\right)^{-5 / 2} \sin \left(t\left(B_{D}^{0}\right)^{1 / 2}\right) \\
& \quad=g^{\varepsilon} b(\mathbf{D})\left(B_{D}^{0}\right)^{-5 / 2} \sin \left(t\left(B_{D}^{0}\right)^{1 / 2}\right)+g^{\varepsilon}(b(\mathbf{D}) \Lambda)^{\varepsilon} b(\mathbf{D})\left(B_{D}^{0}\right)^{-5 / 2} \sin \left(t\left(B_{D}^{0}\right)^{1 / 2}\right) \\
& \quad+g^{\varepsilon}(b(\mathbf{D}) \widetilde{\Lambda})^{\varepsilon}\left(B_{D}^{0}\right)^{-5 / 2} \sin \left(t\left(B_{D}^{0}\right)^{1 / 2}\right) \\
& \quad+\varepsilon \sum_{l=1}^{d} g^{\varepsilon} b_{l}\left(\Lambda^{\varepsilon} b(\mathbf{D})+\widetilde{\Lambda}^{\varepsilon}\right) D_{l}\left(B_{D}^{0}\right)^{-5 / 2} \sin \left(t\left(B_{D}^{0}\right)^{1 / 2}\right) .
\end{aligned}
$$

To estimate the fourth term in the right-hand side of (2.18), we use Conditions 2.4 and 2.6, Lemma 2.12, and inequality (1.3):

$$
\begin{aligned}
& \left\|\varepsilon \sum_{l=1}^{d} g^{\varepsilon} b_{l}\left(\Lambda^{\varepsilon} b(\mathbf{D})+\widetilde{\Lambda}^{\varepsilon}\right) D_{l}\left(B_{D}^{0}\right)^{-5 / 2} \sin \left(t\left(B_{D}^{0}\right)^{1 / 2}\right)\right\|_{L_{2}(\mathcal{O}) \rightarrow L_{2}(\mathcal{O})} \\
& \quad \leqslant \varepsilon\left(d \alpha_{1}\right)^{1 / 2}\|g\|_{L_{\infty}}\|\Lambda\|_{L_{\infty}}\left\|b(\mathbf{D}) \mathbf{D}\left(B_{D}^{0}\right)^{-5 / 2} \sin \left(t\left(B_{D}^{0}\right)^{1 / 2}\right)\right\|_{L_{2}(\mathcal{O}) \rightarrow L_{2}(\mathcal{O})} \\
& \quad+\varepsilon\left(d \alpha_{1}\right)^{1 / 2}\|g\|_{L_{\infty}}\|\widetilde{\Lambda}\|_{L_{p}(\Omega)} C_{\Omega}(p)\left\|P_{\mathcal{O}} \mathbf{D}\left(B_{D}^{0}\right)^{-5 / 2} \sin \left(t\left(B_{D}^{0}\right)^{1 / 2}\right)\right\|_{L_{2}(\mathcal{O}) \rightarrow H^{1}\left(\mathbb{R}^{d}\right)} .
\end{aligned}
$$

Together with (1.3), (1.57), and (2.15), this implies

$$
\left\|\varepsilon \sum_{l=1}^{d} g^{\varepsilon} b_{l}\left(\Lambda^{\varepsilon} b(\mathbf{D})+\widetilde{\Lambda}^{\varepsilon}\right) D_{l}\left(B_{D}^{0}\right)^{-5 / 2} \sin \left(t\left(B_{D}^{0}\right)^{1 / 2}\right)\right\|_{L_{2}(\mathcal{O}) \rightarrow L_{2}(\mathcal{O})} \leqslant \varepsilon|t| \widehat{C}_{12}, \quad t \in \mathbb{R},
$$

where $\widehat{C}_{12}:=\left(d \alpha_{1}\right)^{1 / 2} \mathcal{C}_{1} \mathcal{C}_{3}\|g\|_{L_{\infty}}\left(\left(d \alpha_{1}\right)^{1 / 2}\|\Lambda\|_{L_{\infty}}+C_{\Omega}(p) C_{\mathcal{O}}^{(1)}\|\widetilde{\Lambda}\|_{L_{p}(\Omega)}\right)$.

From (1.22) and (2.17)-(2.19) we derive estimate (2.12) with the constant

$$
C_{12}:=\left(d \alpha_{1}\right)^{1 / 2}\|g\|_{L_{\infty}} C_{11}+\widehat{C}_{12}
$$

2.5. Removal of the smoothing operator from the corrector for $3 \leqslant d \leqslant 8$. If $d \leqslant 2$, then, according to Propositions 2.5 and 2.7, Theorem 2.9 is applicable. So, let $d \geqslant 3$. Now we are interested in the possibility to remove the smoothing operator from the corrector without any additional assumptions on the matrix-valued functions $\Lambda$ and $\widetilde{\Lambda}$.

If $3 \leqslant d \leqslant 8$ and the boundary $\partial \mathcal{O}$ is sufficiently smooth, it turns out that the smoothing operator $S_{\varepsilon}$ can be eliminated from the both terms of the corrector. To do this, we use the properties of the matrix-valued functions $\Lambda^{\varepsilon}$ and $\widetilde{\Lambda}^{\varepsilon}$ as multipliers. The following result was obtained in [MSu5, Lemmas 6.3 and 6.5, Corollaries 6.4 and 6.6].

Lemma 2.13. Let the matrix-valued function $\Lambda(\mathbf{x})$ be the $\Gamma$-periodic solution of problem (1.21). Assume that $d \geqslant 3$ and put $l=d / 2$.

$1^{\circ}$. For $0<\varepsilon \leqslant 1$, the operator $\left[\Lambda^{\varepsilon}\right]$ is a continuous mapping from $H^{l-1}\left(\mathcal{O} ; \mathbb{C}^{m}\right)$ to $L_{2}\left(\mathcal{O} ; \mathbb{C}^{n}\right)$ and

$$
\left\|\left[\Lambda^{\varepsilon}\right]\right\|_{H^{l-1}(\mathcal{O}) \rightarrow L_{2}(\mathcal{O})} \leqslant C^{(0)} .
$$

$2^{\circ}$. Let $0<\varepsilon \leqslant 1$. Then for the function $\mathbf{u} \in H^{l}\left(\mathbb{R}^{d} ; \mathbb{C}^{m}\right)$ we have the inclusion $\Lambda^{\varepsilon} \mathbf{u} \in$ $H^{1}\left(\mathbb{R}^{d} ; \mathbb{C}^{n}\right)$ and the estimate

$$
\left\|\Lambda^{\varepsilon} \mathbf{u}\right\|_{H^{1}\left(\mathbb{R}^{d}\right)} \leqslant C^{(1)} \varepsilon^{-1}\|\mathbf{u}\|_{L_{2}\left(\mathbb{R}^{d}\right)}+C^{(2)}\|\mathbf{u}\|_{H^{l}\left(\mathbb{R}^{d}\right)} .
$$

The constants $C^{(0)}, C^{(1)}$, and $C^{(2)}$ depend on $m, d, \alpha_{0}, \alpha_{1},\|g\|_{L_{\infty}},\left\|g^{-1}\right\|_{L_{\infty}}$, and the parameters of the lattice $\Gamma$.

Lemma 2.14. Let the matrix-valued function $\widetilde{\Lambda}(\mathbf{x})$ be the $\Gamma$-periodic solution of problem (1.31). Assume that $d \geqslant 3$ and put $l=d / 2$. 
$1^{\circ}$. For $0<\varepsilon \leqslant 1$, the operator $\left[\widetilde{\Lambda}^{\varepsilon}\right]$ is a continuous mapping from $H^{l-1}\left(\mathcal{O} ; \mathbb{C}^{n}\right)$ to $L_{2}\left(\mathcal{O} ; \mathbb{C}^{n}\right)$ and

$$
\left\|\left[\widetilde{\Lambda}^{\varepsilon}\right]\right\|_{H^{l-1}(\mathcal{O}) \rightarrow L_{2}(\mathcal{O})} \leqslant \widetilde{C}^{(0)} .
$$

$2^{\circ}$. Let $0<\varepsilon \leqslant 1$. Then for $\mathbf{u} \in H^{l}\left(\mathbb{R}^{d} ; \mathbb{C}^{n}\right)$ we have the inclusion $\widetilde{\Lambda}^{\varepsilon} \mathbf{u} \in H^{1}\left(\mathbb{R}^{d} ; \mathbb{C}^{n}\right)$ and the estimate

$$
\left\|\widetilde{\Lambda}^{\varepsilon} \mathbf{u}\right\|_{H^{1}\left(\mathbb{R}^{d}\right)} \leqslant \widetilde{C}^{(1)} \varepsilon^{-1}\|\mathbf{u}\|_{H^{1}\left(\mathbb{R}^{d}\right)}+\widetilde{C}^{(2)}\|\mathbf{u}\|_{H^{l}\left(\mathbb{R}^{d}\right)} .
$$

The constants $\widetilde{C}^{(0)}, \widetilde{C}^{(1)}$, and $\widetilde{C}^{(2)}$ depend only on the problem data (1.7).

According to theorems about regularity of solutions of strongly elliptic systems (see, e. g., [McL, Theorem 4.18]), the following assertion holds true.

Lemma 2.15. Let $3 \leqslant d \leqslant 8$. Assume that $\partial \mathcal{O} \in C^{d / 2,1}$ if $d$ is even and $\partial \mathcal{O} \in C^{(d+1) / 2,1}$ if $d$ is odd. Then the operator $\left(B_{D}^{0}\right)^{-5 / 2}$ is a continuous mapping from $L_{2}\left(\mathcal{O} ; \mathbb{C}^{n}\right)$ to $H^{d / 2+1}\left(\mathcal{O} ; \mathbb{C}^{n}\right)$ and

$$
\left\|\left(B_{D}^{0}\right)^{-5 / 2}\right\|_{L_{2}(\mathcal{O}) \rightarrow H^{l+1}(\mathcal{O})} \leqslant \mathscr{C}_{l}, \quad l=d / 2 .
$$

Note that for any $d \geqslant 1$ and $\partial \mathcal{O} \in C^{4,1}$ the operator $\left(B_{D}^{0}\right)^{-5 / 2}: L_{2}\left(\mathcal{O} ; \mathbb{C}^{n}\right) \rightarrow H^{5}\left(\mathcal{O} ; \mathbb{C}^{n}\right)$ is continuous and

$$
\left\|\left(B_{D}^{0}\right)^{-5 / 2}\right\|_{L_{2}(\mathcal{O}) \rightarrow H^{5}(\mathcal{O})} \leqslant \mathcal{C}_{5} .
$$

Theorem 2.16. Suppose that the assumptions of Theorem 2.2 are satisfied. Let $3 \leqslant d \leqslant 8$ and let $\partial \mathcal{O}$ be subject to conditions of Lemma 2.15. Let $G_{D}^{0}(\varepsilon ; t)$ be the operator (2.10). Then for $t \in \mathbb{R}$ and $0<\varepsilon \leqslant \varepsilon_{1}$ we have

$$
\begin{aligned}
& \|\left(B_{D, \varepsilon}^{-1 / 2} \sin \left(t B_{D, \varepsilon}^{1 / 2}\right)-\left(I+\varepsilon \Lambda^{\varepsilon} b(\mathbf{D})+\varepsilon \widetilde{\Lambda}^{\varepsilon}\right)\left(B_{D}^{0}\right)^{-1 / 2} \sin \left(t\left(B_{D}^{0}\right)^{1 / 2}\right)\right) \\
& \quad \times\left(B_{D}^{0}\right)^{-2} \|_{L_{2}(\mathcal{O}) \rightarrow H^{1}(\mathcal{O})} \leqslant C_{13} \varepsilon^{1 / 2}\left(1+t^{6}\right), \\
& \left\|\left(g^{\varepsilon} b(\mathbf{D}) B_{D, \varepsilon}^{-1 / 2} \sin \left(t B_{D, \varepsilon}^{1 / 2}\right)-G_{D}^{0}(\varepsilon ; t)\right)\left(B_{D}^{0}\right)^{-2}\right\|_{L_{2}(\mathcal{O}) \rightarrow L_{2}(\mathcal{O})} \leqslant C_{14} \varepsilon^{1 / 2}\left(1+t^{6}\right) .
\end{aligned}
$$

The constants $C_{13}$ and $C_{14}$ depend only on the problem data (1.7).

Proof. By Proposition 1.1, Lemma 2.13 $\left(2^{\circ}\right)$, and (1.2),

$$
\begin{aligned}
\varepsilon \| & {\left[\Lambda^{\varepsilon}\right]\left(S_{\varepsilon}-I\right) b(\mathbf{D}) P_{\mathcal{O}}\left(B_{D}^{0}\right)^{-1 / 2} \sin \left(t\left(B_{D}^{0}\right)^{1 / 2}\right)\left(B_{D}^{0}\right)^{-2} \|_{L_{2}(\mathcal{O}) \rightarrow H^{1}(\mathcal{O})} } \\
& \leqslant C^{(1)}\left\|\left(S_{\varepsilon}-I\right) b(\mathbf{D}) P_{\mathcal{O}}\left(B_{D}^{0}\right)^{-5 / 2}\right\|_{L_{2}(\mathcal{O}) \rightarrow L_{2}\left(\mathbb{R}^{d}\right)} \\
& +\varepsilon C^{(2)}\left\|\left(S_{\varepsilon}-I\right) b(\mathbf{D}) P_{\mathcal{O}}\left(B_{D}^{0}\right)^{-5 / 2}\right\|_{L_{2}(\mathcal{O}) \rightarrow H^{l}\left(\mathbb{R}^{d}\right)} \\
& \leqslant r_{1} \alpha_{1}^{1 / 2} C^{(1)} \varepsilon\left\|\mathbf{D}^{2} P_{\mathcal{O}}\left(B_{D}^{0}\right)^{-5 / 2}\right\|_{L_{2}(\mathcal{O}) \rightarrow L_{2}\left(\mathbb{R}^{d}\right)} \\
& +2 \alpha_{1}^{1 / 2} C^{(2)} \varepsilon\left\|\mathbf{D} P_{\mathcal{O}}\left(B_{D}^{0}\right)^{-5 / 2}\right\|_{L_{2}(\mathcal{O}) \rightarrow H^{l}\left(\mathbb{R}^{d}\right)}
\end{aligned}
$$

Together with (1.57) and (2.20), this implies

$$
\begin{aligned}
& \varepsilon\left\|\left[\Lambda^{\varepsilon}\right]\left(S_{\varepsilon}-I\right) b(\mathbf{D}) P_{\mathcal{O}}\left(B_{D}^{0}\right)^{-1 / 2} \sin \left(t\left(B_{D}^{0}\right)^{1 / 2}\right)\left(B_{D}^{0}\right)^{-2}\right\|_{L_{2}(\mathcal{O}) \rightarrow H^{1}(\mathcal{O})} \\
& \leqslant \varepsilon \alpha_{1}^{1 / 2}\left(r_{1} C^{(1)} C_{\mathcal{O}}^{(2)}\left\|\left(B_{D}^{0}\right)^{-5 / 2}\right\|_{L_{2}(\mathcal{O}) \rightarrow H^{2}(\mathcal{O})}+2 C^{(2)} C_{\mathcal{O}}^{(l+1)}\left\|\left(B_{D}^{0}\right)^{-5 / 2}\right\|_{L_{2}(\mathcal{O}) \rightarrow H^{l+1}(\mathcal{O})}\right) \\
& \leqslant \varepsilon \alpha_{1}^{1 / 2}\left(r_{1} C^{(1)} C_{\mathcal{O}}^{(2)}+2 C^{(2)} C_{\mathcal{O}}^{(l+1)}\right) \mathscr{C}_{l}, \quad 3 \leqslant d=2 l \leqslant 8 .
\end{aligned}
$$

Similarly, using Lemma $2.14\left(2^{\circ}\right)$, we obtain

$$
\begin{aligned}
& \varepsilon\left\|\left[\widetilde{\Lambda}^{\varepsilon}\right]\left(S_{\varepsilon}-I\right) P_{\mathcal{O}}\left(B_{D}^{0}\right)^{-1 / 2} \sin \left(t\left(B_{D}^{0}\right)^{1 / 2}\right)\left(B_{D}^{0}\right)^{-2}\right\|_{L_{2}(\mathcal{O}) \rightarrow H^{1}(\mathcal{O})} \\
& \leqslant \varepsilon\left(r_{1} \widetilde{C}^{(1)} C_{\mathcal{O}}^{(2)}+2 \widetilde{C}^{(2)} C_{\mathcal{O}}^{(l)}\right) \mathscr{C}_{l}, \quad 3 \leqslant d=2 l \leqslant 8 .
\end{aligned}
$$


Combining (2.7), (2.24), and (2.25), we arrive at estimate (2.22) with the constant $C_{13}:=C_{8}+\alpha_{1}^{1 / 2}\left(r_{1} C^{(1)} C_{\mathcal{O}}^{(2)}+2 C^{(2)} C_{\mathcal{O}}^{(l+1)}\right) \mathscr{C}_{l}+\left(r_{1} \widetilde{C}^{(1)} C_{\mathcal{O}}^{(2)}+2 \widetilde{C}^{(2)} C_{\mathcal{O}}^{(l)}\right) \mathscr{C}_{l}$.

Now we proceed to the proof of inequality (2.23). By (1.3) and (2.22),

$$
\begin{aligned}
& \|\left(g^{\varepsilon} b(\mathbf{D}) B_{D, \varepsilon}^{-1 / 2} \sin \left(t B_{D, \varepsilon}^{1 / 2}\right)-g^{\varepsilon} b(\mathbf{D})\left(I+\varepsilon \Lambda^{\varepsilon} b(\mathbf{D})+\varepsilon \widetilde{\Lambda}^{\varepsilon}\right)\left(B_{D}^{0}\right)^{-1 / 2} \sin \left(t\left(B_{D}^{0}\right)^{1 / 2}\right)\right) \\
& \quad \times\left(B_{D}^{0}\right)^{-2}\left\|_{L_{2}(\mathcal{O}) \rightarrow L_{2}(\mathcal{O})} \leqslant\left(d \alpha_{1}\right)^{1 / 2}\right\| g \|_{L_{\infty}} C_{13} \varepsilon^{1 / 2}\left(1+t^{6}\right), \quad t \in \mathbb{R}, \quad 0<\varepsilon \leqslant \varepsilon_{1} .
\end{aligned}
$$

Identity (2.18) holds true. To estimate the fourth term in the right-hand side of (2.18), we apply Lemmas $2.13\left(1^{\circ}\right)$ and $2.14\left(1^{\circ}\right)$ and inequalities (1.3) and (2.20):

$$
\begin{aligned}
& \left\|\varepsilon \sum_{l=1}^{d} g^{\varepsilon} b_{l}\left(\Lambda^{\varepsilon} b(\mathbf{D})+\widetilde{\Lambda}^{\varepsilon}\right) D_{l}\left(B_{D}^{0}\right)^{-5 / 2} \sin \left(t\left(B_{D}^{0}\right)^{1 / 2}\right)\right\|_{L_{2}(\mathcal{O}) \rightarrow L_{2}(\mathcal{O})} \\
& \leqslant \varepsilon\|g\|_{L_{\infty}}\left(d \alpha_{1}\right)^{1 / 2}\left\|\left(\Lambda^{\varepsilon} b(\mathbf{D})+\widetilde{\Lambda}^{\varepsilon}\right) \mathbf{D}\left(B_{D}^{0}\right)^{-5 / 2}\right\|_{L_{2}(\mathcal{O}) \rightarrow L_{2}(\mathcal{O})} \\
& \leqslant \varepsilon\|g\|_{L_{\infty}}\left(d \alpha_{1}\right)^{1 / 2} \\
& \times\left(C^{(0)}\left(d \alpha_{1}\right)^{1 / 2}\left\|\mathbf{D}^{2}\left(B_{D}^{0}\right)^{-5 / 2}\right\|_{L_{2}(\mathcal{O}) \rightarrow H^{l-1}(\mathcal{O})}+\widetilde{C}^{(0)}\left\|\mathbf{D}\left(B_{D}^{0}\right)^{-5 / 2}\right\|_{L_{2}(\mathcal{O}) \rightarrow H^{l-1}(\mathcal{O})}\right) \\
& \leqslant \varepsilon \widehat{C}_{14}, \quad d \leqslant 8, \quad \widehat{C}_{14}:=\|g\|_{L_{\infty}}\left(d \alpha_{1}\right)^{1 / 2}\left(C^{(0)}\left(d \alpha_{1}\right)^{1 / 2}+\widetilde{C}^{(0)}\right) \mathscr{C}_{l} .
\end{aligned}
$$

Relations (2.26) and (2.27) imply the required estimate (2.23) with the constant

$$
C_{14}:=\left(d \alpha_{1}\right)^{1 / 2}\|g\|_{L_{\infty}} C_{13}+\widehat{C}_{14}
$$

Remark 2.17. If $\partial \mathcal{O} \in C^{4,1}$ and $d=9,10$, it is possible to remove the smoothing operator $S_{\varepsilon}$ only from the term of the corrector containing $\widetilde{\Lambda}^{\varepsilon}$. To do this, we use estimate (2.21) instead of Lemma 2.15.

2.6. Homogenization for the solution of the first initial-boundary value problem. Now we apply the results of Subsec. 2.2 and 2.4 to homogenization for the solution of the first initial-boundary value problem (2.1). Note that, if $\boldsymbol{\Phi} \in \operatorname{Dom}\left(B_{D}^{0}\right)^{2}$, then the function $\boldsymbol{\Phi}$ can be represented as $\boldsymbol{\Phi}=\left(B_{D}^{0}\right)^{-2} \check{\boldsymbol{\Phi}}$, where $\check{\boldsymbol{\Phi}} \in L_{2}\left(\mathcal{O} ; \mathbb{C}^{n}\right)$. By the theorems about regularity of solutions of the strongly elliptic systems (see [McL, Chapter 4]), if $\partial \mathcal{O} \in C^{3,1}$, then $\operatorname{Dom}\left(B_{D}^{0}\right)^{2} \subset H^{4}\left(\mathcal{O} ; \mathbb{C}^{n}\right)$. So, in this case, by Lemma 1.7 ,

$$
\|\check{\mathbf{\Phi}}\|_{L_{2}(\mathcal{O})}=\left\|\left(B_{D}^{0}\right)^{2} \boldsymbol{\Phi}\right\|_{L_{2}(\mathcal{O})} \leqslant \mathfrak{C}\|\boldsymbol{\Phi}\|_{H^{4}(\mathcal{O})} .
$$

Applying these considerations to the functions $\boldsymbol{\varphi}, \boldsymbol{\psi}$, and $\mathbf{F}(\cdot, t)$, using identities (2.2), (2.4), and Theorem [2.1, we obtain the following result.

Theorem 2.18. Let $\mathcal{O} \subset \mathbb{R}^{d}$ be a bounded domain of class $C^{3,1}$. Suppose that the assumptions of Subsec. 1.3 1.6 are satisfied. Let $\mathbf{u}_{\varepsilon}$ be the solution of problem (2.1) and let $\mathbf{u}_{0}$ be the solution of the effective problem (2.3), where $\boldsymbol{\varphi}, \boldsymbol{\psi} \in \operatorname{Dom}\left(B_{D}^{0}\right)^{2}$, and $\mathbf{F} \in$ $L_{1, \text { loc }}\left(\mathbb{R} ; \operatorname{Dom}\left(B_{D}^{0}\right)^{2}\right)$. Then for $t \in \mathbb{R}$ and $0<\varepsilon \leqslant \varepsilon_{1}$ we have

$$
\begin{aligned}
\left\|\mathbf{u}_{\varepsilon}(\cdot, t)-\mathbf{u}_{0}(\cdot, t)\right\|_{L_{2}(\mathcal{O})} & \leqslant \mathfrak{C} C_{7} \varepsilon\left(1+|t|^{5}\right) \\
& \times\left(\|\boldsymbol{\varphi}\|_{H^{4}(\mathcal{O})}+|t|\|\boldsymbol{\psi}\|_{H^{4}(\mathcal{O})}+|t|\|\mathbf{F}\|_{L_{1}\left((0, t) ; H^{4}(\mathcal{O})\right)}\right) .
\end{aligned}
$$

The constants $\mathfrak{C}$ and $C_{7}$ depend only on the problem data (1.7).

Using Theorems 2.1 and 2.2, we obtain approximation in the energy norm for the solution $\mathbf{u}_{\varepsilon}$ of problem (2.1) with $\varphi=0$. 
Theorem 2.19. Under the assumptions of Theorem 2.18 , let $\varphi=0$. Then for $t \in \mathbb{R}$ and $0<\varepsilon \leqslant \varepsilon_{1}$ we have

$$
\left\|\frac{\partial \mathbf{u}_{\varepsilon}}{\partial t}(\cdot, t)-\frac{\partial \mathbf{u}_{0}}{\partial t}(\cdot, t)\right\|_{L_{2}(\mathcal{O})} \leqslant \mathfrak{C} C_{7} \varepsilon\left(1+|t|^{5}\right)\left(\|\boldsymbol{\psi}\|_{H^{4}(\mathcal{O})}+\|\mathbf{F}\|_{L_{1}\left((0, t) ; H^{4}(\mathcal{O})\right)}\right) .
$$

Let $\Lambda(\mathbf{x})$ and $\widetilde{\Lambda}(\mathbf{x})$ be the $\Gamma$-periodic solutions of problems (1.21) and (1.31), respectively. Let $P_{\mathcal{O}}$ be the linear continuous extension operator (1.56) and let $S_{\varepsilon}$ be the Steklov smoothing operator (1.1). Put $\widetilde{\mathbf{u}}_{0}(\cdot, t):=P_{\mathcal{O}} \mathbf{u}_{0}(\cdot, t)$. By $\mathbf{v}_{\varepsilon}(\cdot, t)$ we denote the first order approximation for the solution $\mathbf{u}_{\varepsilon}(\cdot, t)$ :

$$
\widetilde{\mathbf{v}}_{\varepsilon}(\cdot, t):=\widetilde{\mathbf{u}}_{0}(\cdot, t)+\varepsilon \Lambda^{\varepsilon} S_{\varepsilon} b(\mathbf{D}) \widetilde{\mathbf{u}}_{0}(\cdot, t)+\varepsilon \widetilde{\Lambda}^{\varepsilon} S_{\varepsilon} \widetilde{\mathbf{u}}_{0}(\cdot, t), \quad \mathbf{v}_{\varepsilon}(\cdot, t):=\left.\widetilde{\mathbf{v}}_{\varepsilon}(\cdot, t)\right|_{\mathcal{O}} .
$$

Then for $t \in \mathbb{R}$ and $0<\varepsilon \leqslant \varepsilon_{1}$ we have

$$
\left\|\mathbf{u}_{\varepsilon}(\cdot, t)-\mathbf{v}_{\varepsilon}(\cdot, t)\right\|_{H^{1}(\mathcal{O})} \leqslant \mathfrak{C} C_{8} \varepsilon^{1 / 2}\left(1+t^{6}\right)\left(\|\boldsymbol{\psi}\|_{H^{4}(\mathcal{O})}+\|\mathbf{F}\|_{L_{1}\left((0, t) ; H^{4}(\mathcal{O})\right)}\right) .
$$

Let $\widetilde{g}(\mathbf{x})$ be the matrix-valued function (1.22). Let $\mathbf{p}_{\varepsilon}(\cdot, t):=g^{\varepsilon} b(\mathbf{D}) \mathbf{u}_{\varepsilon}(\cdot, t)$. Then for $t \in \mathbb{R}$ and $0<\varepsilon \leqslant \varepsilon_{1}$ we have

$$
\begin{aligned}
& \left\|\mathbf{p}_{\varepsilon}(\cdot, t)-\widetilde{g}^{\varepsilon} S_{\varepsilon} b(\mathbf{D}) \widetilde{\mathbf{u}}_{0}(\cdot, t)-g^{\varepsilon}(b(\mathbf{D}) \widetilde{\Lambda})^{\varepsilon} S_{\varepsilon} \widetilde{\mathbf{u}}_{0}(\cdot, t)\right\|_{L_{2}(\mathcal{O})} \\
& \quad \leqslant \mathfrak{C} C_{9} \varepsilon^{1 / 2}\left(1+t^{6}\right)\left(\|\boldsymbol{\psi}\|_{H^{4}(\mathcal{O})}+\|\mathbf{F}\|_{L_{1}\left((0, t) ; H^{4}(\mathcal{O})\right)}\right) .
\end{aligned}
$$

The constants $\mathfrak{C}, C_{8}$, and $C_{9}$ depend only on the problem data (1.7).

Proof. Estimates (2.30) and (2.31) follow from Lemma 1.7, Theorem 2.2, and relations (2.2), (2.4).

Let us discuss the proof of inequality (2.29). We set $\boldsymbol{\varphi}=0$ in (2.2) and differentiate the obtained identity with respect to $t$. Then

$$
\frac{\partial \mathbf{u}_{\varepsilon}}{\partial t}(\cdot, t)=\cos \left(t B_{D, \varepsilon}^{1 / 2}\right) \boldsymbol{\psi}+\int_{0}^{t} \cos \left((t-\widetilde{t}) B_{D, \varepsilon}^{1 / 2}\right) \mathbf{F}(\cdot, \widetilde{t}) d \widetilde{t}
$$

The similar identity holds for the solution of the effective problem. Together with Lemma 1.7 and Theorem 2.1, this implies estimate (2.29).

From Theorem 2.16 we derive the following result.

Theorem 2.20. Under the assumptions of Theorem 2.19 , let $d \leqslant 8$. If $d=7,8$, we additionally assume that $\partial \mathcal{O} \in C^{4,1}$. Denote $\check{\mathbf{v}}_{\varepsilon}(\cdot, t):=\mathbf{u}_{0}(\cdot, t)+\varepsilon\left(\Lambda^{\varepsilon} b(\mathbf{D})+\widetilde{\Lambda}^{\varepsilon}\right) \mathbf{u}_{0}(\cdot, t)$. Then for $t \in \mathbb{R}$ and $0<\varepsilon \leqslant \varepsilon_{1}$ we have

$$
\begin{aligned}
& \left\|\mathbf{u}_{\varepsilon}(\cdot, t)-\check{\mathbf{v}}_{\varepsilon}(\cdot, t)\right\|_{H^{1}(\mathcal{O})} \leqslant \mathfrak{C} C_{13} \varepsilon^{1 / 2}\left(1+t^{6}\right)\left(\|\boldsymbol{\psi}\|_{H^{4}(\mathcal{O})}+\|\mathbf{F}\|_{L_{1}\left((0, t) ; H^{4}(\mathcal{O})\right)}\right), \\
& \left\|\mathbf{p}_{\varepsilon}(\cdot, t)-\widetilde{g}^{\varepsilon} b(\mathbf{D}) \mathbf{u}_{0}(\cdot, t)-g^{\varepsilon}(b(\mathbf{D}) \widetilde{\Lambda})^{\varepsilon} \mathbf{u}_{0}(\cdot, t)\right\|_{L_{2}(\mathcal{O})} \\
& \quad \leqslant \mathfrak{C} C_{14} \varepsilon^{1 / 2}\left(1+t^{6}\right)\left(\|\boldsymbol{\psi}\|_{H^{4}(\mathcal{O})}+\|\mathbf{F}\|_{L_{1}\left((0, t) ; H^{4}(\mathcal{O})\right)}\right)
\end{aligned}
$$

The constants $\mathfrak{C}, C_{13}$, and $C_{14}$ depend only on the problem data (1.7).

Remark 2.21. If $\partial \mathcal{O} \in C^{1,1}$, the results of Theorems 2.18, 2.19, and 2.20 remain true with the norms $\left\|\left(B_{D}^{0}\right)^{2} \boldsymbol{\varphi}\right\|_{L_{2}(\mathcal{O})},\left\|\left(B_{D}^{0}\right)^{2} \boldsymbol{\psi}\right\|_{L_{2}(\mathcal{O})}$, and $\left\|\left(B_{D}^{0}\right)^{2} \mathbf{F}\right\|_{L_{1}\left((0, t) ; L_{2}(\mathcal{O})\right)}$ instead of $\|\boldsymbol{\varphi}\|_{H^{4}(\mathcal{O})}$, $\|\boldsymbol{\psi}\|_{H^{4}(\mathcal{O})}$, and $\|\mathbf{F}\|_{L_{1}\left((0, t) ; H^{4}(\mathcal{O})\right)}$, respectively, in the error estimates.

2.7. The special case. Assume that $g^{0}=g$, i. e., relations (1.30) are satisfied. Then, by Proposition 2.5 $\left(3^{\circ}\right)$, Condition 2.4 holds. Herewith, according to [BSu2, Remark 3.5], the matrix-valued function (1.22) is constant and coincides with $g^{0}$, i. e., $\widetilde{g}(\mathbf{x})=g^{0}=g$. Thus, $\widetilde{g}^{\varepsilon} b(\mathbf{D}) \mathbf{u}_{0}(\cdot, t)=g^{0} b(\mathbf{D}) \mathbf{u}_{0}(\cdot, t)$.

In addition, suppose that

$$
\sum_{j=1}^{d} D_{j} a_{j}(\mathbf{x})^{*}=0 .
$$


Then the $\Gamma$-periodic solution of problem (1.31) is also equal to zero: $\widetilde{\Lambda}(\mathbf{x})=0$. So, Theorem 2.9 implies the following result.

Proposition 2.22. Under the assumptions of Theorem 2.19, suppose that relations (1.30) and (2.32) hold. Then for $t \in \mathbb{R}$ and $0<\varepsilon \leqslant \varepsilon_{1}$ we have

$$
\left\|\mathbf{p}_{\varepsilon}(\cdot, t)-g^{0} b(\mathbf{D}) \mathbf{u}_{0}(\cdot, t)\right\|_{L_{2}(\mathcal{O})} \leqslant \mathfrak{C} C_{12} \varepsilon^{1 / 2}\left(1+t^{6}\right)\left(\|\boldsymbol{\psi}\|_{H^{4}(\mathcal{O})}+\|\mathbf{F}\|_{L_{1}\left((0, t) ; H^{4}(\mathcal{O})\right)}\right) .
$$

2.8. The case where the corrector is equal to zero. Assume that $g^{0}=\bar{g}$, i. e., relations (1.29) are satisfied. Assume that condition (2.32) holds. Then the $\Gamma$-periodic solutions of problems (1.21) and (1.31) are equal to zero: $\Lambda(\mathbf{x})=0$ and $\widetilde{\Lambda}(\mathbf{x})=0$. By Theorems 2.2 and 2.3. for $t \in \mathbb{R}$ and $0<\varepsilon \leqslant \varepsilon_{1}$ we have

$$
\begin{aligned}
& \left\|\left(B_{D, \varepsilon}^{-1 / 2} \sin \left(t B_{D, \varepsilon}^{1 / 2}\right)-\left(B_{D}^{0}\right)^{-1 / 2} \sin \left(t\left(B_{D}^{0}\right)^{1 / 2}\right)\right)\left(B_{D}^{0}\right)^{-2}\right\|_{L_{2}(\mathcal{O}) \rightarrow H^{1}(\mathcal{O})} \leqslant C_{8} \varepsilon^{1 / 2}\left(1+t^{6}\right) \\
& \left\|\left(\cos \left(t B_{D, \varepsilon}^{1 / 2}\right) B_{D, \varepsilon}^{-1}-\cos \left(t\left(B_{D}^{0}\right)^{1 / 2}\right)\left(B_{D}^{0}\right)^{-1}\right)\left(B_{D}^{0}\right)^{-1}\right\|_{L_{2}(\mathcal{O}) \rightarrow H^{1}(\mathcal{O})} \leqslant C_{10} \varepsilon^{1 / 2}\left(1+|t|^{5}\right) .
\end{aligned}
$$

In the case under consideration, Theorem $1.10\left(2^{\circ}\right)$ implies that

$$
\left\|B_{D, \varepsilon}^{-1}-\left(B_{D}^{0}\right)^{-1}\right\|_{L_{2}(\mathcal{O}) \rightarrow H^{1}(\mathcal{O})} \leqslant C_{4} \max \left\{2 ; c_{\mathrm{b}}^{-1}+c_{\mathrm{b}}^{-2}\right\} \varepsilon^{1 / 2}, \quad 0<\varepsilon \leqslant \varepsilon_{1} .
$$

Applying (1.18) and (1.16) consistently, we obtain

$$
\begin{aligned}
& \left\|\cos \left(t B_{D, \varepsilon}^{1 / 2}\right)\left(B_{D, \varepsilon}^{-1}-\left(B_{D}^{0}\right)^{-1}\right)\left(B_{D}^{0}\right)^{-1}\right\|_{L_{2}(\mathcal{O}) \rightarrow H^{1}(\mathcal{O})} \\
& \quad \leqslant c_{3}\left\|B_{D, \varepsilon}^{1 / 2} \cos \left(t B_{D, \varepsilon}^{1 / 2}\right)\left(B_{D, \varepsilon}^{-1}-\left(B_{D}^{0}\right)^{-1}\right)\left(B_{D}^{0}\right)^{-1}\right\|_{L_{2}(\mathcal{O}) \rightarrow L_{2}(\mathcal{O})} \\
& \leqslant c_{3} C_{*}^{1 / 2}\left\|B_{D, \varepsilon}^{-1}-\left(B_{D}^{0}\right)^{-1}\right\|_{L_{2}(\mathcal{O}) \rightarrow H^{1}(\mathcal{O})}\left\|\left(B_{D}^{0}\right)^{-1}\right\|_{L_{2}(\mathcal{O}) \rightarrow L_{2}(\mathcal{O}) .}
\end{aligned}
$$

Combining (1.40) and (2.34) $-(2.36)$, for $t \in \mathbb{R}$ and $0<\varepsilon \leqslant \varepsilon_{1}$ we have

$$
\left\|\left(\cos \left(t B_{D, \varepsilon}^{1 / 2}\right)-\cos \left(t\left(B_{D}^{0}\right)^{1 / 2}\right)\right)\left(B_{D}^{0}\right)^{-2}\right\|_{L_{2}(\mathcal{O}) \rightarrow H^{1}(\mathcal{O})} \leqslant C_{15} \varepsilon^{1 / 2}\left(1+|t|^{5}\right) .
$$

Here $C_{15}:=C_{10}+c_{3} C_{*}^{1 / 2} C_{4} \max \left\{2 ; c_{b}^{-1}+c_{b}^{-2}\right\} \mathcal{C}_{1}$.

Bringing together (2.28), (2.33), and (2.37), we arrive at approximation in the Sobolev class $H^{1}\left(\mathcal{O} ; \mathbb{C}^{n}\right)$ for the solution (2.2) of the problem (2.1).

Proposition 2.23. Let $\mathbf{u}_{\varepsilon}$ and $\mathbf{u}_{0}$ be solutions of problems (2.1) and (2.3), respectively, for $\boldsymbol{\varphi}, \boldsymbol{\psi} \in \operatorname{Dom}\left(B_{D}^{0}\right)^{2}$, and $\mathbf{F} \in L_{1, \text { loc }}\left(\mathbb{R} ; \operatorname{Dom}\left(B_{D}^{0}\right)^{2}\right)$. Assume that relations (1.29) and (2.32) hold true. Then for $0<\varepsilon \leqslant \varepsilon_{1}$ and $t \in \mathbb{R}$ we have

$$
\begin{aligned}
\left\|\mathbf{u}_{\varepsilon}(\cdot, t)-\mathbf{u}_{0}(\cdot, t)\right\|_{H^{1}(\mathcal{O})} & \leqslant \mathfrak{C} C_{15} \varepsilon^{1 / 2}\left(1+|t|^{5}\right)\|\boldsymbol{\varphi}\|_{H^{4}(\mathcal{O})} \\
& +\mathfrak{C} C_{8} \varepsilon^{1 / 2}\left(1+t^{6}\right)\left(\|\boldsymbol{\psi}\|_{H^{4}(\mathcal{O})}+\|\mathbf{F}\|_{L_{1}\left((0, t) ; H^{4}(\mathcal{O})\right)}\right) .
\end{aligned}
$$

\section{Proof of Theorems 2.1 and 2.2}

3.1. Proof of Theorem 2.1. To prove estimate (2.5), we use the inverse Laplace transform and Theorem 1.9. To guarantee the convergence of the corresponding integrals, we consider the function $\left(\cos \left(t a^{1 / 2}\right)-1+a t^{2} / 2\right) a^{-2}$ instead of the cosine. The reason is that the inverse Laplace transform of this function decreases faster than the inverse Laplace transform of the cosine (see, e. g., GraRy, Section 17.13]).

Proof of Theorem 2.1. For $t=0$, the result (2.5) is trivial:

$$
\left.\cos \left(t B_{D, \varepsilon}^{1 / 2}\right)\right|_{t=0}=\left.\cos \left(t\left(B_{D}^{0}\right)^{1 / 2}\right)\right|_{t=0}=I .
$$

Therefore, since the cosine is an even function, without loss of generality, we will further assume that $t>0$.

By (1.53) and (1.54),

$$
\left\|B_{D, \varepsilon}^{-1}-\left(B_{D}^{0}\right)^{-1}\right\|_{L_{2}(\mathcal{O}) \rightarrow L_{2}(\mathcal{O})} \leqslant C_{16} \varepsilon, \quad C_{16}:=\max \left\{1 ; c_{b}^{-2}\right\} C_{2} .
$$


So, by using the identity

$$
\begin{aligned}
B_{D, \varepsilon}^{-2}-\left(B_{D}^{0}\right)^{-2} & =\frac{1}{2}\left(B_{D, \varepsilon}^{-1}-\left(B_{D}^{0}\right)^{-1}\right)\left(B_{D, \varepsilon}^{-1}+\left(B_{D}^{0}\right)^{-1}\right) \\
& +\frac{1}{2}\left(B_{D, \varepsilon}^{-1}+\left(B_{D}^{0}\right)^{-1}\right)\left(B_{D, \varepsilon}^{-1}-\left(B_{D}^{0}\right)^{-1}\right)
\end{aligned}
$$

and estimates (1.19), (1.40), we obtain

$$
\left\|B_{D, \varepsilon}^{-2}-\left(B_{D}^{0}\right)^{-2}\right\|_{L_{2}(\mathcal{O}) \rightarrow L_{2}(\mathcal{O})} \leqslant 2 \mathcal{C}_{1} C_{16} \varepsilon .
$$

Thus,

$$
\begin{aligned}
& \left\|\left(\cos \left(t B_{D, \varepsilon}^{1 / 2}\right)-\cos \left(t\left(B_{D}^{0}\right)^{1 / 2}\right)\right)\left(B_{D}^{0}\right)^{-2}\right\|_{L_{2}(\mathcal{O}) \rightarrow L_{2}(\mathcal{O})} \\
& \quad \leqslant\left\|\cos \left(t B_{D, \varepsilon}^{1 / 2}\right)\left(B_{D, \varepsilon}^{-2}-\left(B_{D}^{0}\right)^{-2}\right)\right\|_{L_{2}(\mathcal{O}) \rightarrow L_{2}(\mathcal{O})} \\
& \quad+\left\|\cos \left(t B_{D, \varepsilon}^{1 / 2}\right) B_{D, \varepsilon}^{-2}-\cos \left(t\left(B_{D}^{0}\right)^{1 / 2}\right)\left(B_{D}^{0}\right)^{-2}\right\|_{L_{2}(\mathcal{O}) \rightarrow L_{2}(\mathcal{O})} \\
& \quad \leqslant 2 \mathcal{C}_{1} C_{16} \varepsilon+\left\|\cos \left(t B_{D, \varepsilon}^{1 / 2}\right) B_{D, \varepsilon}^{-2}-\cos \left(t\left(B_{D}^{0}\right)^{1 / 2}\right)\left(B_{D}^{0}\right)^{-2}\right\|_{L_{2}(\mathcal{O}) \rightarrow L_{2}(\mathcal{O})} .
\end{aligned}
$$

Let $a>0$ be a parameter. Then, by the residue theorem,

$$
\left(\frac{1}{2} a t^{2}-1+\cos (t \sqrt{a})\right) a^{-2}=\frac{1}{2 \pi i} \int_{\operatorname{Re} \lambda=c} \lambda^{-3}\left(a+\lambda^{2}\right)^{-1} e^{\lambda t} d \lambda, \quad c>0 .
$$

Assume that the constant $c$ in (3.4) is equal to $\sqrt{c_{b}} / t$. With the help of the spectral theorem, from (3.4) we derive

$$
\left(\frac{1}{2} B_{D, \varepsilon} t^{2}-I+\cos \left(t B_{D, \varepsilon}^{1 / 2}\right)\right) B_{D, \varepsilon}^{-2}=\frac{1}{2 \pi i} \int_{\operatorname{Re} \lambda=\sqrt{c_{b}} / t} \lambda^{-3}\left(B_{D, \varepsilon}+\lambda^{2} I\right)^{-1} e^{\lambda t} d \lambda .
$$

The similar identity holds for the effective operator. So,

$$
\begin{gathered}
\cos \left(t B_{D, \varepsilon}^{1 / 2}\right) B_{D, \varepsilon}^{-2}-\cos \left(t\left(B_{D}^{0}\right)^{1 / 2}\right)\left(B_{D}^{0}\right)^{-2}=-\frac{t^{2}}{2}\left(B_{D, \varepsilon}^{-1}-\left(B_{D}^{0}\right)^{-1}\right)+\left(B_{D, \varepsilon}^{-2}-\left(B_{D}^{0}\right)^{-2}\right) \\
+\frac{1}{2 \pi i} \int_{\operatorname{Re} \lambda=\sqrt{c_{b}} / t} \lambda^{-3}\left(\left(B_{D, \varepsilon}+\lambda^{2} I\right)^{-1}-\left(B_{D}^{0}+\lambda^{2} I\right)^{-1}\right) e^{\lambda t} d \lambda .
\end{gathered}
$$

Combining this with (3.1) and (3.2), we conclude that

$$
\begin{aligned}
& \left\|\cos \left(t B_{D, \varepsilon}^{1 / 2}\right) B_{D, \varepsilon}^{-2}-\cos \left(t\left(B_{D}^{0}\right)^{1 / 2}\right)\left(B_{D}^{0}\right)^{-2}\right\|_{L_{2}(\mathcal{O}) \rightarrow L_{2}(\mathcal{O})} \leqslant 2^{-1} C_{16} \varepsilon t^{2}+2 \mathcal{C}_{1} C_{16} \varepsilon \\
& +\left\|\frac{1}{2 \pi i} \int_{\operatorname{Re} \lambda=\sqrt{c_{b}} / t} \lambda^{-3}\left(\left(B_{D, \varepsilon}+\lambda^{2} I\right)^{-1}-\left(B_{D}^{0}+\lambda^{2} I\right)^{-1}\right) e^{\lambda t} d \lambda\right\|_{L_{2}(\mathcal{O}) \rightarrow L_{2}(\mathcal{O})}
\end{aligned}
$$

Now we proceed to estimation of the integral in the right-hand side of (3.7). By the change of variables $\lambda t=\mu$,

$$
\begin{aligned}
& \frac{1}{2 \pi i} \int_{\operatorname{Re} \lambda=\sqrt{c_{b}} / t} \lambda^{-3}\left(\left(B_{D, \varepsilon}+\lambda^{2} I\right)^{-1}-\left(B_{D}^{0}+\lambda^{2} I\right)^{-1}\right) e^{\lambda t} d \lambda \\
& =\frac{t^{2}}{2 \pi i} \int_{\operatorname{Re} \mu=\sqrt{c_{b}}} e^{\mu} \mu^{-3}\left(\left(B_{D, \varepsilon}+\frac{\mu^{2}}{t^{2}} I\right)^{-1}-\left(B_{D}^{0}+\frac{\mu^{2}}{t^{2}} I\right)^{-1}\right) d \mu=: \mathfrak{I}(\varepsilon ; t) .
\end{aligned}
$$

Substitute $\mu=\sqrt{c_{b}}+i \beta, \beta \in \mathbb{R}$. Denote $\zeta_{t}:=-\mu^{2} / t^{2}$. Let us understand how the set of values of this variable looks like. We have

$$
\mu^{2}=c_{b}-\beta^{2}+i 2 \beta \sqrt{c_{b}}=: x+i y .
$$

Then

$$
\left\{\begin{array}{l}
x=c_{b}-\beta^{2} \\
y=2 \beta \sqrt{c_{b}}
\end{array}\right.
$$


So,

$$
\left\{\begin{array}{l}
x=c_{b}-\left(4 c_{b}\right)^{-1} y^{2} \\
\beta=2^{-1} c_{b}^{-1 / 2} y
\end{array}\right.
$$

Thus, the values of $\zeta_{t}$ belong to the parabola $\Pi_{t}$ :

$$
\Pi_{t}:=\left\{\zeta_{t} \in \mathbb{C}: \operatorname{Re} \zeta_{t}=-\frac{c_{b}}{t^{2}}+\frac{t^{2}}{4 c_{b}}\left(\operatorname{Im} \zeta_{t}\right)^{2}\right\} .
$$

For $\zeta_{t} \in \Pi_{t}$ with $\operatorname{Re} \zeta_{t}<c_{b}+1$, we use approximation (1.54) for the resolvent $\left(B_{D, \varepsilon}-\zeta_{t} I\right)^{-1}$. Let us estimate $\varrho_{b}\left(\zeta_{t}\right)$ for $\zeta_{t} \in \Pi_{t}$ under consideration. We have

$$
\zeta_{t}=-\frac{\mu^{2}}{t^{2}}=\frac{\beta^{2}-c_{b}}{t^{2}}-i \frac{2 \beta \sqrt{c_{b}}}{t^{2}}, \quad \beta \in \mathbb{R} .
$$

So,

$$
\left|\zeta_{t}-c_{b}\right|^{2}=\left(\frac{\beta^{2}-c_{b}}{t^{2}}-c_{b}\right)^{2}+\frac{4 \beta^{2} c_{b}}{t^{4}} .
$$

After elementary transformations,

$$
\left|\zeta_{t}-c_{b}\right|^{2}=t^{-4}\left(\left(\beta^{2}-c_{b} t^{2}\right)^{2}+2 \beta^{2} c_{b}+c_{b}^{2}+2 c_{b}^{2} t^{2}\right) .
$$

Consequently, $\left|\zeta_{t}-c_{b}\right|^{-2} \leqslant\left(2 c_{b}^{2}\right)^{-1} t^{2}$. For $\zeta_{t} \in \Pi_{t}$ with $\operatorname{Re} \zeta_{t} \leqslant c_{b}$, we use the estimate

$$
\varrho_{b}\left(\zeta_{t}\right) \leqslant \max \left\{1 ;\left|\zeta_{t}-c_{b}\right|^{-2}\right\} \leqslant \max \left\{1 ;\left(2 c_{b}^{2}\right)^{-1} t^{2}\right\} \leqslant \mathfrak{c}_{1}\left(t^{2}+1\right) ; \quad \mathfrak{c}_{1}:=\max \left\{1 ;\left(2 c_{b}^{2}\right)^{-1}\right\} .
$$

Let $\psi_{t}=\arg \left(\zeta_{t}-c_{b}\right)$. For $\zeta_{t} \in \Pi_{t}$ with $c_{b}<\operatorname{Re} \zeta_{t} \leqslant c_{b}+1$, the value of $\varrho_{b}\left(\zeta_{t}\right)$ can be estimated as follows:

$$
\varrho_{b}\left(\zeta_{t}\right) \leqslant \max \left\{c\left(\psi_{t}\right)^{2}\left|\zeta_{t}-c_{b}\right|^{-2} ; c\left(\psi_{t}\right)^{2}\right\}=\max \left\{\left|\operatorname{Im}\left(\zeta_{t}-c_{b}\right)\right|^{-2} ; c\left(\psi_{t}\right)^{2}\right\} .
$$

We have

$$
\left|\operatorname{Im}\left(\zeta_{t}-c_{b}\right)\right|^{-2}=\left|\operatorname{Im} \zeta_{t}\right|^{-2} \leqslant\left|\operatorname{Im} \widehat{\zeta}_{t}\right|^{-2}, \quad \zeta_{t} \in \Pi_{t}, c_{b}<\operatorname{Re} \zeta_{t} \leqslant c_{b}+1 .
$$

Here $\widehat{\zeta}_{t}$ is the point at the contour $\Pi_{t}$ such that Re $\widehat{\zeta}_{t}=c_{b}$. (There are two such points, one can choose any.) Let $\widehat{\beta} \in \mathbb{R}$ be the corresponding value of the parameter $\beta$. Then $\operatorname{Re} \widehat{\zeta}_{t}=c_{b}=t^{-2}\left(\widehat{\beta}^{2}-c_{b}\right)$. So, $\widehat{\beta}^{2}=c_{b}\left(1+t^{2}\right)$. And, by (3.14) , for $\zeta_{t} \in \Pi_{t}$ under consideration we have

$$
\left|\operatorname{Im}\left(\zeta_{t}-c_{b}\right)\right|^{-2}=\left|\operatorname{Im} \zeta_{t}\right|^{-2} \leqslant \frac{t^{4}}{4 c_{b}} \widehat{\beta}^{-2}=\frac{t^{4}}{4 c_{b}^{2}\left(1+t^{2}\right)} \leqslant \frac{t^{2}}{4 c_{b}^{2}} .
$$

Now we want to estimate $c\left(\psi_{t}\right)$. Obviously, for $\zeta_{t} \in \Pi_{t}$ with $c_{b}<\operatorname{Re} \zeta_{t} \leqslant c_{b}+1$ we have $c\left(\psi_{t}\right) \leqslant c\left(\widetilde{\psi}_{t}\right)$, where $\widetilde{\psi}_{t}=\arg \widetilde{\zeta}_{t}, \widetilde{\zeta}_{t} \in \Pi_{t}, \operatorname{Re} \widetilde{\zeta}_{t}=c_{b}+1$. (There are two such points on the contour.) Assume that the point $\widetilde{\zeta}_{t} \in \Pi_{t}$ corresponds to the parameter $\widetilde{\beta}>0$. Then $c_{b}+1=\operatorname{Re} \widetilde{\zeta}_{t}=t^{-2}\left(\widetilde{\beta}^{2}-c_{b}\right)$. So,

$$
\widetilde{\beta}^{2}=c_{b}+t^{2}\left(c_{b}+1\right) .
$$

Thus,

$$
\left(\operatorname{Im} \widetilde{\zeta}_{t}\right)^{2}=\frac{4 \widetilde{\beta}^{2} c_{b}}{t^{4}}=\frac{4 c_{b}^{2}+4 c_{b} t^{2}\left(c_{b}+1\right)}{t^{4}}
$$

Next,

$$
c\left(\widetilde{\psi}_{t}\right)^{2}=\frac{\left|\widetilde{\zeta}_{t}-c_{b}\right|^{2}}{\left(\operatorname{Im} \widetilde{\zeta}_{t}\right)^{2}}=\frac{\left(\operatorname{Re}\left(\widetilde{\zeta}_{t}-c_{b}\right)\right)^{2}+\left(\operatorname{Im} \widetilde{\zeta}_{t}\right)^{2}}{\left(\operatorname{Im} \widetilde{\zeta}_{t}\right)^{2}}=\frac{t^{4}+4 c_{b}^{2}+4 c_{b} t^{2}\left(c_{b}+1\right)}{4 c_{b}^{2}+4 c_{b} t^{2}\left(c_{b}+1\right)}
$$


By the elementary inequality $1 \leqslant\left(c_{b}+1\right)^{2}$,

$$
c\left(\widetilde{\psi}_{t}\right)^{2} \leqslant \frac{t^{4}\left(c_{b}+1\right)^{2}+4 c_{b}^{2}+4 c_{b} t^{2}\left(c_{b}+1\right)}{4 c_{b}^{2}+4 c_{b} t^{2}\left(c_{b}+1\right)}=\frac{\left(t^{2}\left(c_{b}+1\right)+2 c_{b}\right)^{2}}{4 c_{b}^{2}+4 c_{b} t^{2}\left(c_{b}+1\right)} .
$$

By decreasing the denominator, we obtain

$$
c\left(\widetilde{\psi}_{t}\right)^{2} \leqslant \frac{\left(t^{2}\left(c_{b}+1\right)+2 c_{b}\right)^{2}}{2 c_{b}^{2}+c_{b} t^{2}\left(c_{b}+1\right)}=\frac{t^{2}\left(c_{b}+1\right)+2 c_{b}}{c_{b}}=2+\left(1+c_{b}^{-1}\right) t^{2} .
$$

Thus, by (3.13) and (3.15), for $\zeta_{t} \in \Pi_{t}$ with $c_{b}<\operatorname{Re} \zeta_{t} \leqslant c_{b}+1$ we have

(3.17) $\varrho_{b}\left(\zeta_{t}\right) \leqslant \max \left\{\left(2 c_{b}\right)^{-2} t^{2} ; 2+\left(1+c_{b}^{-1}\right) t^{2}\right\} \leqslant \mathfrak{c}_{2}\left(t^{2}+1\right) ; \quad \mathfrak{c}_{2}:=\max \left\{\left(2 c_{b}\right)^{-2} ; 2 ; 1+c_{b}^{-1}\right\}$.

Bringing together (3.12) and (3.17), we arrive at the estimate

$$
\varrho_{b}\left(\zeta_{t}\right) \leqslant \mathfrak{c}_{3}\left(1+t^{2}\right), \quad \zeta_{t} \in \Pi_{t}, \operatorname{Re} \zeta_{t} \leqslant c_{b}+1 ; \quad \mathfrak{c}_{3}:=\max \left\{\mathfrak{c}_{1} ; \mathfrak{c}_{2}\right\} .
$$

Let now $\zeta_{t} \in \Pi_{t}$ with $\operatorname{Re} \zeta_{t}>c_{b}+1$. For this part of the contour, we will use (1.52) to estimate the integrand in (3.8). Let $\phi_{t}=\arg \zeta_{t}$. By (3.11),

$$
t^{2}\left|\mu^{-3}\right|\left|\zeta_{t}\right|^{-1 / 2} c\left(\phi_{t}\right)^{2}=t^{-1}\left|\zeta_{t}\right|^{-2} c\left(\phi_{t}\right)^{2}=t^{-1}\left|\operatorname{Im} \zeta_{t}\right|^{-2}=\left(4 c_{b}\right)^{-1} t^{3} \beta^{-2} .
$$

Now, we can estimate the integral (3.8):

$$
\mathfrak{I}(\varepsilon ; t)=\frac{t^{2}}{2 \pi} \int_{-\infty}^{\infty} e^{\sqrt{c_{b}}} e^{i \beta}(\mu(\beta))^{-3}\left(\left(B_{D, \varepsilon}+\frac{\mu(\beta)^{2}}{t^{2}} I\right)^{-1}-\left(B_{D}^{0}+\frac{\mu(\beta)^{2}}{t^{2}} I\right)^{-1}\right) d \beta .
$$

Here $\mu(\beta)=c_{b}^{1 / 2}+i \beta$. Combining this with (1.52), (1.54), (3.18), and (3.19), we obtain

$$
\begin{aligned}
& \|\Im(\varepsilon ; t)\|_{L_{2}(\mathcal{O}) \rightarrow L_{2}(\mathcal{O})} \\
& \leqslant \frac{e^{\sqrt{c_{b}}} t^{2}}{2 \pi}\left(\varepsilon C_{2} \mathfrak{c}_{3}\left(1+t^{2}\right) \int_{-\widetilde{\beta}}^{\widetilde{\beta}}|\mu(\beta)|^{-3} d \beta+\varepsilon C_{1}\left(2 c_{b}\right)^{-1} t \int_{\widetilde{\beta}}^{\infty} \beta^{-2} d \beta\right) .
\end{aligned}
$$

(Recall that $\beta=\widetilde{\beta}$ (see (3.16) $)$ corresponds to the point $\widetilde{\zeta}_{t} \in \Pi_{t}$ with $\operatorname{Re} \widetilde{\zeta}_{t}=c_{b}+1$.) Note that $|\mu(\beta)| \geqslant \operatorname{Re} \mu(\beta)=c_{b}^{1 / 2}$. So, by (3.16),

$$
\int_{-\widetilde{\beta}}^{\widetilde{\beta}}|\mu(\beta)|^{-3} d \beta \leqslant 2 c_{b}^{-3 / 2} \widetilde{\beta}=2 c_{b}^{-3 / 2} \sqrt{c_{b}+t^{2}\left(c_{b}+1\right)} \leqslant \mathfrak{c}_{4}\left(t^{2}+1\right)^{1 / 2},
$$

where $\mathfrak{c}_{4}:=2 c_{b}^{-3 / 2}\left(c_{b}+1\right)^{1 / 2}$. Next, according to (3.16),

$$
\int_{\widetilde{\beta}}^{\infty} \beta^{-2} d \beta=\widetilde{\beta}^{-1}=\left(c_{b}+t^{2}\left(c_{b}+1\right)\right)^{-1 / 2} \leqslant t^{-1}\left(c_{b}+1\right)^{-1 / 2} .
$$

Bringing (3.20)-(3.22) together, we obtain

$$
\|\mathfrak{I}(\varepsilon ; t)\|_{L_{2}(\mathcal{O}) \rightarrow L_{2}(\mathcal{O})} \leqslant \mathfrak{c}_{5} \varepsilon t^{2}\left(\left(1+t^{2}\right)^{3 / 2}+1\right),
$$

where $\mathfrak{c}_{5}:=(2 \pi)^{-1} e^{\sqrt{c_{b}}} \max \left\{\mathfrak{c}_{3} \mathfrak{c}_{4} C_{2} ;\left(2 c_{b}\right)^{-1}\left(c_{b}+1\right)^{-1 / 2} C_{1}\right\}$.

Combining (3.3), (3.7), (3.8), and (3.23), we arrive at the estimate

$$
\left\|\left(\cos \left(t B_{D, \varepsilon}^{1 / 2}\right)-\cos \left(t\left(B_{D}^{0}\right)^{1 / 2}\right)\right)\left(B_{D}^{0}\right)^{-2}\right\|_{L_{2}(\mathcal{O}) \rightarrow L_{2}(\mathcal{O})} \leqslant \widehat{C}_{7} \varepsilon\left(1+t^{2}+t^{2}\left(1+t^{2}\right)^{3 / 2}\right)
$$

with the constant $\widehat{C}_{7}:=\max \left\{4 \mathcal{C}_{1} C_{16} ; 2^{-1} C_{16}+\mathfrak{c}_{5}\right\}$. Note that for $|t| \leqslant 1$ the leading degree of $t$ in the right-hand side of (3.24) is $t^{0}$, but for $|t|>1$ the leading degree is $t^{5}$. So, (3.24) implies the required estimate (2.5) with the constant $C_{7}:=2(1+\sqrt{2}) \widehat{C}_{7}$.

Combining the identity

$$
B_{D, \varepsilon}^{-1 / 2} \sin \left(t B_{D, \varepsilon}^{1 / 2}\right)=\int_{0}^{t} \cos \left(\tau B_{D, \varepsilon}^{1 / 2}\right) d \tau,
$$

the similar identity for the effective operator, and (2.5), we arrive at estimate (2.6) $)$. 


\subsection{Proof of Theorem 2.3 .}

Proof of Theorem 2.3. Without loss of generality, let $t>0$. Similarly to (3.5),

$$
\cos \left(t\left(B_{D}^{0}\right)^{1 / 2}\right)\left(B_{D}^{0}\right)^{-2}=-\frac{t^{2}}{2}\left(B_{D}^{0}\right)^{-1}+\left(B_{D}^{0}\right)^{-2}+\frac{1}{2 \pi i} \int_{\operatorname{Re} \lambda=\sqrt{c_{b}} / t} \lambda^{-3}\left(B_{D}^{0}+\lambda^{2} I\right)^{-1} e^{\lambda t} d \lambda .
$$

This implies that

$$
\begin{aligned}
\varepsilon\left(\Lambda^{\varepsilon} b(\mathbf{D})+\widetilde{\Lambda}^{\varepsilon}\right) S_{\varepsilon} P_{\mathcal{O}} \cos \left(t\left(B_{D}^{0}\right)^{1 / 2}\right)\left(B_{D}^{0}\right)^{-2} & =-\frac{\varepsilon t^{2}}{2} K_{D}(\varepsilon ; 0)+\varepsilon K_{D}(\varepsilon ; 0)\left(B_{D}^{0}\right)^{-1} \\
& +\frac{\varepsilon}{2 \pi i} \int_{\operatorname{Re} \lambda=\sqrt{c_{b}} / t} \lambda^{-3} K_{D}\left(\varepsilon ;-\lambda^{2}\right) e^{\lambda t} d \lambda .
\end{aligned}
$$

Here $K_{D}(\varepsilon ; \cdot)$ is the operator (1.58). Therefore, by (3.6)

$$
\begin{aligned}
& \cos \left(t B_{D, \varepsilon}^{1 / 2}\right) B_{D, \varepsilon}^{-2}-\cos \left(t\left(B_{D}^{0}\right)^{1 / 2}\right)\left(B_{D}^{0}\right)^{-2}-\varepsilon\left(\Lambda^{\varepsilon} b(\mathbf{D})+\widetilde{\Lambda}^{\varepsilon}\right) S_{\varepsilon} P_{\mathcal{O}} \cos \left(t\left(B_{D}^{0}\right)^{1 / 2}\right)\left(B_{D}^{0}\right)^{-2} \\
& =-\frac{t^{2}}{2}\left(B_{D, \varepsilon}^{-1}-\left(B_{D}^{0}\right)^{-1}-\varepsilon K_{D}(\varepsilon ; 0)\right)+\left(B_{D, \varepsilon}^{-2}-\left(B_{D}^{0}\right)^{-2}-\varepsilon K_{D}(\varepsilon ; 0)\left(B_{D}^{0}\right)^{-1}\right) \\
& +\frac{1}{2 \pi i} \int_{\operatorname{Re} \lambda=\sqrt{c_{b} / t}} \lambda^{-3}\left(\left(B_{D, \varepsilon}+\lambda^{2} I\right)^{-1}-\left(B_{D}^{0}+\lambda^{2} I\right)^{-1}-\varepsilon K_{D}\left(\varepsilon ;-\lambda^{2}\right)\right) e^{\lambda t} d \lambda .
\end{aligned}
$$

Denote the last summand in the right-hand side of $(3.26)$ by $\mathcal{I}(\varepsilon ; t)$.

Combining (1.20), (1.40), (1.54), and (1.60), we obtain

$$
\begin{aligned}
& \left\|B_{D, \varepsilon}^{-2}-\left(B_{D}^{0}\right)^{-2}-\varepsilon K_{D}(\varepsilon ; 0)\left(B_{D}^{0}\right)^{-1}\right\|_{L_{2}(\mathcal{O}) \rightarrow H^{1}(\mathcal{O})} \\
& \quad \leqslant\left\|B_{D, \varepsilon}^{-1}\left(B_{D, \varepsilon}^{-1}-\left(B_{D}^{0}\right)^{-1}\right)\right\|_{L_{2}(\mathcal{O}) \rightarrow H^{1}(\mathcal{O})} \\
& \quad+\left\|\left(B_{D, \varepsilon}^{-1}-\left(B_{D}^{0}\right)^{-1}-\varepsilon K_{D}(\varepsilon ; 0)\right)\left(B_{D}^{0}\right)^{-1}\right\|_{L_{2}(\mathcal{O}) \rightarrow H^{1}(\mathcal{O})} \\
& \quad \leqslant \mathcal{C}_{2} C_{2} \max \left\{1 ; c_{b}^{-2}\right\} \varepsilon+2 \max \left\{1 ; c_{b}^{-2}\right\} \mathcal{C}_{1} C_{4} \varepsilon^{1 / 2} \leqslant \mathfrak{c}_{6} \varepsilon^{1 / 2}
\end{aligned}
$$

where $\mathfrak{c}_{6}:=\max \left\{1 ; c_{\mathrm{b}}^{-2}\right\}\left(\mathcal{C}_{2} C_{2}+2 \mathcal{C}_{1} C_{4}\right)$.

By (1.60), (3.26), and (3.27),

$$
\begin{aligned}
& \| \cos \left(t B_{D, \varepsilon}^{1 / 2}\right)\left(B_{D, \varepsilon}\right)^{-2}-\cos \left(t\left(B_{D}^{0}\right)^{1 / 2}\right)\left(B_{D}^{0}\right)^{-2} \\
& \quad-\varepsilon\left(\Lambda^{\varepsilon} b(\mathbf{D})+\widetilde{\Lambda}^{\varepsilon}\right) S_{\varepsilon} P_{\mathcal{O}} \cos \left(t\left(B_{D}^{0}\right)^{1 / 2}\right)\left(B_{D}^{0}\right)^{-2} \|_{L_{2}(\mathcal{O}) \rightarrow H^{1}(\mathcal{O})} \\
& \quad \leqslant \max \left\{1 ; c_{b}^{-2}\right\} C_{4} \varepsilon^{1 / 2} t^{2}+\mathfrak{c}_{6} \varepsilon^{1 / 2}+\|\mathcal{I}(\varepsilon ; t)\|_{L_{2}(\mathcal{O}) \rightarrow H^{1}(\mathcal{O}) .}
\end{aligned}
$$

Changing the variable $\lambda t=\mu$ in the integral $\mathcal{I}(\varepsilon ; t)$, we get

$$
\mathcal{I}(\varepsilon ; t)=\frac{t^{2}}{2 \pi i} \int_{\operatorname{Re} \mu=\sqrt{c_{b}}} e^{\mu} \mu^{-3}\left(\left(B_{D, \varepsilon}+\frac{\mu^{2}}{t^{2}} I\right)^{-1}-\left(B_{D}^{0}+\frac{\mu^{2}}{t^{2}} I\right)^{-1}-\varepsilon K_{D}\left(\varepsilon ;-\frac{\mu^{2}}{t^{2}}\right)\right) d \mu .
$$

Let $\mu=\mu(\beta)=c_{b}^{1 / 2}+i \beta, \beta \in \mathbb{R}$. Then $\zeta_{t}(\beta)=-\mu(\beta)^{2} / t^{2}$ lies on the parabola $\Pi_{t}$ (see (3.10) ). Recall that $\widetilde{\beta}$ is defined by (3.16). For $-\widetilde{\beta} \leqslant \beta \leqslant \widetilde{\beta}$ we use estimate (1.60). By (3.11),

$$
\begin{aligned}
\varepsilon^{1 / 2} \varrho_{b}\left(\zeta_{t}\right)^{1 / 2}+\varepsilon\left|1+\zeta_{t}\right|^{1 / 2} \varrho_{b}\left(\zeta_{t}\right) & \leqslant \varepsilon^{1 / 2} \varrho_{b}\left(\zeta_{t}\right)\left(1+\left(1+\left|\zeta_{t}\right|\right)^{1 / 2}\right) \\
& \leqslant \varepsilon^{1 / 2} \varrho_{b}\left(\zeta_{t}\right)\left(1+\left(1+t^{-2}\left(c_{b}+\beta^{2}\right)\right)^{1 / 2}\right) .
\end{aligned}
$$

Together with (1.60) and (3.18), this implies

$$
\begin{aligned}
& \left\|\left(B_{D, \varepsilon}-\zeta_{t}(\beta) I\right)^{-1}-\left(B_{D}^{0}-\zeta_{t}(\beta) I\right)^{-1}-\varepsilon K_{D}\left(\varepsilon ; \zeta_{t}(\beta)\right)\right\|_{L_{2}(\mathcal{O}) \rightarrow H^{1}(\mathcal{O})} \\
& \leqslant \varepsilon^{1 / 2}\left(1+t^{2}\right) \mathfrak{c}_{3} C_{4}\left(2+t^{-1}\left(c_{\mathrm{b}}^{1 / 2}+\widetilde{\beta}\right)\right), \quad-\widetilde{\beta} \leqslant \beta \leqslant \widetilde{\beta} .
\end{aligned}
$$


For $|\beta|>\widetilde{\beta}$, we use (1.61), the estimate

$$
\begin{aligned}
|\mu(\beta)|^{-3} \frac{c\left(\phi_{t}\right)^{2}}{\left|\zeta_{t}(\beta)\right|^{1 / 4}} & =t^{-3}\left|\zeta_{t}(\beta)\right|^{-3 / 2} \frac{c\left(\phi_{t}\right)^{2}}{\left|\zeta_{t}(\beta)\right|^{1 / 4}}=t^{-3} \frac{\left|\zeta_{t}(\beta)\right|^{1 / 4}}{\left|\operatorname{Im} \zeta_{t}(\beta)\right|^{2}} \\
& =t^{-3} \frac{\left(t^{-2}\left(\beta^{2}+c_{b}\right)\right)^{1 / 4}}{t^{-4} 4 \beta^{2} c_{b}} \leqslant\left(4 c_{b}\right)^{-1} t^{1 / 2}\left(|\beta|^{-3 / 2}+c_{b}^{1 / 4} \beta^{-2}\right),
\end{aligned}
$$

and the identity

$$
c\left(\phi_{t}\right)^{3 / 2}|\mu(\beta)|^{-3}=\frac{1}{t^{3}\left|\operatorname{Im} \zeta_{t}(\beta)\right|^{3 / 2}}=2^{-3 / 2} c_{b}^{-3 / 4}|\beta|^{-3 / 2}
$$

We obtain that

$$
\begin{aligned}
& |\mu(\beta)|^{-3}\left\|\left(B_{D, \varepsilon}-\zeta_{t}(\beta) I\right)^{-1}-\left(B_{D}^{0}-\zeta_{t}(\beta) I\right)^{-1}-\varepsilon K_{D}\left(\varepsilon ; \zeta_{t}(\beta)\right)\right\|_{L_{2}(\mathcal{O}) \rightarrow H^{1}(\mathcal{O})} \\
& \quad \leqslant C_{5} \varepsilon^{1 / 2}\left(t^{1 / 2}\left(4 c_{b}\right)^{-1}\left(|\beta|^{-3 / 2}+c_{b}^{1 / 4} \beta^{-2}\right)+2^{-3 / 2} c_{b}^{-3 / 4}|\beta|^{-3 / 2}\right), \quad|\beta|>\widetilde{\beta} .
\end{aligned}
$$

From (3.29)-(3.31) and the estimate $|\mu(\beta)| \geqslant c_{b}^{1 / 2}$ it follows that

$$
\begin{aligned}
& \|\mathcal{I}(\varepsilon ; \zeta)\|_{L_{2}(\mathcal{O}) \rightarrow H^{1}(\mathcal{O})} \leqslant \frac{t^{2} e^{\sqrt{c_{b}}}}{2 \pi}\left(\mathfrak{c}_{3} C_{4}\left(1+t^{2}\right) \varepsilon^{1 / 2}\left(2+t^{-1}\left(c_{b}^{1 / 2}+\widetilde{\beta}\right)\right) \int_{-\widetilde{\beta}}^{\widetilde{\beta}}|\mu(\beta)|^{-3} d \beta\right. \\
& \left.\quad+2 \varepsilon^{1 / 2} t^{1 / 2}\left(4 c_{b}\right)^{-1} C_{5} \int_{\widetilde{\beta}}^{\infty}\left(\beta^{-3 / 2}+c_{b}^{1 / 4} \beta^{-2}\right) d \beta+2 \varepsilon^{1 / 2} C_{5} 2^{-3 / 2} c_{b}^{-3 / 4} \int_{\widetilde{\beta}}^{\infty} \beta^{-3 / 2} d \beta\right) \\
& \quad \leqslant \frac{t^{2} e^{\sqrt{c_{b}}}}{2 \pi}\left(\mathfrak{c}_{3} C_{4}\left(1+t^{2}\right) \varepsilon^{1 / 2} c_{b}^{-3 / 2}\left(2+t^{-1}\left(c_{b}^{1 / 2}+\widetilde{\beta}\right)\right) 2 \widetilde{\beta}+\varepsilon^{1 / 2} t^{1 / 2} c_{b}^{-1} C_{5} \widetilde{\beta}^{-1 / 2}\right. \\
& \left.\quad+\varepsilon^{1 / 2} t^{1 / 2}\left(2 c_{b}\right)^{-1} c_{b}^{1 / 4} C_{5} \widetilde{\beta}^{-1}+2^{1 / 2} \varepsilon^{1 / 2} C_{5} c_{b}^{-3 / 4} \widetilde{\beta}^{-1 / 2}\right) .
\end{aligned}
$$

By (3.16),$\left(1+t^{2}\right) \widetilde{\beta} \leqslant\left(1+t^{2}\right)^{3 / 2}\left(c_{b}+1\right)^{1 / 2}$ and $\widetilde{\beta}^{-1 / 2} \leqslant t^{-1 / 2}\left(c_{b}+1\right)^{-1 / 4}$. Then $t^{1 / 2} \widetilde{\beta}^{-1 / 2} \leqslant$ $\left(c_{b}+1\right)^{-1 / 4}$ and $t^{1 / 2} \widetilde{\beta}^{-1} \leqslant t^{-1 / 2}\left(c_{b}+1\right)^{-1 / 2}$. Therefore,

$$
\|\mathcal{I}(\varepsilon ; t)\|_{L_{2}(\mathcal{O}) \rightarrow H^{1}(\mathcal{O})} \leqslant \mathfrak{c}_{7} \varepsilon^{1 / 2} t^{2}\left(\left(1+t^{2}\right)^{3 / 2}\left(1+t^{-1}\right)+t^{-1 / 2}+1\right),
$$

where

$$
\begin{gathered}
\mathfrak{c}_{7}:=(2 \pi)^{-1} e^{\sqrt{c_{b}}} \max \left\{2 \mathfrak{c}_{3} C_{4} c_{\mathrm{b}}^{-3 / 2}\left(c_{b}+1\right)^{1 / 2} \max \left\{2+\left(c_{b}+1\right)^{1 / 2} ; 2 c_{b}^{1 / 2}\right\} ;\right. \\
\left.\left(c_{b}+1\right)^{-1 / 4} c_{b}^{-1} C_{5} ; 2^{-1} c_{b}^{-3 / 4}\left(c_{b}+1\right)^{-1 / 2} C_{5}+2^{1 / 2} C_{5} c_{b}^{-3 / 4}\left(c_{b}+1\right)^{-1 / 4}\right\} .
\end{gathered}
$$

Combining (3.28) and (3.32), we arrive at the estimate

$$
\begin{aligned}
& \| \cos \left(t B_{D, \varepsilon}^{1 / 2}\right) B_{D, \varepsilon}^{-2}-\cos \left(t\left(B_{D}^{0}\right)^{1 / 2}\right)\left(B_{D}^{0}\right)^{-2} \\
& \quad-\varepsilon\left(\Lambda^{\varepsilon} b(\mathbf{D})+\widetilde{\Lambda}^{\varepsilon}\right) S_{\varepsilon} P_{\mathcal{O}} \cos \left(t\left(B_{D}^{0}\right)^{1 / 2}\right)\left(B_{D}^{0}\right)^{-2} \|_{L_{2}(\mathcal{O}) \rightarrow H^{1}(\mathcal{O})} \\
& \quad \leqslant \widehat{C}_{10} \varepsilon^{1 / 2}\left(1+t^{2}\left(t^{-1 / 2}+1+\left(1+t^{2}\right)^{3 / 2}\left(1+t^{-1}\right)\right)\right) .
\end{aligned}
$$

Here $\widehat{C}_{10}:=\max \left\{C_{4} \max \left\{1 ; c_{b}^{-2}\right\}+\mathfrak{c}_{7} ; \mathfrak{c}_{6}\right\}$. Finally, by (1.18), (1.19), and (3.1),

$$
\begin{aligned}
& \left\|\cos \left(t B_{D, \varepsilon}^{1 / 2}\right) B_{D, \varepsilon}^{-1}\left(B_{D, \varepsilon}^{-1}-\left(B_{D}^{0}\right)^{-1}\right)\right\|_{L_{2}(\mathcal{O}) \rightarrow H^{1}(\mathcal{O})} \\
& \quad \leqslant c_{3}\left\|B_{D, \varepsilon}^{-1 / 2}\left(B_{D, \varepsilon}^{-1}-\left(B_{D}^{0}\right)^{-1}\right)\right\|_{L_{2}(\mathcal{O}) \rightarrow L_{2}(\mathcal{O})} \leqslant c_{3} \mathcal{C}_{1}^{1 / 2} C_{16} \varepsilon .
\end{aligned}
$$

Together with (3.33) this implies

$$
\begin{aligned}
& \|\left(\cos \left(t B_{D, \varepsilon}^{1 / 2}\right) B_{D, \varepsilon}^{-1}-\cos \left(t\left(B_{D}^{0}\right)^{1 / 2}\right)\left(B_{D}^{0}\right)^{-1}\right. \\
& \left.\quad-\varepsilon\left(\Lambda^{\varepsilon} b(\mathbf{D})+\widetilde{\Lambda}^{\varepsilon}\right) S_{\varepsilon} P_{\mathcal{O}} \cos \left(t\left(B_{D}^{0}\right)^{1 / 2}\right)\left(B_{D}^{0}\right)^{-1}\right)\left(B_{D}^{0}\right)^{-1} \|_{L_{2}(\mathcal{O}) \rightarrow H^{1}(\mathcal{O})} \\
& \quad \leqslant \widetilde{C}_{10} \varepsilon^{1 / 2}\left(1+t^{2}\left(1+t^{-1 / 2}+\left(1+t^{2}\right)^{3 / 2}\left(1+t^{-1}\right)\right)\right) .
\end{aligned}
$$


Here $\widetilde{C}_{10}:=\widehat{C}_{10}+c_{3} \mathcal{C}_{1}^{1 / 2} C_{16}$. Finally, note that for $|t|<1$ the leading degree of $t$ in the right-hand side of (3.34) is $t^{0}$, and for $|t| \geqslant 1$ the leading degree is $t^{5}$. Using this argument, from (3.34) we derive estimate (2.9) with $C_{10}:=\left(3+2^{5 / 2}\right) \widetilde{C}_{10}$.

\subsection{Proof of Theorem 2.2 .}

Proof of Theorem 2.2. By using Theorem 2.3, identity (3.25), and the similar identity for the effective operator, we obtain

$$
\begin{aligned}
\| & \left(B_{D, \varepsilon}^{-1 / 2} \sin \left(t B_{D, \varepsilon}^{1 / 2}\right) B_{D, \varepsilon}^{-1}-\left(B_{D}^{0}\right)^{-1 / 2} \sin \left(t\left(B_{D}^{0}\right)^{1 / 2}\right)\left(B_{D}^{0}\right)^{-1}\right. \\
& \left.-\varepsilon\left(\Lambda^{\varepsilon} b(\mathbf{D})+\widetilde{\Lambda}^{\varepsilon}\right) S_{\varepsilon} P_{\mathcal{O}}\left(B_{D}^{0}\right)^{-1 / 2} \sin \left(t\left(B_{D}^{0}\right)^{1 / 2}\right)\left(B_{D}^{0}\right)^{-1}\right)\left(B_{D}^{0}\right)^{-1} \|_{L_{2}(\mathcal{O}) \rightarrow H^{1}(\mathcal{O})} \\
& \leqslant C_{10} \varepsilon^{1 / 2}|t|\left(1+|t|^{5}\right), \quad t \in \mathbb{R}, \quad 0<\varepsilon \leqslant \varepsilon_{1} .
\end{aligned}
$$

Next, by (1.18), (1.40), and (3.1),

$$
\left\|B_{D, \varepsilon}^{-1 / 2} \sin \left(t B_{D, \varepsilon}^{1 / 2}\right)\left(B_{D, \varepsilon}^{-1}-\left(B_{D}^{0}\right)^{-1}\right)\left(B_{D}^{0}\right)^{-1}\right\|_{L_{2}(\mathcal{O}) \rightarrow H^{1}(\mathcal{O})} \leqslant c_{3} C_{16} \mathcal{C}_{1} \varepsilon .
$$

Combining (3.35) and (3.36), we arrive at estimate (2.7) with the constant

$$
C_{8}:=2\left(C_{10}+c_{3} C_{16} \mathcal{C}_{1}\right) \text {. }
$$

Let us check inequality (2.8). By (1.3) and (2.7), for $t \in \mathbb{R}$ and $0<\varepsilon \leqslant \varepsilon_{1}$ we have

$$
\begin{aligned}
& \|\left(g^{\varepsilon} b(\mathbf{D}) B_{D, \varepsilon}^{-1 / 2} \sin \left(t B_{D, \varepsilon}^{1 / 2}\right)\right. \\
& \left.\quad-g^{\varepsilon} b(\mathbf{D})\left(I+\varepsilon \Lambda^{\varepsilon} b(\mathbf{D}) S_{\varepsilon} P_{\mathcal{O}}+\varepsilon \widetilde{\Lambda}^{\varepsilon} S_{\varepsilon} P_{\mathcal{O}}\right)\left(B_{D}^{0}\right)^{-1 / 2} \sin \left(t\left(B_{D}^{0}\right)^{1 / 2}\right)\right) \\
& \quad \times\left(B_{D}^{0}\right)^{-2}\left\|_{L_{2}(\mathcal{O}) \rightarrow L_{2}(\mathcal{O})} \leqslant\left(d \alpha_{1}\right)^{1 / 2} C_{8}\right\| g \|_{L_{\infty}} \varepsilon^{1 / 2}\left(1+t^{6}\right) .
\end{aligned}
$$

Obviously,

$$
\begin{aligned}
& g^{\varepsilon} b(\mathbf{D})\left(I+\varepsilon \Lambda^{\varepsilon} b(\mathbf{D}) S_{\varepsilon} P_{\mathcal{O}}+\varepsilon \widetilde{\Lambda}^{\varepsilon} S_{\varepsilon} P_{\mathcal{O}}\right)\left(B_{D}^{0}\right)^{-1 / 2} \sin \left(t\left(B_{D}^{0}\right)^{1 / 2}\right)\left(B_{D}^{0}\right)^{-2} \\
& \quad=g^{\varepsilon} b(\mathbf{D})\left(B_{D}^{0}\right)^{-5 / 2} \sin \left(t\left(B_{D}^{0}\right)^{1 / 2}\right)+g^{\varepsilon}(b(\mathbf{D}) \Lambda)^{\varepsilon} S_{\varepsilon} b(\mathbf{D}) P_{\mathcal{O}}\left(B_{D}^{0}\right)^{-5 / 2} \sin \left(t\left(B_{D}^{0}\right)^{1 / 2}\right) \\
& \quad+g^{\varepsilon}(b(\mathbf{D}) \widetilde{\Lambda})^{\varepsilon} S_{\varepsilon} P_{\mathcal{O}}\left(B_{D}^{0}\right)^{-5 / 2} \sin \left(t\left(B_{D}^{0}\right)^{1 / 2}\right) \\
& \quad+\varepsilon \sum_{l=1}^{d} g^{\varepsilon} b_{l}\left(\Lambda^{\varepsilon} S_{\varepsilon} b(\mathbf{D}) D_{l}+\widetilde{\Lambda}^{\varepsilon} S_{\varepsilon} D_{l}\right) P_{\mathcal{O}}\left(B_{D}^{0}\right)^{-5 / 2} \sin \left(t\left(B_{D}^{0}\right)^{1 / 2}\right) .
\end{aligned}
$$

The fourth summand in the right-hand side of (3.38) can be estimated with the help of (1.3), (1.63) , and (1.64):

$$
\begin{aligned}
& \left\|\varepsilon \sum_{l=1}^{d} g^{\varepsilon} b_{l}\left(\Lambda^{\varepsilon} S_{\varepsilon} b(\mathbf{D}) D_{l}+\widetilde{\Lambda}^{\varepsilon} S_{\varepsilon} D_{l}\right) P_{\mathcal{O}}\left(B_{D}^{0}\right)^{-5 / 2} \sin \left(t\left(B_{D}^{0}\right)^{1 / 2}\right)\right\|_{L_{2}(\mathcal{O}) \rightarrow L_{2}\left(\mathbb{R}^{d}\right)} \\
& \quad \leqslant \varepsilon\left(d \alpha_{1}\right)^{1 / 2}\|g\|_{L_{\infty}} M_{1}\left\|b(\mathbf{D}) \mathbf{D} P_{\mathcal{O}}\left(B_{D}^{0}\right)^{-5 / 2} \sin \left(t\left(B_{D}^{0}\right)^{1 / 2}\right)\right\|_{L_{2}(\mathcal{O}) \rightarrow L_{2}\left(\mathbb{R}^{d}\right)} \\
& \quad+\varepsilon\left(d \alpha_{1}\right)^{1 / 2}\|g\|_{L_{\infty}} \widetilde{M}_{1}\left\|\mathbf{D} P_{\mathcal{O}}\left(B_{D}^{0}\right)^{-5 / 2} \sin \left(t\left(B_{D}^{0}\right)^{1 / 2}\right)\right\|_{L_{2}(\mathcal{O}) \rightarrow L_{2}\left(\mathbb{R}^{d}\right)} .
\end{aligned}
$$

Combining (1.2), (1.57), (2.15), and (3.39), we get

$$
\begin{aligned}
& \left\|\varepsilon \sum_{l=1}^{d} g^{\varepsilon} b_{l}\left(\Lambda^{\varepsilon} S_{\varepsilon} b(\mathbf{D}) D_{l}+\widetilde{\Lambda}^{\varepsilon} S_{\varepsilon} D_{l}\right) P_{\mathcal{O}}\left(B_{D}^{0}\right)^{-5 / 2} \sin \left(t\left(B_{D}^{0}\right)^{1 / 2}\right)\right\|_{L_{2}(\mathcal{O}) \rightarrow L_{2}\left(\mathbb{R}^{d}\right)} \\
& \quad \leqslant \varepsilon|t| \widehat{C}_{9}, \quad t \in \mathbb{R}, \quad 0<\varepsilon \leqslant 1,
\end{aligned}
$$

where $\widehat{C}_{9}:=\left(d \alpha_{1}\right)^{1 / 2}\|g\|_{L_{\infty}}\left(M_{1} \alpha_{1}^{1 / 2} C_{\mathcal{O}}^{(2)}+\widetilde{M}_{1} C_{\mathcal{O}}^{(1)}\right) \mathcal{C}_{1} \mathcal{C}_{3}$. 
By Proposition 1.1 and (1.2), (1.57), (2.15),

$$
\begin{aligned}
& \left\|g^{\varepsilon} b(\mathbf{D})\left(S_{\varepsilon}-I\right) P_{\mathcal{O}}\left(B_{D}^{0}\right)^{-5 / 2} \sin \left(t\left(B_{D}^{0}\right)^{1 / 2}\right)\right\|_{L_{2}(\mathcal{O}) \rightarrow L_{2}\left(\mathbb{R}^{d}\right)} \\
& \quad \leqslant \varepsilon r_{1}\|g\|_{L_{\infty}}\left\|\mathbf{D} b(\mathbf{D}) P_{\mathcal{O}}\left(B_{D}^{0}\right)^{-5 / 2} \sin \left(t\left(B_{D}^{0}\right)^{1 / 2}\right)\right\|_{L_{2}(\mathcal{O}) \rightarrow L_{2}\left(\mathbb{R}^{d}\right)} \\
& \quad \leqslant \varepsilon|t| r_{1}\|g\|_{L_{\infty}} \alpha_{1}^{1 / 2} C_{\mathcal{O}}^{(2)} \mathcal{C}_{1} \mathcal{C}_{3} .
\end{aligned}
$$

Combining (1.22), (3.37), (3.38), (3.40), and (3.41), we arrive at the required inequality (2.8) with the constant $C_{9}:=\left(d \alpha_{1}\right)^{1 / 2} C_{8}\|g\|_{L_{\infty}}+\widehat{C}_{9}+r_{1}\|g\|_{L_{\infty}} \alpha_{1}^{1 / 2} C_{\mathcal{O}}^{(2)} \mathcal{C}_{1} \mathcal{C}_{3}$.

\section{REFERENCES}

[ABriV] G. Allaire, M. Briane, and M. Vanninathan, A comparison between two-scale asymptotic expansions and Bloch wave expansions for the homogenization of periodic structures, SeMA Journal 73 (2016), no. 3, 237-259.

[BaPa] N. S. Bakhvalov and G. P. Panasenko, Homogenization: Averaging processes in periodic media. Mathematical problems in mechanics of composite materials, Math. Appl. (Soviet Ser.), vol. 36, Kluwer Acad. Publ. Group, Dordrecht, 1989.

[BeLPap] A. Bensoussan, J.-L. Lions, and G. Papanicolaou, Asymptotic analysis for periodic structures, corrected reprint of the 1978 original. AMS Chelsea Publishing, Providence, 2011.

[BSu1] M. Sh. Birman and T. A. Suslina, Second order periodic differential operators. Threshold properties and homogenization, Algebra i Analiz 15 (2003), no. 5, 1-108; English transl., St. Petersburg Math. J. 15 (2004), no. 5, 639-714.

[BSu2] M. Sh. Birman and T. A. Suslina, Homogenization with corrector term for periodic elliptic differential operators, Algebra i Analiz 17 (2005), no. 6, 1-104; English transl., St. Petersburg Math. J. 17 (2006), no. 6, 897-973.

[BSu3] M. Sh. Birman and T. A. Suslina, Homogenization with corrector term for periodic differential operators. Approximation of solutions in the Sobolev class $H^{1}\left(\mathbb{R}^{d}\right)$, Algebra i Analiz 18 (2006), no. 6, 1-130; English transl., St. Petersburg Math. J. 18 (2007), no. 6, 857-955.

[BSu4] M. Sh. Birman and T. A. Suslina, Operator error estimates in the homogenization problem for nonstationary periodic equations, Algebra i Analiz 20 (2008), no. 6, 30-107; English transl., St. Petersburg Math. J. 20 (2009), no. 6, 873-928.

[BrOtFMu] S. Brahim-Otsmane, G. A. Francfort, and F. Murat, Correctors for the homogenization of the wave and heat equations, J. Math. Pures Appl. 71 (1992), 197-231.

[BraLe] M. Brassart and M. Lenczner, A two scale model for the periodic homogenization of the wave equation, J. Math. Pures Appl. 93 (2010), no. 5, 474-517.

[CaDiCoCalMaMarG] J. Casado-Diaz, J. Couce-Calvo, F. Maestre, and J. D. Martin-Gomez, Homogenization and correctors for the wave equation with periodic coefficients, Math. Models Methods Appl. Sci. 24 (2014), 1343-1388.

[ChEl] R. Chill and A. F. M. ter Elst, Weak and strong approximation of semigroups on Hilbert spaces, Integr. Equ. Oper. Theory 90 (2018), no. 9.

[ConOrV] C. Conca, R. Orive, and M. Vanninathan, On Burnett coefficients in periodic media, J. Math. Phys. 47, 032902 (2006), no. 3.

[ConSaMaBalV] C. Conca, J. SanMartin, L. Balilescu, and M. Vanninathan, Optimal bounds on dispersion coefficient in one-dimensional periodic media, Math. Models Methods Appl. Sci. 19 (2009), no. 9, 1743-1764.

[CooSav] Sh. Cooper and A. Savostianov, Homogenisation with error estimates of attractors for damped semi-linear anisotropic wave equations, arXiv:1804.09947 (2018).

[DSu] M. A. Dorodnyi and T. A. Suslina, Spectral approach to homogenization of hyperbolic equations with periodic coefficients, J. Differential Equations 264 (2018), no. 12, 7463-7522.

[Gr1] G. Griso, Error estimate and unfolding for periodic homogenization, Asymptot. Anal. 40 (2004), no. $3 / 4,269-286$.

[Gr2] G. Griso, Interior error estimate for periodic homogenization, Anal. Appl. 4 (2006), no. 1, 61-79.

[GraRy] I. S. Gradshteyn and I. M. Ryzhik, Table of Integrals, Series, and Products, 7th Ed., ed. A. Jeffrey and D. Zwillinger, Academic Press, 2007.

[KeLiS] C. E. Kenig, F. Lin, and Z. Shen, Convergence rates in $L^{2}$ for elliptic homogenization problems, Arch. Rat. Mech. Anal. 203 (2012), no. 3, 1009-1036.

[KoE] V. A. Kondrat'ev and S. D. Eidel'man, About conditions on boundary surface in the theory of elliptic boundary value problems, Dokl. Akad. Nauk SSSR 246 (1979), no. 4, 812-815; English transl., Soviet Math. Dokl. 20 (1979), 261-263. 
[LaU] O. A. Ladyzhenskaya and N. N. Ural'tseva, Linear and quasilinear elliptic equations, Acad. Press, New York-London, 1968.

[MaSh] V. G. Maz'ya and T. O. Shaposhnikova, Theory of multipliers in spaces of differentiable functions, Monographs and Studies in Mathematica, vol. 23, Brookling (NY), 1985.

[McL] W. McLean, Strongly elliptic systems and boundary integral equations, Cambridge Univ. Press, Cambridge, 2000.

[M1] Yu. M. Meshkova, Homogenization of the Cauchy problem for parabolic systems with periodic coefficients, Algebra i Analiz 25 (2013), no. 6, 125-177; English transl., St. Petersburg Math. J. 25 (2014), no. 6, 981-1019.

[M2] Yu. Meshkova, On operator error estimates for homogenization of hyperbolic systems with periodic coefficients, arXiv:1705.02531 (2017).

[MSu1] Yu. M. Meshkova and T. A. Suslina, Two-parametric error estimates in homogenization of second order elliptic systems in $\mathbb{R}^{d}$, Appl. Anal. 95 (2016), no. 7, 1413-1448.

[MSu2] Yu. M. Meshkova and T. A. Suslina, Homogenization of initial boundary value problems for parabolic systems with periodic coefficients, Appl. Anal. 95 (2016), no. 8, 1736-1775.

[MSu3] Yu. M. Meshkova and T. A. Suslina, Homogenization of the Dirichlet problem for elliptic systems: Two-parametric error estimates, arXiv:1702.00550v4 (2017).

[MSu4] Yu. M. Meshkova, T. A. Suslina, Homogenization of the Dirichlet problem for elliptic and parabolic systems with periodic coefficients, Funktsional. Anal. i Prilozhen. 51 (2017), no. 3, 87-93; English transl.: Funct. Anal. Appl., 51 (2017), no. 3, 230-235.

[MSu5] Yu. M. Meshkova and T. A. Suslina, Homogenization of the first initial boundary-value problem for parabolic systems: operator error estimates, Algebra i Analiz 29 (2017), no. 6, 99-158; English transl.: St. Petersburg Math. J., 29 (2018), no. 6, to appear. See arXiv:1801.05035 (2018).

[Pas] S. E. Pastukhova, On the convergence of hyperbolic semigroups in variable Hilbert spaces, Trudy Seminara imeni I. G. Petrovskogo, 24 (2004), 216-241; English transl.: Journal of Mathematical Sciences 127 (2005), no. 5, 2263-2283.

[PSu] M. A. Pakhnin and T. A. Suslina, Operator error estimates for homogenization of the elliptic Dirichlet problem in a bounded domain, Algebra i Analiz 24 (2012), no. 6, 139-177; English transl., St. Petersburg Math. J. 24 (2013), no. 6, 949-976.

[R] V. S. Rychkov, On restrictions and extensions of the Besov and Triebel-Lizorkin spaces with respect to Lipschitz domains, J. London Math. Soc. 60 (1999), 237-257.

[Sa] E. Sanchez-Palencia, Non-homogeneous media and vibration theory, Lecture Notes in Physics, vol. 127, Springer-Verlag, Berlin, 1980.

[St] E. M. Stein, Singular integrals and differentiability properties of functions, Princeton University Press, Princeton, 1970.

[Su1] T. A. Suslina, On homogenization of periodic parabolic systems, Funktsional. Analiz i ego Prilozhen. 38 (2004), no. 4, 86-90; English transl., Funct. Anal. Appl. 38 (2004), no. 4, 309-312.

[Su2] T. A. Suslina, Homogenization of a periodic parabolic Cauchy problem in the Sobolev space $H^{1}\left(\mathbb{R}^{d}\right)$, Math. Model. Nat. Phenom. 5 (2010), no. 4, 390-447.

[Su3] T. A. Suslina, Homogenization in the Sobolev class $H^{1}\left(\mathbb{R}^{d}\right)$ for second order periodic elliptic operators with the inclusion of first order terms, Algebra i Analiz 22 (2010), no. 1, 108-222; English transl., St. Petersburg Math. J. 22 (2011), no. 1, 81-162.

[Su4] T. A. Suslina, Homogenization of the Dirichlet problem for elliptic systems: $L_{2}$-operator error estimates, Mathematika 59 (2013), no. 2, 463-476.

[Su5] T. A. Suslina, Homogenization of the Neumann problem for elliptic systems with periodic coefficients, SIAM J. Math. Anal. 45 (2013), no. 6, 3453-3493.

[ViGKo] N. Ya. Vilenkin, S. G. Krein et al., Functional Analysis, Groningen (Netherlands): WoltersNoordhoff Publishing, 1972.

$[\mathrm{Xu}] \quad \mathrm{Q} . \mathrm{Xu}$, Convergence rates for general elliptic homogenization problems in Lipschitz domains, SIAM J. Math. Anal. 48 (2016), no. 6, 3742-3788.

[ZhKO] V. V. Zhikov, S. M. Kozlov, and O. A. Oleঙnik, Homogenization of differential operators, SpringerVerlag, Berlin, 1994.

[Zh] V. V. Zhikov, On the operator estimates in the homogenization theory, Dokl. Ros. Akad. Nauk 403 (2005), no. 3, 305-308; English transl., Dokl. Math. 72 (2005), 535-538.

[ZhPas1] V. V. Zhikov and S. E. Pastukhova, On operator estimates for some problems in homogenization theory, Russ. J. Math. Phys. 12 (2005), no. 4, 515-524.

[ZhPas2] V. V. Zhikov and S. E. Pastukhova, Estimates of homogenization for a parabolic equation with periodic coefficients, Russ. J. Math. Phys. 13 (2006), no. 2, 224-237. 
[ZhPas3] V. V. Zhikov and S. E. Pastukhova, On the Trotter-Kato theorem in a variable space, Funktsional. Anal. i Prilozhen. 41 (2007), no. 4, 22-29; English transl., Funct. Anal. Appl. 41 (2007), no. 4, 264-270 .

[ZhPas4] V. V. Zhikov and S. E. Pastukhova, Operator estimates in homogenization theory, Uspekhi Matem. Nauk 71 (429) (2016), no. 3, 27-122; English transl., Russian Math. Surveys 71 (2016), no. 3, 417-511.

Chebyshev Laboratory, St. Petersburg State University, 14th Line V.O., 29B, St. PeTERsburg, 199178, Russia

E-mail address: y.meshkova@spbu.ru, juliavmeshke@yandex.ru 